Prepared in cooperation with the Bureau of Reclamation, the Colorado Water Conservation Board, and the Gunnison Basin Selenium Management Program

\title{
Groundwater Quality and Hydrology with Emphasis on Selenium Mobilization and Transport in the Lower Gunnison River Basin, Colorado, 2012-16
}

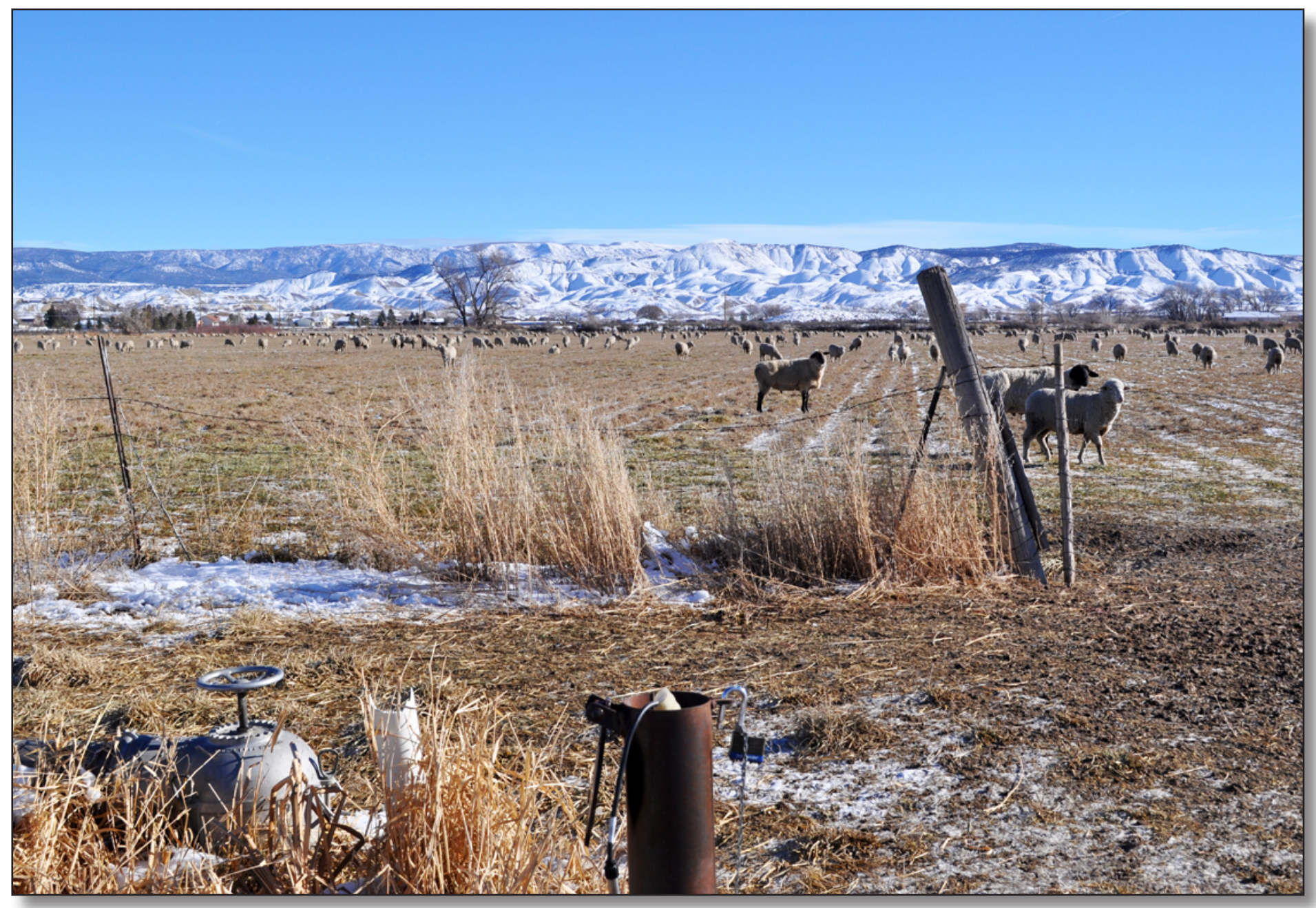

Scientific Investigations Report 2019-5029

U.S. Department of the Interior

U.S. Geological Survey 
Cover. Monitoring well 26, Montrose County, Colorado. Photograph by Judith Thomas, U.S. Geological Survey. 


\section{Groundwater Quality and Hydrology with Emphasis on Selenium Mobilization and Transport in the Lower Gunnison River Basin, Colorado, 2012-16}

By Judith C. Thomas, Peter B. McMahon, and L. Rick Arnold

Prepared in cooperation with the Bureau of Reclamation, the Colorado Water

Conservation Board, and the Gunnison Basin Selenium Management Program

Scientific Investigations Report 2019-5029 


\title{
U.S. Department of the Interior \\ DAVID BERNHARDT, Secretary
}

\author{
U.S. Geological Survey \\ James F. Reilly II, Director
}

U.S. Geological Survey, Reston, Virginia: 2019

For more information on the USGS - the Federal source for science about the Earth, its natural and living resources, natural hazards, and the environment-visit https://www.usgs.gov or call 1-888-ASK-USGS.

For an overview of USGS information products, including maps, imagery, and publications,

visit https://store.usgs.gov.

Any use of trade, firm, or product names is for descriptive purposes only and does not imply endorsement by the U.S. Government.

Although this information product, for the most part, is in the public domain, it also may contain copyrighted materials as noted in the text. Permission to reproduce copyrighted items must be secured from the copyright owner.

Suggested citation:

Thomas, J.C., McMahon, P.B., and Arnold, L.R., 2019, Groundwater quality and hydrology with emphasis on selenium mobilization and transport in the lower Gunnison River Basin, Colorado, 2012-16: U.S. Geological Survey Scientific Investigations Report 2019-5029, 69 p., https://doi.org/10.3133/sir20195029.

ISSN 2328-0328 (online) 


\section{Contents}

Abstract

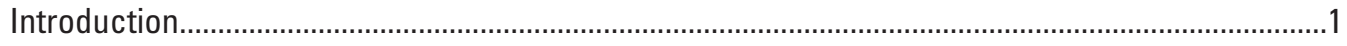

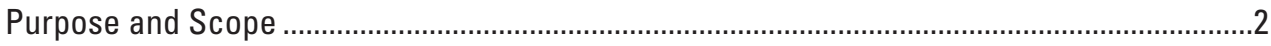

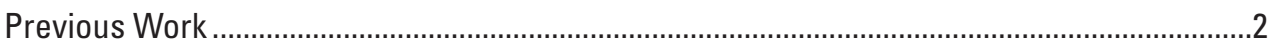

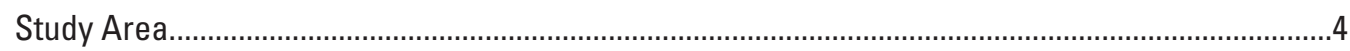

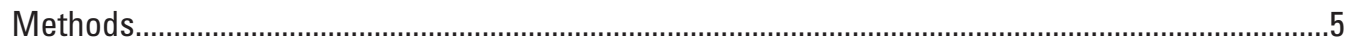

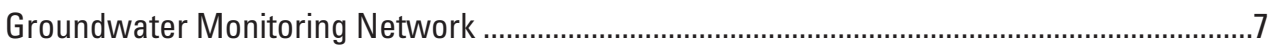

Water-Quality Sample Collection, Processing, and Analysis....................................................

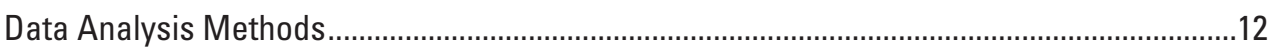

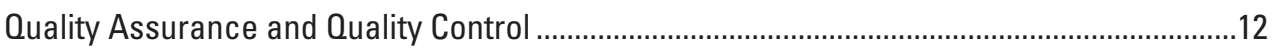

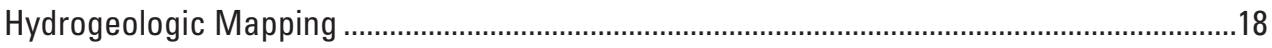

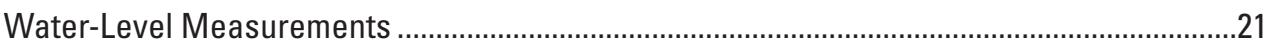

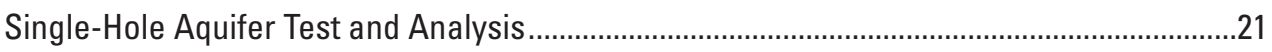

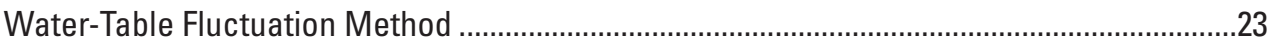

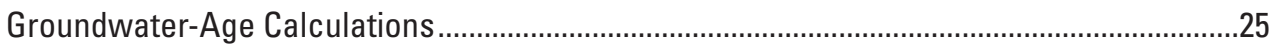

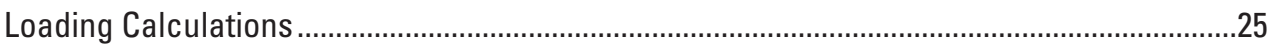

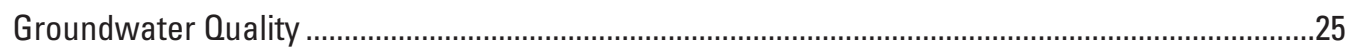

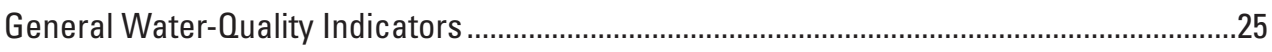

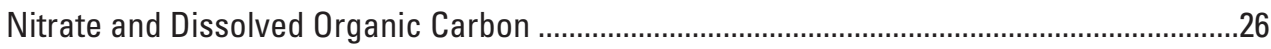

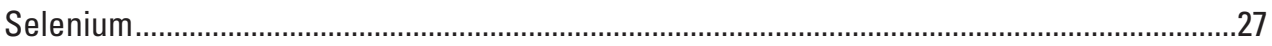

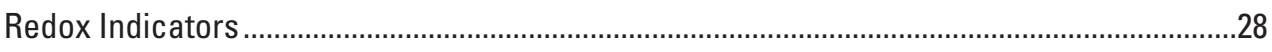

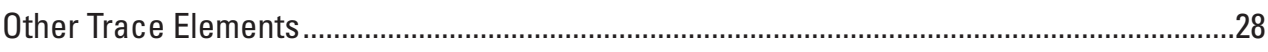

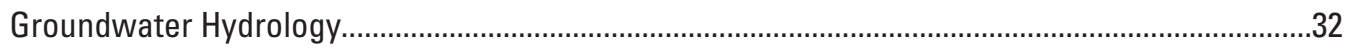

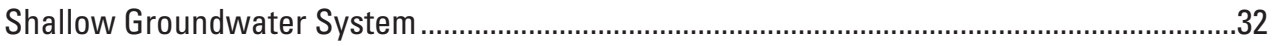

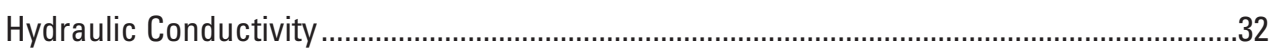

Water Level and Potentiometric Surface ...............................................................................32

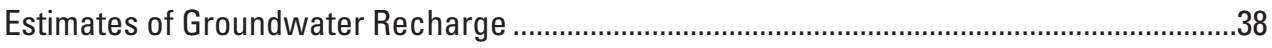

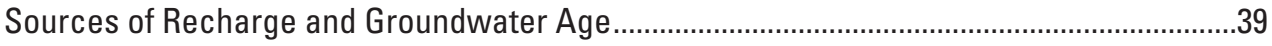

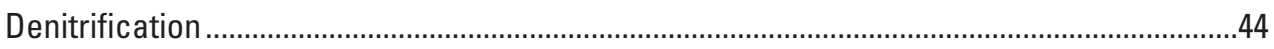

Estimates of Groundwater Discharge and Selenium Loading ..............................................4

Selenium Mobilization and Transport in Groundwater ................................................................46

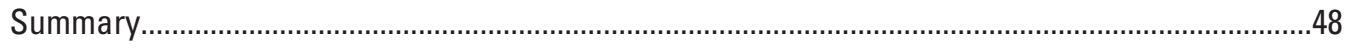

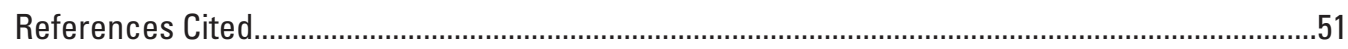

Appendix 1. Estimates of Recharge Using the Graphical Approach to the Water Table

Fluctuation (WTF) Method .................................................................................................56 


\section{Figures}

1. Regional map showing geographic features, generalized surficial geology, and location of the study area, east side of the Uncompahgre River, lower Gunnison River Basin, Colorado.

2. Generalized stratigraphic column for the lower Gunnison River Basin, Colorado............5

3. Land use of study area and location of monitoring wells, east side of the Uncompahgre River, lower Gunnison River Basin, Colorado ...............................................6

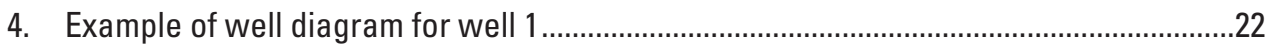

5. Example of slug-test trace for well 1 from spreadsheets developed by Halford and

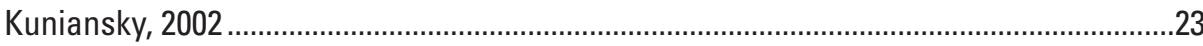

6. Example of slug-test analysis at well 1 from spreadsheets developed by Halford and Kuniansky, 2002

7. Water types grouped by the 25th, 50th, and 75th percentiles of specific conductance of groundwater, 2013-16.

8. Map showing mean selenium concentration at each monitoring well in the study area, 2013-16

9. Boxplots of selenium concentrations for $A$, the irrigation and nonirrigation seasons and $B$, weathered Mancos Shale and surficial deposits, 2013-16.

10. Boxplots of selenate concentration by reduction-oxidation (redox) group, 2013-16

11. Boxplots of boron, iron, manganese, lithium, strontium, and uranium concentration during the irrigation and nonirrigation seasons, 2013-16, with Colorado

Groundwater Agricultural Standards

12. Conceptual cross section of shallow groundwater system showing two scenarios of well completions

13. Thickness of surficial deposits and altitude of the consolidated bedrock surface, east side of the Uncompahgre River, Colorado

14. Estimated altitude of the potentiometric contours, based on water levels measured during 2012-16; areas where the shallow groundwater system is expected to be unsaturated or thin, discontinuous, or transient; and estimated areas where the shallow groundwater system is expected to be saturated, east side of the Uncompahgre River, Colorado.

15. Seasonal change in water levels in monitoring wells, 2014-16...................................38

16. Depths to water measurements for wells 4 and 25 for April 2014-November 2016 ........39

17. Stable isotopic composition of groundwater and surface water from the east side of the Uncompahgre River, lower Gunnison River Basin, Colorado, 2014-15, and local and global meteoric water lines

18. Data on dissolved-gas concentrations (dinitrogen and argon) in groundwater collected from monitoring wells during the irrigation and nonirrigation seasons, 2014-15, east side of the Uncompahgre River, lower Gunnison River Basin, Colorado.

19. Concentrations of tritium, ${ }^{3} \mathrm{He}_{\text {trit }}$ (tritiogenic helium-3), and $\mathrm{SF}_{6}$ (sulfur hexafluoride) in groundwater, 2014-15, and model results from TracerLPM

20. Tritium concentrations in samples collected in 2015 as a function of recharge year for the samples that were age dated or as a function of sample year for samples that were not age dated, and model results from TracerLPM

21. Isotopic fractionation of nitrate undergoing denitrification, assuming various starting nitrate concentrations and fractionation factors 
22. Conceptual model of selenium mobilization and transport in the shallow groundwater system on the east side of the Uncompahgre River, lower Gunnison River Basin, Colorado.

1.1. Peak water-table rise values (in red) used to determine estimated recharge for well 2 based on the graphical approach to the water-table fluctuation method .56

1.2. Peak water-table rise values (in red) used to determine estimated recharge for well 3 based on the graphical approach to the water-table fluctuation method

1.3. Peak water-table rise values (in red) used to determine estimated recharge for well 6 based on the graphical approach to the water-table fluctuation method . .58

1.4. Peak water-table rise values (in red) used to determine estimated recharge for well 7 based on the graphical approach to the water-table fluctuation method

1.5. Peak water-table rise values (in red) used to determine estimated recharge for well 11 based on the graphical approach to the water-table fluctuation method

1.6. Peak water-table rise values (in red) used to determine estimated recharge for well 12 based on the graphical approach to the water-table fluctuation method ..........61

1.7. Peak water-table rise values (in red) used to determine estimated recharge for well 13 based on the graphical approach to the water-table fluctuation method

1.8. Peak water-table rise values (in red) used to determine estimated recharge for well 15 based on the graphical approach to the water-table fluctuation method

1.9. Peak water-table rise values (in red) used to determine estimated recharge for well 18 based on the graphical approach to the water-table fluctuation method .64

1.10. Peak water-table rise values (in red) used to determine estimated recharge for well 20 based on the graphical approach to the water-table fluctuation method

1.11. Peak water-table rise values (in red) used to determine estimated recharge for well 22 based on the graphical approach to the water-table fluctuation method

1.12. Peak water-table rise values (in red) used to determine estimated recharge for well 23 based on the graphical approach to the water-table fluctuation method ..........67

1.13. Peak water-table rise values (in red) used to determine estimated recharge for well 24 based on the graphical approach to the water-table fluctuation method

1.14. Peak water-table rise values (in red) used to determine estimated recharge for well 29 based on the graphical approach to the water-table fluctuation method

\section{Tables}

1. Location, construction, and geologic information for monitoring wells, east side of the Uncompahgre River, lower Gunnison River Basin, Colorado

2. Summary of sampling events and analysis done at monitoring wells, east side of the Uncompahgre River, lower Gunnison River Basin, Colorado, 2013-16.

3. Constituents, result units, reporting limit, and analyzing laboratories for water-quality samples.

4. Results of sample-blank analyses for study period

5. Results of sample-replicate analyses for study period

6. Selenium, selenate, and selenite concentration from the National Water Quality Lab (NWQL) and the U.S. Geological Survey Colorado Water Science Center Water-Quality Research Lab (USGSCORL) and the calculated relative percent difference for NWOL and USGSCORL selenium results 
7. Relative percent difference for the National Water Quality Laboratory (NWOL) and the U.S. Geological Survey Colorado Water Science Center Water-Quality Research Lab (USGSCORL) analysis results and National Institute of Standards and Technology (NIST) selenium standard...

8. Summary statistics for select field properties, major ions, and nutrients for water collected from the monitoring wells, east side of the Uncompahgre River, lower Gunnison River Basin, Colorado, 2013-16

9. Summary statistics for select trace elements for water collected from the monitoring wells, east side of the Uncompahgre River, lower Gunnison River Basin, Colorado, 2013-16.

10. Estimated hydraulic-conductivity values for monitoring wells, east side of the Uncompahgre River, lower Gunnison River Basin, Colorado.

11. Summary of monthly manual water level measurements including period of record, number of measurements, and minimum and maximum observed water levels for monitoring wells, east side of the Uncompahgre River, lower Gunnison River Basin, Colorado

12. Estimated recharge based on the water table fluctuation (WTF) method for unconfined wells, Delta and Montrose Counties, Colorado

13. Tritium data for water collected from monitoring wells during the nonirrigation season in 2015, east side of the Uncompahgre River, lower Gunnison River Basin, Colorado

14. Estimates of groundwater age for select monitoring wells, east side of the Uncompahgre River, lower Gunnison River Basin, Colorado.

15. Summary of selenium load at Sunflower Drain at Highway 92 (USGS ID 384551107591901) compared to estimated base-flow contribution, 1991-2016

16. Summary of selenium load at Loutsenhizer Arroyo below North River Road near Delta, Colo., (USGS, ID 383946107595301) compared to estimated base-flow contribution, 1991-2016 


\section{Conversion Factors}

U.S. customary units to International System of Units

\begin{tabular}{|c|c|c|}
\hline Multiply & By & To obtain \\
\hline \multicolumn{3}{|c|}{ Length } \\
\hline inch (in.) & 2.54 & centimeter $(\mathrm{cm})$ \\
\hline inch (in.) & 25.4 & millimeter (mm) \\
\hline foot $(\mathrm{ft})$ & 0.3048 & meter $(\mathrm{m})$ \\
\hline mile (mi) & 1.609 & kilometer (km) \\
\hline \multicolumn{3}{|c|}{ Area } \\
\hline acre & 4,047 & square meter $\left(\mathrm{m}^{2}\right)$ \\
\hline acre & 0.4047 & hectare (ha) \\
\hline acre & 0.4047 & square hectometer $\left(\mathrm{hm}^{2}\right)$ \\
\hline acre & 0.004047 & square kilometer $\left(\mathrm{km}^{2}\right)$ \\
\hline square mile $\left(\mathrm{mi}^{2}\right)$ & 259.0 & hectare (ha) \\
\hline square mile $\left(\mathrm{mi}^{2}\right)$ & 2.590 & square kilometer $\left(\mathrm{km}^{2}\right)$ \\
\hline \multicolumn{3}{|c|}{ Volume } \\
\hline gallon (gal) & 3.785 & liter (L) \\
\hline gallon (gal) & 0.003785 & cubic meter $\left(\mathrm{m}^{3}\right)$ \\
\hline gallon (gal) & 3.785 & cubic decimeter $\left(\mathrm{dm}^{3}\right)$ \\
\hline cubic foot $\left(\mathrm{ft}^{3}\right)$ & 28.32 & cubic decimeter $\left(\mathrm{dm}^{3}\right)$ \\
\hline cubic foot $\left(\mathrm{ft}^{3}\right)$ & 0.02832 & cubic meter $\left(\mathrm{m}^{3}\right)$ \\
\hline \multicolumn{3}{|c|}{ Flow rate } \\
\hline foot per second (ft/s) & 0.3048 & meter per second $(\mathrm{m} / \mathrm{s})$ \\
\hline cubic foot per second $\left(\mathrm{ft}^{3} / \mathrm{s}\right)$ & 0.02832 & cubic meter per second $\left(\mathrm{m}^{3} / \mathrm{s}\right)$ \\
\hline gallon per minute (gal/min) & 0.06309 & liter per second $(\mathrm{L} / \mathrm{s})$ \\
\hline \multicolumn{3}{|c|}{ Hydraulic conductivity } \\
\hline foot per day (ft/d) & 0.3048 & meter per day $(\mathrm{m} / \mathrm{d})$ \\
\hline \multicolumn{3}{|c|}{ Hydraulic gradient } \\
\hline foot per mile (ft/mi) & 0.1894 & meter per kilometer $(\mathrm{m} / \mathrm{km})$ \\
\hline
\end{tabular}

Temperature in degrees Celsius $\left({ }^{\circ} \mathrm{C}\right)$ may be converted to degrees Fahrenheit $\left({ }^{\circ} \mathrm{F}\right)$ as follows:

$$
{ }^{\circ} \mathrm{F}=\left(1.8 \times{ }^{\circ} \mathrm{C}\right)+32 \text {. }
$$




\section{Datum}

Vertical coordinate information is referenced to the North American Vertical Datum of 1988 (NAVD 88).

Horizontal coordinate information is referenced to the North American Datum of 1983 (NAD 83).

Altitude, as used in this report, refers to distance above the vertical datum.

\section{Supplemental Information}

Specific conductance is given in microsiemens per centimeter at 25 degrees Celsius $(\mu \mathrm{S} / \mathrm{cm}$ at $\left.25^{\circ} \mathrm{C}\right)$.

Concentrations of chemical constituents in water are given in either milligrams per liter (mg/L) or micrograms per liter $(\mu \mathrm{g} / \mathrm{L})$. 


\title{
Groundwater Quality and Hydrology with Emphasis on Selenium Mobilization and Transport in the Lower Gunnison River Basin, Colorado, 2012-16
}

\author{
By Judith C. Thomas, Peter B. McMahon, and L. Rick Arnold
}

\section{Abstract}

Dissolved selenium is a contaminant of concern in the lower Gunnison River Basin, Colorado. Selenium is naturally present in the Cretaceous Mancos Shale and is leached to groundwater and surface water by irrigation. The groundwater on the east side of the Uncompahgre River in Delta and Montrose Counties is one of the primary sources of selenium concentration and load to surface water in the lower Gunnison River Basin. Although little information about the contribution of groundwater to surface water has been historically available, groundwater has often been implicated as an appreciable source of selenium to surface water. From 2012 to 2016, the U.S. Geological Survey, in cooperation with the Bureau of Reclamation, the Colorado Water Conservation Board, and the Gunnison Basin Selenium Management Program, established a 30-well groundwater-monitoring network on irrigated land to characterize the hydrology and groundwater quality of the shallow groundwater system on the east side of the Uncompahgre River in the lower Gunnison River Basin. The installation of the 30-well network and the data collected allowed for the development of a conceptual model of selenium mobilization and transport in the shallow groundwater system. Monitoring wells were completed in surficial deposits and in weathered Mancos Shale, which generally exhibited unconfined and confined conditions, respectively. Groundwater-quality monitoring provides information on the distribution of selenium and the geochemical processes controlling selenium concentrations in shallow groundwater. Monitoring wells were sampled between August 2013 and March 2015 to understand groundwater quality, seasonality, sources of recharge, and groundwater age. Concentrations of dissolved selenium ranged from below the limit of detection to 4,100 micrograms per liter $(\mu \mathrm{g} / \mathrm{L})$, with a median concentration of $14 \mu \mathrm{g} / \mathrm{L}$. Concentrations showed a high degree of spatial variability and no seasonal difference. Similarly, no seasonal pattern was observed in specific conductance values of groundwater despite the considerably lower specific conductance value of irrigation water.

Reduction-oxidation processes are important controls on selenium mobility. Nitrate derived from geologic material was a primary control on reduction-oxidation conditions in groundwater and inhibited selenium reduction to less mobile forms. Nitrate was reduced by denitrification in groundwater, but it was not reduced to the extent necessary to allow for selenium reduction. Groundwater ages were determined for groundwater samples from eight wells and ranged from 6 to 20 years old. Isotopic data indicate groundwater was recharged by irrigation water; no information collected supported an older, deeper source of recharge to the shallow groundwater system. Data on water level in all wells showed response to irrigation practices, but the response was delayed in some wells, which may be an indication of distance from recharge source.

\section{Introduction}

Dissolved selenium is a contaminant of concern in the lower Gunnison River Basin in Colorado. The Colorado Department of Public Health and Environment (CDPHE) has established dissolved selenium standards (CDPHE, 2016), including the chronic aquatic-life standard (4.6 micrograms per liter $[\mu \mathrm{g} / \mathrm{L}])$ and the acute aquatic-life standard $(18.4 \mu \mathrm{g} / \mathrm{L})$, and the U.S. Environmental Protection Agency (EPA; 2017) has established a drinking-water standard $(50 \mu \mathrm{g} / \mathrm{L})$. Many rivers and streams in the lower Gunnison River Basin are designated as selenium impaired according to the CDPHE chronic aquatic-life standard (CDPHE, 2016).

The process of reduction-oxidation (redox) is the transfer of electrons from one chemical species to another (Langmuir, 1997). In the environment, selenium has four oxidation states (-II, 0, IV, and VI), which can all occur together in soils (McNeal and Balistrieri, 1989). The most mobile form of selenium in the aqueous system is selenate (VI), but selenate can become immobile by reduction to elemental selenium (0), formation of metal selenides (-II), or selenite (IV) absorption (McNeal and Balistrieri, 1989). The biogeochemistry of selenium and environmental parameters $(\mathrm{pH}$ and redox conditions) influence the concentration, mobility, and distribution of selenium in the environment (McNeal and Balistrieri, 1989). Redox processes in particular are an important control on selenium in which the presence of higher redox species (oxygen and nitrate) prevents the reduction of mobile selenate to less mobile selenite (White and others, 1991). 
Historically, selenium concentrations in groundwater were not well characterized in the lower Gunnison River Basin; rather, the focus of monitoring has been on surface water. Although little is known about the contribution of groundwater to surface water, groundwater has often been implicated as a source of selenium to surface water (Butler and others, 1996; Butler and Leib, 2002; Mast and others, 2014; Linard and others, 2016; Mills and others, 2016). Selenium is naturally present in the Cretaceous Mancos Shale and is leached to groundwater and surface water by irrigation (Butler and others, 1996). On a regional scale, the Mancos Shale is not water bearing and is considered a confining unit because of its thickness and low permeability (Lohman, 1965). On a local scale, wells have been completed in the weathered Mancos Shale, typically at shallow depths (Lohman, 1965; Butler and others, 1996). Irrigation projects on the east side of the Uncompahgre River convey water that originates as snowmelt from higher elevation areas to lower elevation areas.

Widespread irrigation and the presence of the Mancos Shale combine to make the east side of the Uncompahgre River (fig. 1) one of the primary areas in the lower Gunnison River Basin where high selenium concentrations have been observed (Bureau of Reclamation, 2011). Selenium concentrations were measured from surface water during winter base flow; resulting data indicate that shallow groundwater is enriched with respect to selenium and demonstrate the need to develop a better understanding of the hydrology and groundwater quality of the shallow groundwater system in the lower Gunnison River Basin. The U.S. Geological Survey (USGS), in cooperation with the Bureau of Reclamation (Reclamation), the Colorado Water Conservation Board, and the Gunnison Basin Selenium Management Program, established a 30-well groundwater-monitoring network on irrigated land on the east side of the Uncompahgre River in the lower Gunnison River Basin (fig. 1) to characterize the hydrology and groundwater quality of the shallow groundwater system. Groundwater-quality monitoring provided information on the distribution of selenium and the geochemical processes controlling selenium concentrations in the shallow groundwater system. Groundwater hydrology was characterized by using water levels, potentiometric surface maps, aquifer characteristics, and estimates of groundwater recharge and discharge; this information is needed in order to better understand interactions between groundwater and surface water.

\section{Purpose and Scope}

The purpose of this report is to characterize the groundwater quality and hydrology of the shallow groundwater system on the east side of the Uncompahgre River in the lower Gunnison River Basin. Beginning in 2012, a 30-well groundwater-monitoring network was established on irrigated land underlain by Mancos Shale. Groundwater quality was monitored during 2013, 2014, and 2015 at the 30 monitoring wells to provide information on the distribution of selenium and on geochemical processes controlling selenium concentrations in the shallow groundwater system. Water-level data (collected from 2012 to 2016), potentiometric surface maps, aquifer-test results, and estimates of groundwater recharge and discharge were used to characterize groundwater hydrology.

\section{Previous Work}

Although numerous water-quality studies have been conducted, few have focused on selenium in groundwater and the contributions that groundwater makes to selenium in surface water in the lower Gunnison River Basin. Perennial streamflow in this basin is supported by seasonal tributary inflow, irrigationreturn flows, and shallow groundwater discharge from areas underlain by selenium-bearing shale. Previous work in the basin supports a conceptual model of water-table (unconfined) groundwater conditions present beneath irrigated areas and groundwater as an appreciable contributor of salt load to streams (Reclamation, 1982). A study by Warner and others (1985) on groundwater and salinity in the Upper Colorado River Basin indicated that groundwater contributes anywhere from 30 to 93 percent of the salt load in the Upper Colorado River Basin. Warner and others (1985) also estimated that the Uncompahgre River Basin produced the majority of the salt load in the lower Gunnison River Basin (45 percent). These studies were primarily focused on salinity and indicated that groundwater was a substantial contributor of salinity to surface water in western Colorado.

In October 1985, the National Irrigation Water Quality Program was created by the Department of Interior to improve understanding of the existence, magnitude, and causes of irrigation-induced contamination (Butler and others, 1991). This program resulted in several studies conducted in the lower Gunnison River Basin. A study by Butler and others (1991) investigated water quality, sediment, and biota associated with irrigation drainage in the Gunnison and Uncompahgre River Basins and Sweitzer Lake and characterized groundwater quality in the alluvium, Dakota Sandstone, and Mancos Shale in the Uncompahgre River Basin. Selenium concentrations in 15 groundwater samples ( 8 samples from the Mancos Shale) ranged from below the detection limit to $100 \mu \mathrm{g} / \mathrm{L}$, with a median of $8 \mu \mathrm{g} / \mathrm{L}$. The study identified surface water in the Uncompahgre River Basin as being enriched with respect to selenium for those cases in which the highest selenium concentrations were measured during base-flow conditions for the Uncompahgre River (November and January) (Butler and others, 1991). Building on the results of this study, the USGS conducted further investigations of selenium in water, sediment, and biota in the Uncompahgre River Basin and the Grand Valley, where additional groundwater-quality data were collected from wells completed in the Mancos Shale residuum and alluvium (Butler and others, 1996). A total of six wells were used to characterize selenium concentrations in groundwater near the Uncompahgre River and in the Grand Valley; selenium concentrations were highly variable between wells. Wells located on the east side of the Uncompahgre River had selenium concentrations ranging from 45 to $60 \mu \mathrm{g} / \mathrm{L}$, and wells completed in the alluvium in the Grand Valley had selenium concentrations over $1,000 \mu \mathrm{g} / \mathrm{L}$. 


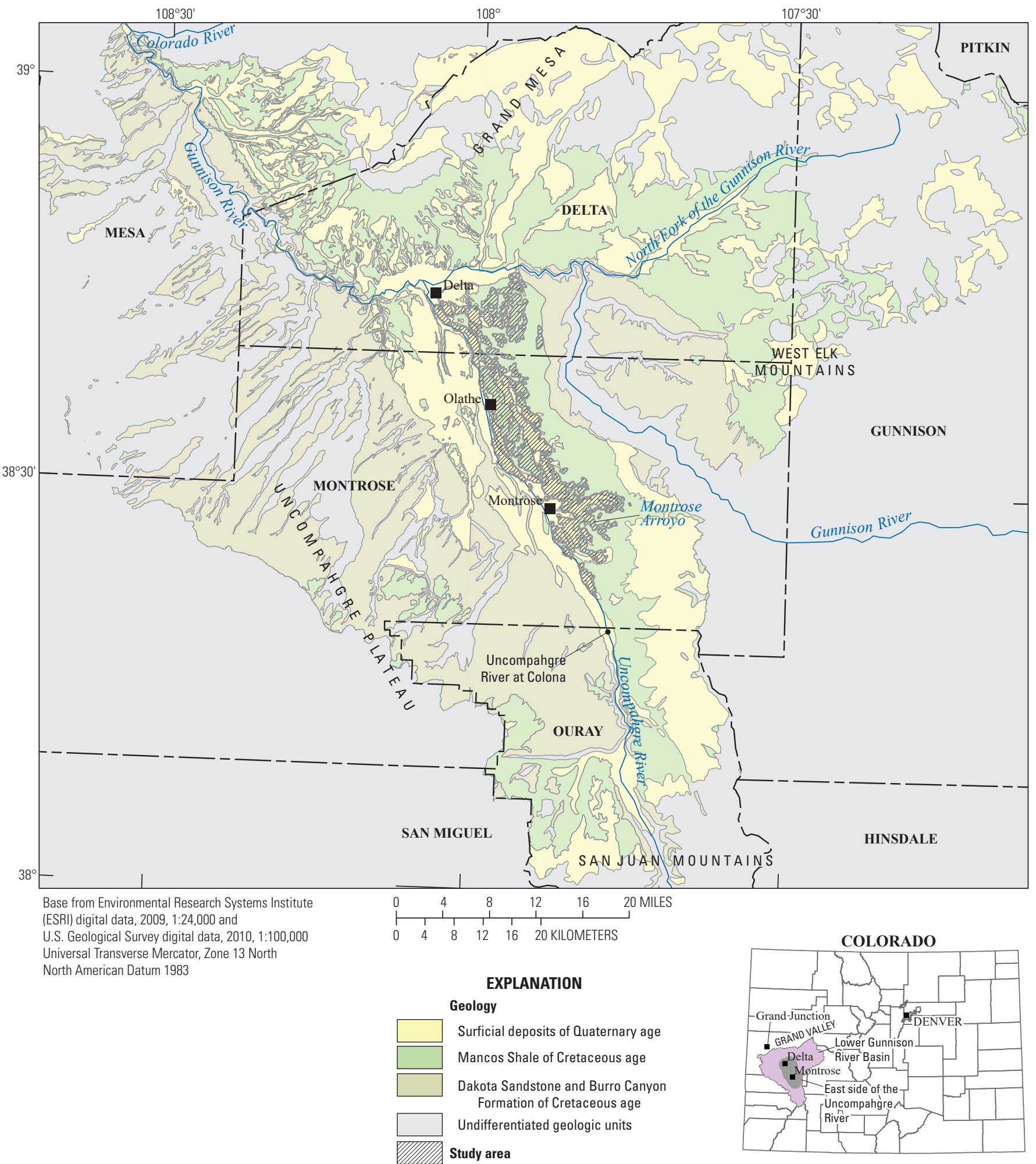

Figure 1. Geographic features, generalized surficial geology, and location of the study area, east side of the Uncompahgre River, lower Gunnison River Basin, Colorado. Geology modified from Tweto (1979). 
As knowledge of irrigation-induced contamination improved, studies began to focus on understanding how changing land use could result in changes to irrigationinduced contamination. In a USGS study in 2008, changes in land use were investigated with respect to the amount of irrigation water that recharges shallow groundwater through deep percolation (Mayo, 2008). The results of this study indicated that the conversion from agricultural land use to urban or residential land use substantially decreased the amount of recharge to shallow groundwater, the result of a substantial decrease in the amount of irrigation water applied. The study did not refer directly to shallow groundwater, but it concluded that this decrease in recharge had the potential to decrease salinity loading to surface water. This study was conducted in the Grand Valley, and percolation was measured at relatively shallow depths (1 foot [ft]), but its results have important implications to mechanisms of recharge to shallow groundwater systems in irrigated areas.

Improvements in irrigation efficiency result in less water applied to the land. One aspect of improved irrigation efficiency is improved delivery of irrigation water, such as piping and lining of canals reducing leakage. Butler (2001) conducted a pilot project in Montrose Arroyo, located on the south side of the City of Montrose, Colo., to understand how piping of canals would affect surface-water quality. Initial results indicated that piping canals improved surface-water quality. During continued monitoring in Montrose Arroyo, an increase in selenium loads and concentrations was observed from 1992 to 2010; the source of the selenium was hypothesized to be groundwater (Moore, 2011). Since 2010, selenium loads and concentrations have declined in Montrose Arroyo, and the USGS currently hypothesizes (Richards and Moore, 2015) that land disturbance related to development and construction was responsible for the increases observed prior to 2010.

Because selenium is a contaminant of concern in Colorado's streams and rivers, researchers continue to study waterquality issues related to selenium to further understand the role of geology and the mechanisms of selenium mobilization. A study by Paschke and others (2012) focused on geologic processes affecting selenium loading in Tollgate Creek, Aurora, Colo. The study found that selenium was mobilized by increased water use associated with urbanization, which resulted in increased recharge and discharge of groundwater. The conceptual model developed from this study identified processes mobilizing selenium. A subsequent study (Paschke and others, 2014) presented information on geologic sources and geochemical processes controlling selenium concentrations in the aquatic environment. Several geologic sources of selenium were found, including salts formed from secondary weathering processes.

More recent studies have investigated selenium in groundwater and in geologic material in the lower Gunnison River Basin by focusing on understanding the mechanisms that mobilize selenium. Changes in selenium concentration in groundwater were evaluated by Linard and others (2016) in a wetland located in the lower Gunnison River Basin. The wetland was formed by leakage from a nearby canal. Nested wells were installed to try to understand changes in selenium concentration in response to canal leakage. The high concentrations of selenium, nitrate, and dissolved organic carbon observed in groundwater were sourced from the Mancos Shale and its weathering products. Mast and others (2014) evaluated source materials by using sequential extractions from soils in irrigated and nonirrigated areas. In nonirrigated areas, most selenium was found in soluble sodium salts, whereas irrigated soils were depleted of soluble selenium. The study concluded that high concentrations of extractable nitrate found in soils and bedrock are important to selenium mobility because nitrate inhibits selenium reduction. Mills and others (2016) analyzed groundwater-quality data and solid-phase geochemical data from the lower Gunnison River Basin to understand controls on selenium distribution and mobilization in shallow groundwater systems. They showed that soluble salts are the primary source of selenium to the shallow groundwater system but constitute a small percentage of the total selenium content of solid-phase material. Oxidation of reduced selenium species, which constitute the majority of the selenium pool in the study area, could be a potential source of selenium in the future as soluble salts are progressively depleted.

\section{Study Area}

The study area is located in southwest Colorado and is bounded on the north by the Gunnison River and on the west by the Uncompahgre River (fig. 1). Geographically, the study area is bounded to the north by the Grand Mesa, to the east by the West Elk Mountains, to the south by the San Juan Mountains, and to the west by the Uncompahgre Plateau. The Gunnison River flows north toward the confluence with the North Fork of the Gunnison River and then flows west toward Delta, Colo., and the Uncompahgre River flows from its headwaters in the San Juan Mountains north toward Delta, Colo. The confluence of the Gunnison and Uncompahgre Rivers is near Delta, Colo., and from there, the Gunnison River flows north toward the Colorado River.

The climate of the study area is arid to semiarid and is characterized by cool to occasionally cold winters and hot summers. Temperatures at the Montrose Regional Airport ranged from a minimum of -13 degrees Fahrenheit $\left({ }^{\circ} \mathrm{F}\right)$ in December 1998 to a maximum of $101^{\circ} \mathrm{F}$ in July 2002 (Western Regional Climate Center, 2017). Mean annual precipitation and snowfall in Delta, Colo., ranged from 7.92 inches (in.) (1900) to 14.9 in. (1999) (Western Regional Climate Center, 2017). Mean annual precipitation and snowfall in Montrose, Colo., ranged from 9.60 in. (1895) to 27.2 in. (2016) (Western Regional Climate Center, 2017). Annual evaporation was 58 in. at Montrose, Colo. (Farnsworth and Thompson, 1982).

Surficial deposits in the study area are of Quaternary age and consist of alluvial deposits, mudflow-dominated alluvial and alluvial-fan deposits, mixed debris-flow and 
alluvial-gravel deposits, eolian deposits, and mass-wasting deposits (Noe and others, 2007). The alluvial channel deposits of the Gunnison and Uncompahgre Rivers generally consist of moderately sorted, well-rounded cobbles and pebbles in a coarse sand matrix. Finer grained alluvial, mudflow, and alluvial-fan deposits form complex systems along tributary streams and in broad basins (Noe and others, 2007). Remnants of older abandoned river deposits and glacial outwash are preserved as mesa-capping gravel bodies composed of poorly sorted rock flour, sand, gravel, cobbles, and boulders (Noe and others, 2007; Butler and others, 1996).

Bedrock units are sedimentary rocks of the lower Cretaceous Dakota Sandstone and Burro Canyon Formation and the upper Cretaceous Mancos Shale (fig. 2). The Mancos Shale is the primary geologic material outcropping in the study area (Tweto, 1979). It is marine in origin and has a maximum thickness of about 4,500 ft in the lower Gunnison River Basin (Brooks and Ackerman, 1985). The unit consists of several stratigraphic members that include clayey to sandy to calcareous shale with minor limestone, sandstone, and bentonite beds and that are differentiated by lithofacies associations, diagnostic fossils, and biostratigraphy (Noe and others, 2007). In the Montrose East 7.5-minute quadrangle, which represents the area south of Montrose and east of the Uncompahgre River, there are 10 stratigraphic members identified in the Mancos Shale (fig. 2). The Mancos Shale is the lateral equivalent of the Niobrara Formation, Cody Shale, and Pierre Shale in Colorado, Montana, Nebraska, South Dakota, and Wyoming (Butler and others, 1996). The Mancos Shale and its lateral equivalents represent the accumulation of sediments deposited in the deeper parts of the Late Cretaceous epicontinental sea that covered much of the Western Interior region of North America (Izett and others, 1971; McGookey and others, 1972).

The Mancos Shale has weathered in stages through geologic time. Incipient weathering began approximately 20,000 years ago, when water levels lowered and resulted in the oxidation of pyrite and organic matter (Tuttle and others, 2014). Gypsum and soluble organic matter were formed during this process and are currently present in unsaturated weathered Mancos Shale (Tuttle and others, 2014). During the second (current) stage of weathering, the shale disaggregates to soil (Tuttle and others, 2014), weathering to a clayey, silty residuum containing abundant secondary gypsum in shale partings and fractures, pyrite concretions altered to limonite, and ash layers weathered to kaolinite and quartz that contains some jarosite (Butler and others, 1996). Soils derived from the Mancos Shale commonly are associated with elevated concentrations of salt and selenium (Butler and others, 1996; Morrison and others, 2012; Mast and others, 2014).

Land use in the study area is primarily agriculture (fig. 3). Irrigation water is supplied by Reclamation's Uncompahgre Project (Reclamation, 2011), which delivers irrigation water to 76,000 acres of agricultural land, including the east side of the Uncompahgre River. The project includes the Taylor Park Dam and Reservoir, the Gunnison Tunnel, 7 diversion

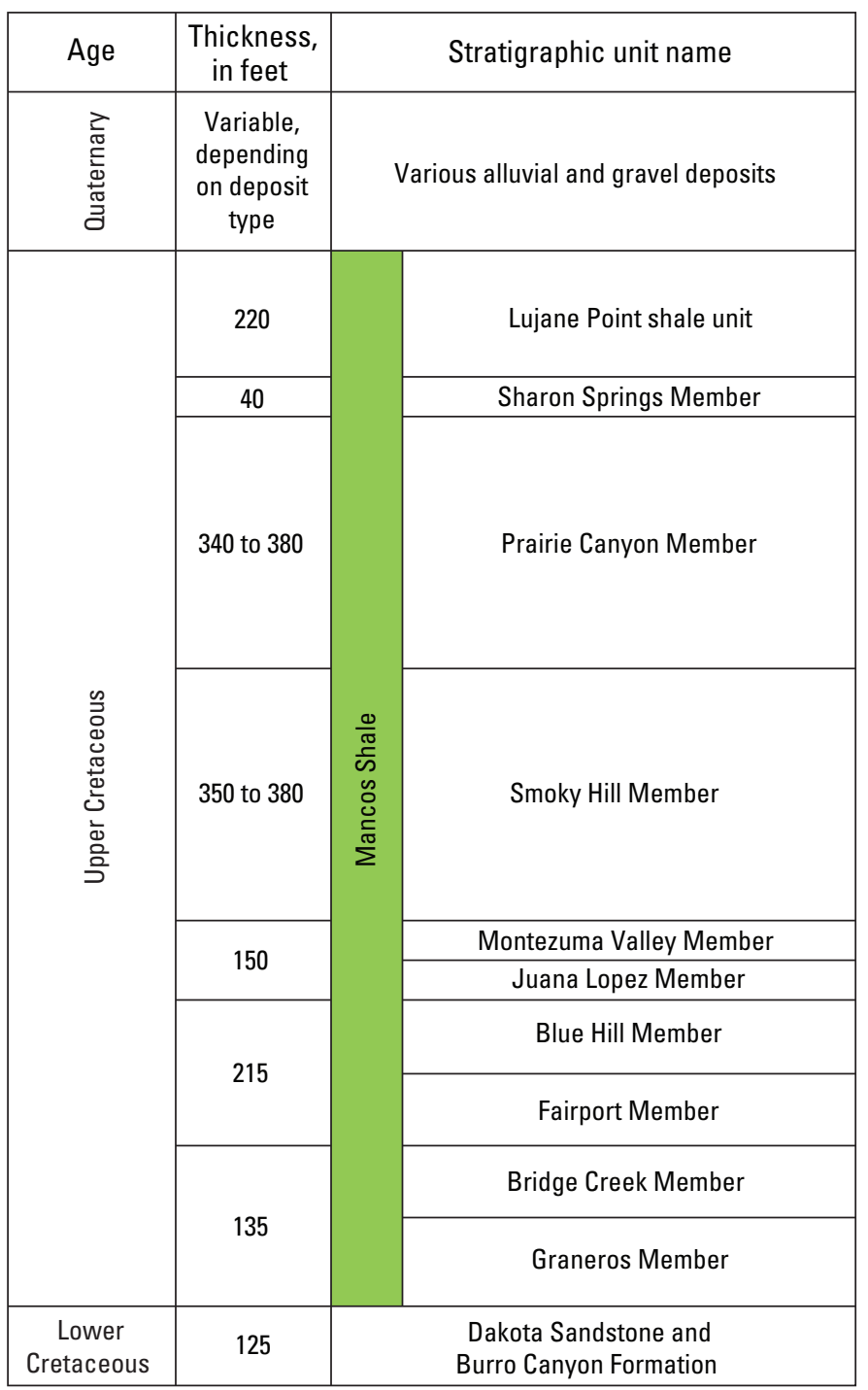

Figure 2. Generalized stratigraphic column for the lower Gunnison River Basin, Colorado. Modified from Noe and others (2007).

dams, 128 miles (mi) of main canals, $438 \mathrm{mi}$ of laterals, and $216 \mathrm{mi}$ of drains (Reclamation, 2017). The primary population centers in the study area are Delta, Olathe, and Montrose. Increasingly, agricultural land is being converted to residential and other urban land uses in the study area (Gunnison Basin Selenium Task Force, 2017).

\section{Methods}

This section provides details on the development of the groundwater-monitoring network, methods of sample collection and laboratory analysis, data analysis methods, quality assurance and quality control, hydrogeologic mapping, water-level measurements, single-hole aquifer tests and analysis, the watertable fluctuation method, groundwater-age calculations, and loading calculations that are presented in this report. 


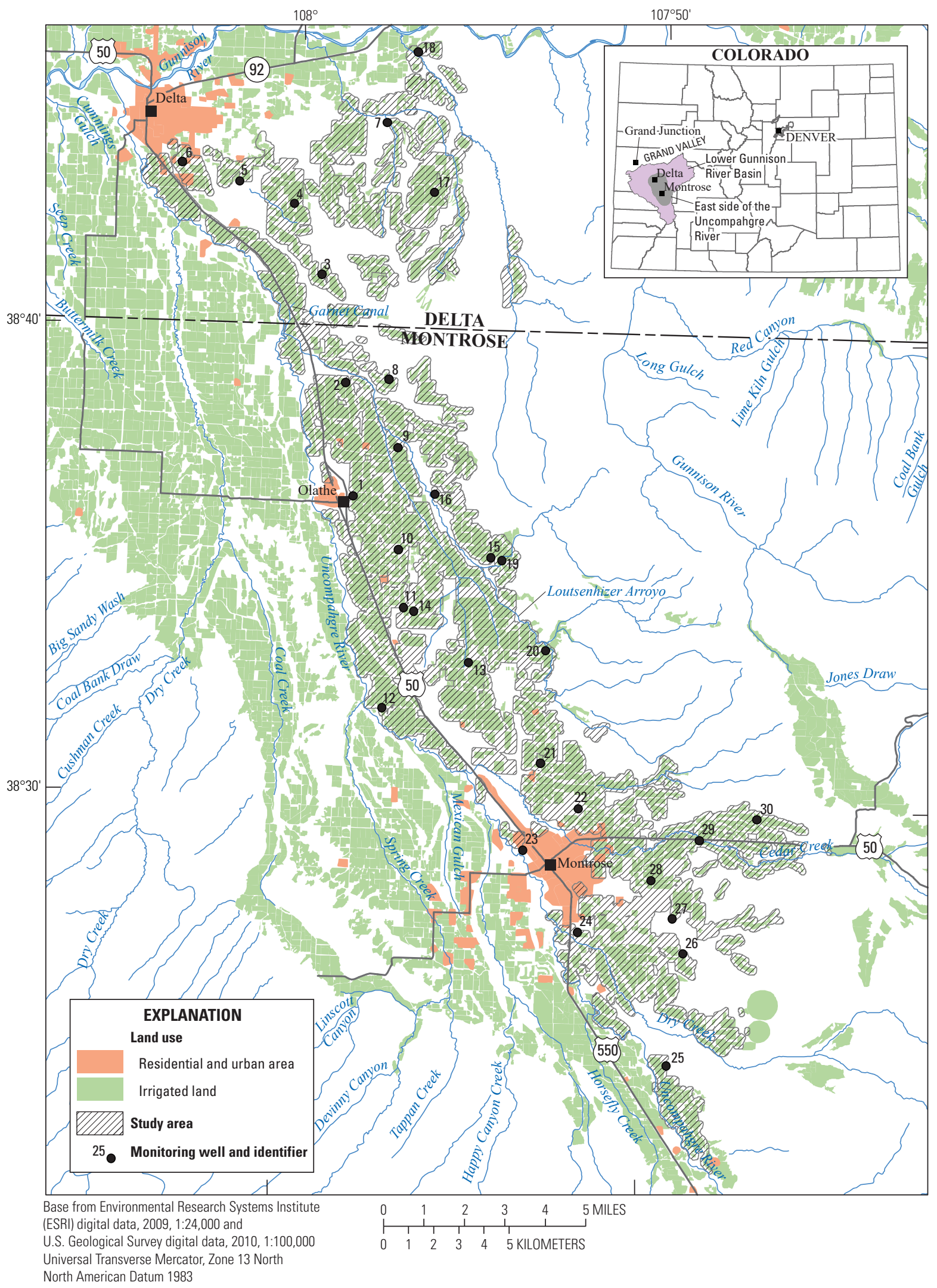

Figure 3. Land use of study area and location of monitoring wells, east side of the Uncompahgre River, lower Gunnison River Basin, Colorado. Land use data from Colorado Decision Support System (2013). 


\section{Groundwater Monitoring Network}

A 30-well groundwater-monitoring network on the east side of the Uncompahgre River Basin was designed to provide a statistically robust network of monitoring wells to characterize the shallow groundwater system (Thomas and Arnold, 2015) (fig. 3). The network design was created by using a computerized technique (Scott, 1990) that generates a random distribution of potential groundwater-sampling sites. The random site-selection process divided the study area into 30 equal-area polygons and then generated three potential groundwater-monitoring sites in each polygon: a primary, secondary, and tertiary site. When establishing sites, the primary site was considered first, followed by the secondary and tertiary sites (Thomas and Arnold, 2015). Final well locations were established based on landowner permission and site accessibility. Monitoring wells were drilled and installed during 2012 and 2014 (table 1); the process is described in Thomas and Arnold (2015) and Thomas (2015). The boreholes were drilled with a truckmounted drilling rig and advanced with 4.25-in. inside-diameter hollow-stem augers. Wells were constructed with 2-in.-diameter schedule- 40 polyvinyl-chloride casing and completed with a protective steel surface casing with a locking cap. Well screens were either 5 or $10 \mathrm{ft}$ long and were installed near the bottom of the saturated thickness. Split-spoon samples of geologic materials were collected to correctly identify formation intervals for well installation. Lithologic logs were developed based on visual inspection of split-spoon samples and auger cuttings (Thomas and Arnold, 2015; Thomas, 2015). Of the 12 holes drilled in October and November 2012, three were dry (well identifiers 8,11, and 13), indicating discontinuous saturated conditions in the study area (Thomas and Arnold, 2015). All 30 monitoring wells were developed to remove mud and any foreign material and to help improve the hydraulic connection of the well with the aquifer. Well development information and well construction diagrams are provided for each well in Thomas and Arnold (2015) and Thomas (2015).

\section{Water-Quality Sample Collection, Processing, and Analysis}

Monitoring wells were sampled on multiple occasions to assess groundwater quality, sources of recharge, and groundwater age. The first 10 wells that were installed were sampled during the irrigation season (August) of 2013, and the entire 30 -well network was sampled during the irrigation season (August) of 2014 and again during the nonirrigation season (March) of 2015 (table 2). Samples were analyzed to determine field properties and to determine the concentration of major ions, trace elements, selenium speciation (selenate and selenite), nutrients, dissolved organic carbon, stable hydrogen and oxygen isotopes in water (delta oxygen- $18\left[\delta^{2} \mathrm{H}-\mathrm{H}_{2} \mathrm{O}\right]$ and deuteriumprotium ratio $\left.\left[\delta^{18} \mathrm{O}-\mathrm{H}_{2} \mathrm{O}\right]\right)$ referred to as water isotopes, stable nitrogen and oxygen isotopes in nitrate (delta Nitrogen-15 in nitrate fraction $\left[\delta^{15} \mathrm{~N}-\mathrm{NO}_{3}\right]$ and delta Oxygen-18 in nitrate fraction $\left[\delta^{18} \mathrm{O}-\mathrm{NO}_{3}\right]$ ) referred to as nitrate isotopes, tritium, noble gases, sulfur hexafluoride ( $\mathrm{SF}_{6}$ ), and dissolved gases (table 3). Reporting limit based on a detection limit calculated by any of the detection limit procedures (Williams and Foreman, 2015). Water temperature, specific conductance, dissolved oxygen, and $\mathrm{pH}$ were measured by using a multiparameter field meter. Alkalinity was titrated in the field by incremental titration by using 1.6 normal sulfuric acid (USGS, variously dated). Turbidity was measured in the field by using a Hach 2100P portable turbidimeter. Data on water isotopes $\left(\delta^{2} \mathrm{H}-\mathrm{H}_{2} \mathrm{O}\right.$ and $\left.\delta^{18} \mathrm{O}-\mathrm{H}_{2} \mathrm{O}\right)$, tritium, noble gases, $\mathrm{SF}_{6}$, and dissolved gas were collected at selected wells based on the hydrogeologic unit in which the well was completed. All data collected are available through the USGS National Water Information System (NWIS) (USGS, 2017).

Groundwater-quality samples were collected according to standard USGS protocols for sampling monitoring wells as described in the "National Field Manual for the Collection of Water-Quality Data" (USGS, variously dated). Either a peristaltic pump or a submersible, positive-pressure pump was used to sample wells, depending on well conditions and constituent requirements. For tritium, $\mathrm{SF}_{6}$, and dissolved gases, it was necessary to use a positive-pressure pump to obtain a sample that has not been exposed to the atmosphere. Water samples were collected from monitoring wells after field properties had stabilized, as described in the "National Field Manual for the Collection of Water-Quality Data" (USGS, variously dated). Samples for the analysis of alkalinity, major ions, trace elements, nutrients, and dissolved organic carbon were filtered at the time of collection by using a disposable 0.45-micrometer capsule filter. Samples were collected in precleaned plastic bottles, with the exception of dissolved organic carbon, which was collected in a baked amber glass bottle. Samples collected for cation and trace-element analysis were acidified in the field to a $\mathrm{pH}$ less than 2 by using nitric acid. Samples collected for dissolved organic carbon analysis were acidified in the field by using sulfuric acid. Samples collected for nutrient and dissolved organic carbon analysis were kept chilled on ice until delivered to the laboratory.

Groundwater was also sampled for other constituents including water isotopes, nitrate isotopes, tritium, noble gases, $\mathrm{SF}_{6}$, and dissolved gases. Samples for analysis of water isotopes $\left(\delta^{2} \mathrm{H}-\mathrm{H}_{2} \mathrm{O}\right.$ and $\left.\delta^{18} \mathrm{O}-\mathrm{H}_{2} \mathrm{O}\right)$ (table 3 ) were unfiltered and collected in glass bottles with polyseal caps that were secured with electrical tape (USGS, 2015a). In addition to groundwater samples, four surface-water sites were sampled for water isotopes in which samples were collected as a single vertical at the centroid of flow with a 1-liter sample bottle. Samples for analysis of nitrate isotopes $\left(\delta^{15} \mathrm{~N}-\mathrm{NO}_{3}\right.$ and $\left.\delta^{18} \mathrm{O}-\mathrm{NO}_{3}\right)$ were first passed through a 0.45 -micrometer filter into a 60 -milliliter syringe with a 0.2-micrometer filter (USGS, 2015a). Filtrate was then collected into a 4-ounce amber polyethylene bottle and preserved with potassium hydroxide pellets (typically, 3 pellets were used); the cap was secured with electrical tape, and the bottle was chilled on ice until delivered to the laboratory. Samples for tritium analysis were unfiltered and collected in plastic bottles with polyseal caps and then secured with electrical tape (Hunt, 2015). Samples for noble-gas analysis (table 3) were unfiltered and collected in copper tubes that were sealed on both ends with pinch clamps (Hunt, 2015). The copper tubes were connected in line with the pump discharge to prevent atmospheric 
Table 1. Location, construction, and geologic information for monitoring wells, east side of the Uncompahgre River, lower Gunnison River Basin, Colorado.

[USGS, U.S. Geological Survey; DDMMSS, degrees, minutes, seconds. Coordinate datum North American Datum of 1983. Altitude datum North American Vertical Datum 1988. Gray shading indicates sites where pressure transducers were installed to measure depth to water in 15-minute intervals (continuous data). Measuring point height given in feet above land surface. All depths given in feet below land surface. Negative depth values indicate feet above land surface]

\begin{tabular}{|c|c|c|c|c|c|c|c|c|c|c|c|c|c|c|c|c|c|}
\hline $\begin{array}{c}\text { Well } \\
\text { identifier }\end{array}$ & $\begin{array}{l}\text { USGS site } \\
\text { identification } \\
\text { number }\end{array}$ & $\begin{array}{l}\text { Latitude } \\
\text { (DDMMSS) }\end{array}$ & $\begin{array}{l}\text { Longitude } \\
\text { (DDDMMSS) }\end{array}$ & $\begin{array}{c}\text { Date } \\
\text { completed }\end{array}$ & $\begin{array}{l}\text { Land- } \\
\text { surface } \\
\text { altitude } \\
\text { (feet) }\end{array}$ & $\begin{array}{c}\text { Depth to } \\
\text { water at } \\
\text { time of } \\
\text { drilling }\end{array}$ & $\begin{array}{l}\text { Measuring } \\
\text { point height }\end{array}$ & $\begin{array}{l}\text { Depth to } \\
\text { top of } \\
\text { screen }\end{array}$ & $\begin{array}{c}\text { Depth to } \\
\text { bottom of } \\
\text { screen }\end{array}$ & $\begin{array}{c}\text { Depth to } \\
\text { top of } \\
\text { annular } \\
\text { seal }\end{array}$ & $\begin{array}{c}\text { Depth to } \\
\text { top of } \\
\text { sand } \\
\text { pack }\end{array}$ & $\begin{array}{c}\text { Depth to } \\
\text { bottom } \\
\text { of sand } \\
\text { pack }\end{array}$ & $\begin{array}{l}\text { Total } \\
\text { well } \\
\text { depth }\end{array}$ & $\begin{array}{l}\text { Geologic interval well } \\
\text { screened in }\end{array}$ & $\begin{array}{c}\text { Depth to } \\
\text { top of } \\
\text { water } \\
\text { bearing } \\
\text { zone' }\end{array}$ & $\begin{array}{c}\text { Depth to } \\
\text { bottom } \\
\text { of water } \\
\text { bearing } \\
\text { zone' }\end{array}$ & $\begin{array}{c}\text { Thickness } \\
\text { of water } \\
\text { bearing } \\
\text { zone, feet }\end{array}$ \\
\hline 1 & 383626107581501 & 383626 & 1075815 & $4 / 26 / 2014$ & 5,416 & 8.5 & 2.4 & 15.7 & 20.7 & 2.0 & 14.0 & 21.0 & 21.0 & weathered Mancos Shale & 18.0 & 20.0 & 2.0 \\
\hline 2 & 383852107583301 & 383852 & 1075833 & $4 / 14 / 2014$ & 5,286 & 7.7 & 3.1 & 6.5 & 11.5 & 2.0 & 5.0 & 13.5 & 13.5 & surficial deposits & 8.5 & 13.5 & 5.0 \\
\hline 3 & 384110107591801 & 384110 & 1075918 & $4 / 11 / 2014$ & 5,245 & 7.3 & 2.6 & 7.2 & 12.2 & 2.0 & 6.0 & 14.7 & 14.7 & surficial deposits & 0.0 & 14.7 & 14.7 \\
\hline 4 & 384240108000701 & 384240 & 1080007 & $4 / 24 / 2014$ & 5,190 & 11.1 & 2.2 & 17.4 & 22.4 & 2.0 & 15.2 & 22.7 & 22.7 & weathered Mancos Shale & 17.7 & 21.3 & 3.6 \\
\hline 5 & 384306108013801 & 384306 & 1080138 & $4 / 24 / 2014$ & 5,164 & 20.4 & 2.9 & 21.7 & 26.7 & 2.0 & 19.0 & 27.0 & 27.0 & weathered Mancos Shale & 21.0 & 25.0 & 4.0 \\
\hline 6 & 384329108031301 & 384329 & 1080313 & 6/9/2014 & 5,094 & 29.5 & 3.0 & 34.5 & 44.5 & 2.0 & 30.0 & 46.5 & 46.5 & surficial deposits & 28.4 & 36.5 & 8.1 \\
\hline 7 & 384428107573901 & 384428 & 1075739 & $4 / 23 / 2014$ & 5,087 & 19.8 & 2.5 & 22.2 & 27.2 & 2.0 & 20.5 & 28.2 & 28.2 & surficial deposits & 26.7 & 28.2 & 1.5 \\
\hline 8 & 383844107572801 & 383844 & 1075728 & $11 / 11 / 2012$ & 5,302 & 11.8 & 2.8 & 15.1 & 20.0 & 1.4 & 14.1 & 21.6 & 24.0 & surficial deposits & 11.1 & 24.0 & 12.9 \\
\hline 9 & 383730107570501 & 383730 & 1075705 & $4 / 25 / 2014$ & 5,364 & 9.5 & 2.5 & 26.0 & 31.0 & 2.0 & 24.5 & 31.3 & 31.3 & weathered Mancos Shale & 28.3 & 31.2 & 2.9 \\
\hline 10 & 383520107565901 & 383520 & 1075659 & 4/9/2014 & 5,483 & 14.3 & 3.0 & 16.0 & 21.0 & 2.0 & 14.5 & 23.5 & 23.5 & weathered Mancos Shale & 18.5 & 19.3 & 0.8 \\
\hline 11 & 383405107564701 & 383405 & 1075647 & $11 / 10 / 2012$ & 5,559 & 6.0 & 2.3 & 7.2 & 16.9 & 1.8 & 6.0 & 19.0 & 19.0 & surficial deposits & 6.0 & 19.0 & 13.0 \\
\hline 12 & 383156107571701 & 383156 & 1075717 & $11 / 11 / 2012$ & 5,544 & 9.0 & 1.8 & 11.1 & 16.0 & 1.0 & 5.5 & 19.0 & 19.0 & surficial deposits & 11.1 & 19.0 & 7.9 \\
\hline 13 & 383257107545801 & 383257 & 1075458 & $11 / 10 / 2012$ & 5,604 & 8.2 & 2.6 & 10.1 & 15.0 & 0.5 & 8.5 & 18.0 & 19.0 & surficial deposits & 7.5 & 15.0 & 7.5 \\
\hline 14 & 383401107563001 & 383401 & 1075630 & $4 / 27 / 2014$ & 5,576 & 22.2 & 2.9 & 26.7 & 36.7 & 2.0 & 25.0 & 42.0 & 42.0 & weathered Mancos Shale & 33.0 & 42.0 & 9.0 \\
\hline 15 & 383513107542601 & 383513 & 1075426 & $10 / 27 / 2012$ & 5,506 & 9.7 & 2.6 & 17.9 & 27.6 & 0.8 & 15.7 & 26.6 & 31.7 & surficial deposits & 18.0 & 31.7 & 13.7 \\
\hline 16 & 383632107560201 & 383632 & 1075602 & $10 / 28 / 2012$ & 5,409 & 21.1 & 2.8 & 24.3 & 34.0 & 1.0 & 21.6 & 36.3 & 39.3 & surficial deposits & 23.3 & 39.3 & 16.0 \\
\hline 17 & 384300107561801 & 384300 & 1075618 & $4 / 11 / 2014$ & 5,227 & 9.2 & 3.0 & 16.3 & 21.3 & 2.0 & 13.8 & 23.3 & 23.3 & weathered Mancos Shale & 18.3 & 21.8 & 3.5 \\
\hline 18 & 384559107565201 & 384559 & 1075652 & $4 / 23 / 2014$ & 5,060 & 14.8 & 2.9 & 12.5 & 17.5 & 2.0 & 10.5 & 18.5 & 18.5 & surficial deposits & 11.5 & 18.5 & 7.0 \\
\hline 19 & 383510107540801 & 383510 & 1075408 & $4 / 26 / 2014$ & 5,531 & 7.5 & 2.8 & 7.5 & 17.5 & 2.0 & 6.0 & 18.5 & 18.5 & surficial deposits & 8.1 & 18.5 & 10.4 \\
\hline 20 & 383315107525201 & 383315 & 1075252 & $10 / 26 / 2012$ & 5,657 & 14.0 & 2.9 & 19.2 & 28.9 & 1.6 & 17.0 & 31.0 & 34.2 & surficial deposits & 19.2 & 28.9 & 9.7 \\
\hline 21 & 383051107525501 & 383051 & 1075255 & $4 / 28 / 2014$ & 5,743 & 8.5 & 2.5 & 12.1 & 17.1 & 2.0 & 10.0 & 18.1 & 18.1 & weathered Mancos Shale & 14.0 & 16.5 & 2.5 \\
\hline 22 & 382954107515101 & 382954 & 1075151 & $4 / 14 / 2014$ & 5,814 & 21.3 & -0.2 & 22.0 & 33.0 & 2.0 & 20.0 & 33.8 & 33.8 & surficial deposits & 0.0 & 29.8 & 29.8 \\
\hline 23 & 382859107531901 & 382859 & 1075319 & 6/9/2014 & 5,762 & 8.1 & -0.3 & 14.5 & 19.5 & 2.0 & 6.0 & 20.9 & 21.1 & surficial deposits & 12.0 & 21.1 & 9.1 \\
\hline 24 & 382715107514501 & 382715 & 1075145 & $6 / 13 / 2013$ & 5,876 & 14.8 & 3.1 & 17.0 & 21.9 & 0.5 & 15.3 & 24.0 & 27.1 & surficial deposits & 16.8 & 27.1 & 10.3 \\
\hline 25 & 382427107491401 & 382427 & 1074914 & $4 / 28 / 2014$ & 6,121 & 9.8 & 2.0 & 8.2 & 13.2 & 2.0 & 6.0 & 16.2 & 16.2 & weathered Mancos Shale & 8.0 & 16.2 & 8.2 \\
\hline 26 & 382656107500701 & 382656 & 1075007 & $10 / 25 / 2012$ & 5,954 & 10.4 & 2.5 & 15.0 & 19.9 & 1.0 & 13.1 & 22.0 & 24.4 & surficial deposits & 14.0 & 20.6 & 6.6 \\
\hline 27 & 382736107491201 & 382736 & 1074912 & $4 / 10 / 2014$ & 6,021 & 2.1 & 3.0 & 21.3 & 26.3 & 2.0 & 19.6 & 28.3 & 28.3 & weathered Mancos Shale & 18.6 & 19.0 & 0.4 \\
\hline 28 & 382824107494801 & 382824 & 1074948 & $10 / 25 / 2012$ & 5,995 & 9.9 & 2.7 & 15.7 & 20.6 & 1.0 & 13.8 & 20.8 & 20.8 & weathered Mancos Shale & 19.0 & 20.8 & 1.8 \\
\hline 29 & 382917107483101 & 382917 & 1074831 & $4 / 12 / 2014$ & 6,106 & 7.0 & 2.0 & 7.5 & 12.5 & 2.0 & 5.5 & 15.0 & 15.0 & surficial deposits & 0.0 & 18.0 & 18.0 \\
\hline 30 & 382947107465801 & 382947 & 1074658 & $4 / 15 / 2014$ & 6,247 & 5.1 & 2.7 & 14.1 & 19.1 & 2.0 & 12.2 & 19.5 & 19.5 & weathered Mancos Shale & 18.2 & 21.2 & 3.0 \\
\hline
\end{tabular}

${ }^{1}$ From lithologic logs in Thomas and Arnold (2015) and Thomas (2015). 
Table 2. Summary of sampling events and analysis done at monitoring wells, east side of the Uncompahgre River, lower Gunnison River Basin, Colorado, 2013-16.

[A, August 2013; B, August 2014; C, March 2015; --, not collected]

\begin{tabular}{|c|c|c|c|c|c|c|c|c|c|c|c|c|}
\hline \multirow{2}{*}{$\begin{array}{c}\text { Well } \\
\text { identifier }\end{array}$} & \multirow{2}{*}{$\begin{array}{c}\text { Field } \\
\text { properties }\end{array}$} & \multirow{2}{*}{$\begin{array}{c}\text { Major } \\
\text { ions }\end{array}$} & \multicolumn{2}{|c|}{ Trace elements } & \multicolumn{2}{|c|}{$\begin{array}{l}\text { Nutrients and } \\
\text { dissolved } \\
\text { organic carbon }\end{array}$} & \multirow{2}{*}{$\begin{array}{c}\text { Water } \\
\text { isotopes }\end{array}$} & \multirow{2}{*}{$\begin{array}{c}\text { Nitrate } \\
\text { isotopes }\end{array}$} & \multirow{2}{*}{$\begin{array}{c}\text { Noble } \\
\text { gas }\end{array}$} & \multirow{2}{*}{$\begin{array}{l}\text { Sulfur } \\
\text { Hexa- } \\
\text { fluoride }\end{array}$} & \multirow{2}{*}{ Tritium } & \multirow{2}{*}{$\begin{array}{c}\text { Dissolved } \\
\text { gas }\end{array}$} \\
\hline & & & $\begin{array}{c}\text { Trace } \\
\text { elements }\end{array}$ & $\begin{array}{c}\text { Selenate } \\
\text { and } \\
\text { selenite }\end{array}$ & Nutrients & $\begin{array}{l}\text { Dissolved } \\
\text { organic } \\
\text { carbon }\end{array}$ & & & & & & \\
\hline 1 & $\mathrm{~B}, \mathrm{C}$ & $\mathrm{B}, \mathrm{C}$ & $\mathrm{B}, \mathrm{C}$ & $\mathrm{B}, \mathrm{C}$ & $\mathrm{B}, \mathrm{C}$ & $\mathrm{B}, \mathrm{C}$ & $\mathrm{B}$ & $\mathrm{B}$ & $\mathrm{B}^{1}$ & $\mathrm{~B}$ & -- & $\mathrm{B}^{1}$ \\
\hline 2 & $\mathrm{~B}, \mathrm{C}$ & $\mathrm{B}, \mathrm{C}$ & $\mathrm{B}, \mathrm{C}$ & $\mathrm{B}, \mathrm{C}$ & $\mathrm{B}, \mathrm{C}$ & $\mathrm{B}, \mathrm{C}$ & $\mathrm{B}, \mathrm{C}$ & $\mathrm{B}, \mathrm{C}$ & $\mathrm{C}$ & $\mathrm{C}$ & $\mathrm{C}$ & $\mathrm{C}$ \\
\hline 3 & $\mathrm{~B}, \mathrm{C}$ & $\mathrm{B}, \mathrm{C}$ & $\mathrm{B}, \mathrm{C}$ & $\mathrm{B}, \mathrm{C}$ & $\mathrm{B}, \mathrm{C}$ & $\mathrm{B}, \mathrm{C}$ & $\mathrm{B}, \mathrm{C}$ & -- & -- & $\mathrm{C}$ & $\mathrm{C}$ & $\mathrm{C}$ \\
\hline 4 & $\mathrm{~B}, \mathrm{C}$ & $\mathrm{B}, \mathrm{C}$ & $\mathrm{B}, \mathrm{C}$ & $\mathrm{B}, \mathrm{C}$ & $\mathrm{B}, \mathrm{C}$ & $\mathrm{B}, \mathrm{C}$ & B & -- & -- & -- & -- & -- \\
\hline 5 & $\mathrm{~B}, \mathrm{C}$ & $\mathrm{B}, \mathrm{C}$ & $\mathrm{B}, \mathrm{C}$ & $\mathrm{B}, \mathrm{C}$ & $\mathrm{B}, \mathrm{C}$ & $\mathrm{B}, \mathrm{C}$ & B & B & B & $\mathrm{B}$ & -- & B \\
\hline 6 & $\mathrm{~B}, \mathrm{C}$ & $\mathrm{B}, \mathrm{C}$ & $\mathrm{B}, \mathrm{C}$ & $\mathrm{B}, \mathrm{C}$ & $\mathrm{B}, \mathrm{C}$ & $\mathrm{B}, \mathrm{C}$ & $\mathrm{B}, \mathrm{C}$ & $\mathrm{B}, \mathrm{C}$ & -- & $\mathrm{C}$ & $\mathrm{C}$ & $\mathrm{B}, \mathrm{C}$ \\
\hline 7 & $\mathrm{~B}, \mathrm{C}$ & $\mathrm{B}, \mathrm{C}$ & $\mathrm{B}, \mathrm{C}$ & $\mathrm{B}, \mathrm{C}$ & $\mathrm{B}, \mathrm{C}$ & $\mathrm{B}, \mathrm{C}$ & $\mathrm{B}, \mathrm{C}$ & B & $\mathrm{B}^{1}$ & $\mathrm{~B}$ & -- & $\mathrm{B}^{1}$ \\
\hline 8 & $\mathrm{~A}, \mathrm{~B}, \mathrm{C}$ & $\mathrm{A}, \mathrm{B}, \mathrm{C}$ & $\mathrm{A}, \mathrm{B}, \mathrm{C}$ & $\mathrm{A}, \mathrm{B}, \mathrm{C}$ & $\mathrm{A}, \mathrm{B}, \mathrm{C}$ & $\mathrm{B}, \mathrm{C}$ & B & $\mathrm{B}, \mathrm{C}$ & $\mathrm{C}$ & $\mathrm{C}$ & $\mathrm{C}$ & $\mathrm{B}, \mathrm{C}$ \\
\hline 9 & B, C & $\mathrm{B}, \mathrm{C}$ & $\mathrm{B}, \mathrm{C}$ & $\mathrm{B}, \mathrm{C}$ & B, C & $\mathrm{B}, \mathrm{C}$ & $\mathrm{B}, \mathrm{C}$ & $\mathrm{B}, \mathrm{C}$ & -- & $\mathrm{C}$ & $\mathrm{C}$ & $\mathrm{C}$ \\
\hline 10 & $\mathrm{~B}, \mathrm{C}$ & $\mathrm{B}, \mathrm{C}$ & $\mathrm{B}, \mathrm{C}$ & $\mathrm{B}, \mathrm{C}$ & $\mathrm{B}, \mathrm{C}$ & $\mathrm{B}, \mathrm{C}$ & $\mathrm{B}, \mathrm{C}$ & $\mathrm{C}$ & -- & $\mathrm{C}$ & $\mathrm{C}$ & $\mathrm{B}, \mathrm{C}$ \\
\hline 11 & $\mathrm{~A}, \mathrm{~B}, \mathrm{C}$ & $\mathrm{A}, \mathrm{B}, \mathrm{C}$ & $\mathrm{A}, \mathrm{B}, \mathrm{C}$ & $\mathrm{A}, \mathrm{B}, \mathrm{C}$ & $\mathrm{A}, \mathrm{B}, \mathrm{C}$ & $\mathrm{B}, \mathrm{C}$ & B & B & -- & -- & -- & B \\
\hline 12 & $\mathrm{~A}, \mathrm{~B}, \mathrm{C}$ & $\mathrm{A}, \mathrm{B}, \mathrm{C}$ & $\mathrm{A}, \mathrm{B}, \mathrm{C}$ & $\mathrm{A}, \mathrm{B}, \mathrm{C}$ & $\mathrm{A}, \mathrm{B}, \mathrm{C}$ & $\mathrm{B}, \mathrm{C}$ & B & -- & -- & -- & -- & B \\
\hline 13 & $\mathrm{~A}, \mathrm{~B}, \mathrm{C}$ & $\mathrm{A}, \mathrm{B}, \mathrm{C}$ & $\mathrm{A}, \mathrm{B}, \mathrm{C}$ & $\mathrm{A}, \mathrm{B}, \mathrm{C}$ & $\mathrm{A}, \mathrm{B}, \mathrm{C}$ & $\mathrm{B}, \mathrm{C}$ & B & B & -- & -- & -- & B \\
\hline 14 & B, C & $\mathrm{B}, \mathrm{C}$ & $\mathrm{B}, \mathrm{C}$ & $\mathrm{B}, \mathrm{C}$ & B, C & $\mathrm{B}, \mathrm{C}$ & B & -- & -- & -- & -- & $\mathrm{B}^{1}$ \\
\hline 15 & $\mathrm{~A}, \mathrm{~B}, \mathrm{C}$ & $\mathrm{A}, \mathrm{B}, \mathrm{C}$ & $\mathrm{A}, \mathrm{B}, \mathrm{C}$ & $\mathrm{A}, \mathrm{B}, \mathrm{C}$ & $\mathrm{A}, \mathrm{B}, \mathrm{C}$ & $\mathrm{B}, \mathrm{C}$ & $\mathrm{B}, \mathrm{C}$ & $\mathrm{B}, \mathrm{C}$ & -- & $\mathrm{C}$ & $\mathrm{C}$ & $\mathrm{C}$ \\
\hline 16 & $\mathrm{~A}, \mathrm{~B}, \mathrm{C}$ & $\mathrm{A}, \mathrm{B}, \mathrm{C}$ & $\mathrm{A}, \mathrm{B}, \mathrm{C}$ & $\mathrm{A}, \mathrm{B}, \mathrm{C}$ & $\mathrm{A}, \mathrm{B}, \mathrm{C}$ & $\mathrm{B}, \mathrm{C}$ & B & -- & -- & -- & -- & -- \\
\hline 17 & $\mathrm{~B}, \mathrm{C}$ & $\mathrm{B}, \mathrm{C}$ & $\mathrm{B}, \mathrm{C}$ & $\mathrm{B}, \mathrm{C}$ & B, C & $\mathrm{B}, \mathrm{C}$ & $\mathrm{B}, \mathrm{C}$ & $\mathrm{B}, \mathrm{C}$ & $\mathrm{C}$ & $\mathrm{C}$ & $\mathrm{C}$ & $\mathrm{C}$ \\
\hline 18 & $\mathrm{~B}, \mathrm{C}$ & $\mathrm{B}, \mathrm{C}$ & $\mathrm{B}, \mathrm{C}$ & $\mathrm{B}, \mathrm{C}$ & $\mathrm{B}, \mathrm{C}$ & $\mathrm{B}, \mathrm{C}$ & B & -- & -- & $\mathrm{C}$ & -- & $\mathrm{C}$ \\
\hline 19 & B, C & $\mathrm{B}, \mathrm{C}$ & $\mathrm{B}, \mathrm{C}$ & B, C & B, C & $\mathrm{B}, \mathrm{C}$ & B & -- & -- & -- & -- & -- \\
\hline 20 & $\mathrm{~A}, \mathrm{~B}, \mathrm{C}$ & $\mathrm{A}, \mathrm{B}, \mathrm{C}$ & $\mathrm{A}, \mathrm{B}, \mathrm{C}$ & $\mathrm{A}, \mathrm{B}, \mathrm{C}$ & $\mathrm{A}, \mathrm{B}, \mathrm{C}$ & $\mathrm{B}, \mathrm{C}$ & B & B & -- & -- & -- & -- \\
\hline 21 & $\mathrm{~B}, \mathrm{C}$ & $\mathrm{B}, \mathrm{C}$ & $\mathrm{B}, \mathrm{C}$ & $\mathrm{B}, \mathrm{C}$ & $\mathrm{B}, \mathrm{C}$ & $\mathrm{B}, \mathrm{C}$ & B & -- & -- & -- & -- & -- \\
\hline 22 & $\mathrm{~B}, \mathrm{C}$ & $\mathrm{B}, \mathrm{C}$ & $\mathrm{B}, \mathrm{C}$ & $\mathrm{B}, \mathrm{C}$ & $\mathrm{B}, \mathrm{C}$ & $\mathrm{B}, \mathrm{C}$ & $\mathrm{B}, \mathrm{C}$ & $\mathrm{B}, \mathrm{C}$ & -- & $\mathrm{C}$ & $\mathrm{C}$ & $\mathrm{B}, \mathrm{C}$ \\
\hline 23 & B, C & $\mathrm{B}, \mathrm{C}$ & $\mathrm{B}, \mathrm{C}$ & $\mathrm{B}, \mathrm{C}$ & $\mathrm{B}, \mathrm{C}$ & $\mathrm{B}, \mathrm{C}$ & B & -- & -- & -- & -- & -- \\
\hline 24 & $\mathrm{~A}, \mathrm{~B}, \mathrm{C}$ & $\mathrm{A}, \mathrm{B}, \mathrm{C}$ & $\mathrm{A}, \mathrm{B}, \mathrm{C}$ & $\mathrm{A}, \mathrm{B}, \mathrm{C}$ & $\mathrm{A}, \mathrm{B}, \mathrm{C}$ & $\mathrm{B}, \mathrm{C}$ & B & -- & B & $\mathrm{B}$ & -- & B \\
\hline 25 & B, C & $\mathrm{B}, \mathrm{C}$ & $\mathrm{B}, \mathrm{C}$ & B, C & B, C & $\mathrm{B}, \mathrm{C}$ & $\mathrm{B}, \mathrm{C}$ & -- & $\mathrm{C}$ & $\mathrm{C}$ & $\mathrm{C}$ & $\mathrm{B}, \mathrm{C}$ \\
\hline 26 & $\mathrm{~A}, \mathrm{~B}, \mathrm{C}$ & $\mathrm{A}, \mathrm{B}, \mathrm{C}$ & $\mathrm{A}, \mathrm{B}, \mathrm{C}$ & $\mathrm{A}, \mathrm{B}, \mathrm{C}$ & $\mathrm{A}, \mathrm{B}, \mathrm{C}$ & $\mathrm{B}, \mathrm{C}$ & B & B & -- & -- & -- & -- \\
\hline 27 & B, C & $\mathrm{B}, \mathrm{C}$ & $\mathrm{B}, \mathrm{C}$ & $\mathrm{B}, \mathrm{C}$ & $\mathrm{B}, \mathrm{C}$ & $\mathrm{B}, \mathrm{C}$ & B & B & -- & -- & -- & B \\
\hline 28 & $\mathrm{~A}, \mathrm{~B}, \mathrm{C}$ & $\mathrm{A}, \mathrm{B}, \mathrm{C}$ & $\mathrm{A}, \mathrm{B}, \mathrm{C}$ & $\mathrm{A}, \mathrm{B}, \mathrm{C}$ & $\mathrm{A}, \mathrm{B}, \mathrm{C}$ & $\mathrm{B}, \mathrm{C}$ & B & B & -- & -- & -- & B \\
\hline 29 & $\mathrm{~B}, \mathrm{C}$ & $\mathrm{B}, \mathrm{C}$ & $\mathrm{B}, \mathrm{C}$ & $\mathrm{B}, \mathrm{C}$ & B, C & $\mathrm{B}, \mathrm{C}$ & B & -- & -- & -- & -- & -- \\
\hline 30 & $\mathrm{~B}, \mathrm{C}$ & $\mathrm{B}, \mathrm{C}$ & $\mathrm{B}, \mathrm{C}$ & $\mathrm{B}, \mathrm{C}$ & $\mathrm{B}, \mathrm{C}$ & $\mathrm{B}, \mathrm{C}$ & $\mathrm{B}$ & -- & -- & -- & -- & -- \\
\hline
\end{tabular}

${ }^{1}$ Samples were actively degassing because of high gas contents, resulting in uncertainty related to analytical results and use in interpretation of groundwater age. 
Table 3. Constituents, result units, reporting limit, and analyzing laboratories for water-quality samples.

[Reporting limit based on a detection limit calculated by any of the detection limit procedures. $\mathrm{mg} / \mathrm{L}$, milligram per liter; $\mu \mathrm{S} / \mathrm{cm}$, microseimens per centimeter; $\mu \mathrm{g} / \mathrm{L}$, microgram per liter; $\mathrm{CaCO}_{3}$; calcium carbonate; mrl, minimum reporting level; DLDQC, detection limit by DQCALC software: lowest concentration that with 90 percent confidence will be exceeded no more than 1 percent of the time when a blank sample is measured ( $\leq 1$ percent false positive risk). DLDQC equal to critical level "LC" by American Society for Testing and Materials (ASTM) D6091 approximately equals method detection limit (Williams and Foreman, 2015); NWQL, National Water Quality Laboratory; $\mathrm{NH}_{3}$, ammonia; $\mathrm{NO}_{2}$, nitrite; $\mathrm{NO}_{3}$, nitrate; lt-mdl, long-term method detection level; per mil, one part in one thousand parts; USGSCORL, U.S. Geological Survey Colorado Water Science Center Water-Quality Research Lab (Denver); --, not applicable; RSIL, Reston Stable Isotope Laboratory; NA, not applicable; $\mathrm{cm}^{3} \mathrm{STP} / \mathrm{g}\left(\mathrm{H}_{2} \mathrm{O}\right)$, cubic centimeter at standard temperature and pressure per gram of water; Hunt, U.S. Geological Survey Noble Gas laboratory; pptv, parts per trillion by volume]

\begin{tabular}{|c|c|c|c|c|c|}
\hline Constituent & $\begin{array}{l}\text { Parameter } \\
\text { code }\end{array}$ & Result units & $\begin{array}{c}\text { Report limit } \\
\text { type }\end{array}$ & $\begin{array}{l}\text { Reporting } \\
\text { limit }\end{array}$ & Laboratory \\
\hline \multicolumn{6}{|c|}{ Field properties } \\
\hline Specific conductance, water, unfiltered & 00095 & $\mu \mathrm{S} / \mathrm{cm}$ & $\mathrm{mrl}$ & 2.6 & Field \\
\hline Dissolved oxygen, water, unfiltered & 00300 & $\mathrm{mg} / \mathrm{L}$ & $\mathrm{mrl}$ & 0.1 & Field \\
\hline $\mathrm{pH}$, water, unfiltered & 00400 & standard unit & $\mathrm{mrl}$ & 0.1 & Field \\
\hline Turbidity, water, unfiltered & 63676 & $\begin{array}{c}\text { nephlometric } \\
\text { turbidity ratio-units } \\
\text { (NTRU) }\end{array}$ & $\mathrm{mrl}$ & 0.2 & Field \\
\hline \multicolumn{6}{|c|}{ Major ions } \\
\hline Bromide, water, filtered & 71870 & $\mathrm{mg} / \mathrm{L}$ & DLDQC & 0.01 & NWQL \\
\hline Magnesium, water, filtered & 00925 & $\mathrm{mg} / \mathrm{L}$ & DLDQC & 0.011 & NWQL \\
\hline Potassium, water, filtered & 00935 & $\mathrm{mg} / \mathrm{L}$ & DLDQC & 0.03 & NWQL \\
\hline Residue on evaporation (total dissolved solids) & 70300 & $\mathrm{mg} / \mathrm{L}$ & $\mathrm{mrl}$ & 20 & NWQL \\
\hline Silica, water, filtered & 00955 & $\mathrm{mg} / \mathrm{L}$ & DLDQC & 0.018 & NWQL \\
\hline Sodium, water, filtered & 00930 & $\mathrm{mg} / \mathrm{L}$ & DLDQC & 0.06 & NWQL \\
\hline Sulfate, water, filtered & 00945 & $\mathrm{mg} / \mathrm{L}$ & DLDQC & 0.02 & NWQL \\
\hline \multicolumn{6}{|c|}{ Trace elements } \\
\hline Aluminum, water, filtered & 01106 & $\mu \mathrm{g} / \mathrm{L}$ & DLDQC & 3 & NWQL \\
\hline Antimony, water, filtered & 01095 & $\mu \mathrm{g} / \mathrm{L}$ & DLDQC & 0.027 & NWQL \\
\hline Arsenic, water, filtered & 01000 & $\mu \mathrm{g} / \mathrm{L}$ & DLDQC & 0.1 & NWQL \\
\hline Copper, water, filtered & 01040 & $\mu \mathrm{g} / \mathrm{L}$ & DLDQC & 0.8 & NWQL \\
\hline Iron, water, filtered & 01046 & $\mu \mathrm{g} / \mathrm{L}$ & DLDQC & 4 & NWQL \\
\hline Lead, water, filtered & 01049 & $\mu \mathrm{g} / \mathrm{L}$ & DLDQC & 0.04 & NWQL \\
\hline Lithium, water, filtered & 01130 & $\mu \mathrm{g} / \mathrm{L}$ & DLDQC & 0.22 & NWQL \\
\hline Manganese, water, filtered & 01056 & $\mu \mathrm{g} / \mathrm{L}$ & DLDQC & 0.4 & NWQL \\
\hline Molybdenum, water, filtered & 01060 & $\mu \mathrm{g} / \mathrm{L}$ & DLDQC & 0.05 & NWQL \\
\hline Nickel, water, filtered & 01065 & $\mu \mathrm{g} / \mathrm{L}$ & DLDQC & 0.2 & NWQL \\
\hline Selenium, water, filtered & 01145 & $\mu \mathrm{g} / \mathrm{L}$ & DLDQC & 0.05 & NWQL \\
\hline Selenate, water, filtered ${ }^{1}$ & 67325 & $\mu \mathrm{g} / \mathrm{L}$ as selenium & - & 0.4 & USGSCORL \\
\hline Selenite, water, filtered & 67326 & $\mu \mathrm{g} / \mathrm{L}$ as selenium & - & 0.4 & USGSCORL \\
\hline Silver, water, filtered & 01075 & $\mu \mathrm{g} / \mathrm{L}$ & DLDQC & 0.02 & NWQL \\
\hline
\end{tabular}


Table 3. Constituents, result units, reporting limit, and analyzing laboratories for water-quality samples.-Continued

[Reporting limit based on a detection limit calculated by any of the detection limit procedures. $\mathrm{mg} / \mathrm{L}$, milligram per liter; $\mu \mathrm{S} / \mathrm{cm}$, microseimens per centimeter; $\mu \mathrm{g} / \mathrm{L}$, microgram per liter; $\mathrm{CaCO}_{3}$; calcium carbonate; mrl, minimum reporting level; DLDQC, detection limit by DQCALC software: lowest concentration that with 90 percent confidence will be exceeded no more than 1 percent of the time when a blank sample is measured ( $\leq 1$ percent false positive risk). DLDQC equal to critical level "LC" by American Society for Testing and Materials (ASTM) D6091 approximately equals method detection limit (Williams and Foreman, 2015); NWQL, National Water Quality Laboratory; $\mathrm{NH}_{3}$, ammonia; $\mathrm{NO}_{2}$, nitrite; $\mathrm{NO}_{3}$, nitrate; lt-mdl, long-term method detection level; per mil, one part in one thousand parts; USGSCORL, U.S. Geological Survey Colorado Water Science Center Water-Quality Research Lab (Denver); --, not applicable; RSIL, Reston Stable Isotope Laboratory; NA, not applicable; $\mathrm{cm}^{3} \mathrm{STP} / \mathrm{g}\left(\mathrm{H}_{2} \mathrm{O}\right)$, cubic centimeter at standard temperature and pressure per gram of water; Hunt, U.S. Geological Survey Noble Gas laboratory; pptv, parts per trillion by volume]

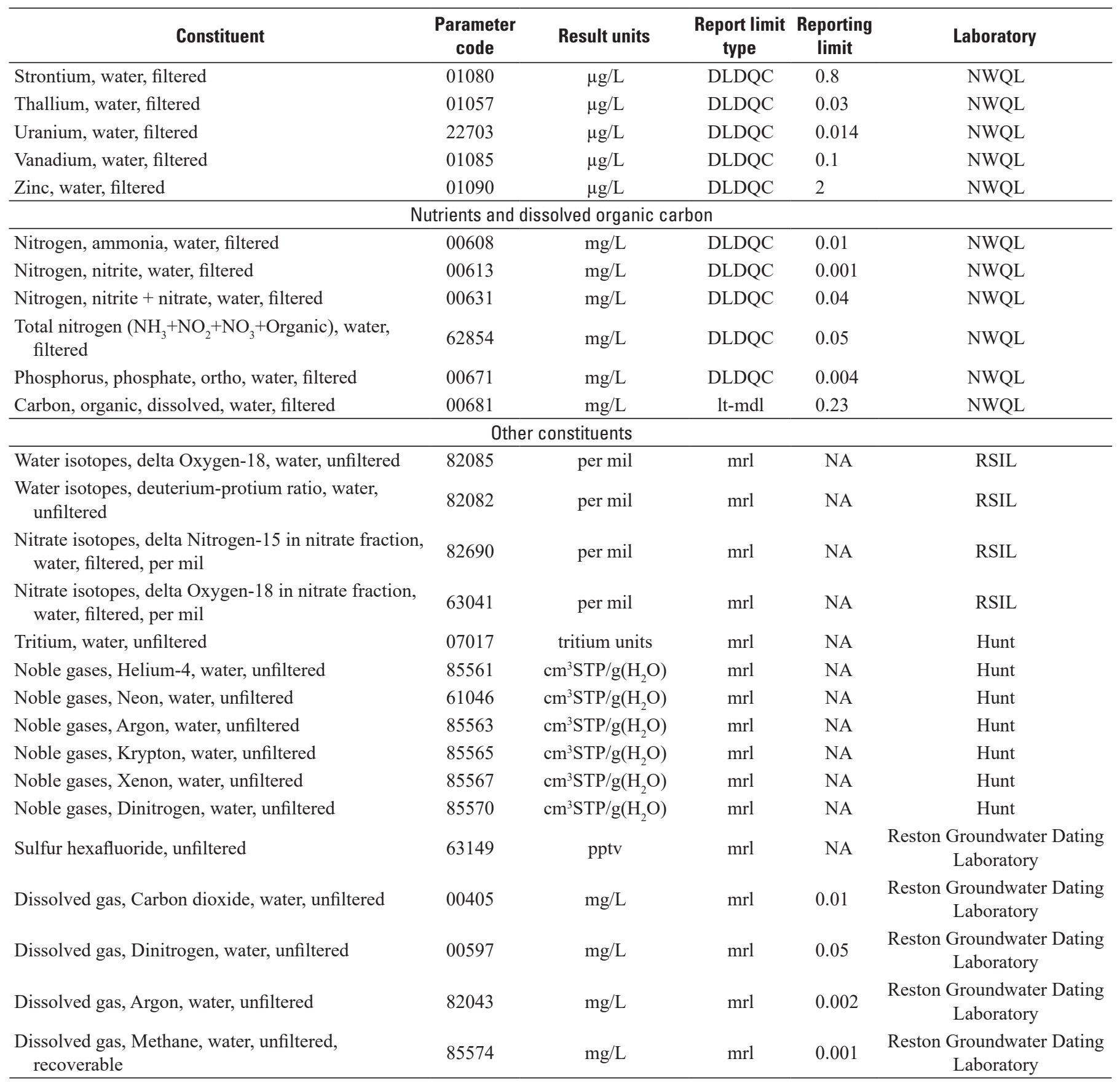

${ }^{1}$ Stored in National Water Information System as selenate calculated by subtracting selenite from selenium. 
contamination. Slight backpressure was applied to the tubes while filling to help keep the gases in solution. Samples for $\mathrm{SF}_{6}$ analysis were unfiltered and collected into 1-liter baked amber glass bottles with polyseal caps and then secured with electrical tape (USGS, 2015b). Samples for analysis of dissolved gases (carbon dioxide, dinitrogen, argon, and methane) were unfiltered and collected in glass bottles that were filled and capped with thick rubber stoppers under water to exclude headspace (atmospheric contamination) (USGS, 2015b).

Major ions, trace elements, nutrients, and dissolved organic carbon were analyzed by using standard methods (Brenton and Arnett, 1993; Garbarino and others, 2006; Fishman, 1993; Fishman and Friedman, 1989) at the USGS National Water Quality Laboratory (NWQL) in Lakewood, Colo. (table 3). Total selenium and selenite samples were analyzed by the USGS Colorado Water Science Center WaterQuality Research Lab (USGSCORL) in Denver, Colo., to a detection limit of $0.4 \mu \mathrm{g} / \mathrm{L}$ by using hydride generation-atomic fluorescence spectroscopy (HG-AFS) (Briggs and Crock, 1986) (table 3). An aliquot of the sample was diluted with hydrochloric acid, and the selenite concentration was measured by HG-AFS. A second aliquot of the sample was digested with a mixture of nitric, hydrochloric, and perchloric acid to convert all forms of selenium to selenite prior to analysis by HG-AFS (Briggs and Crock, 1986). Selenate was assumed to be the difference between total selenium and selenite, a similar approach to the one used by White and others (1991).

Water isotopes $\left(\delta^{2} \mathrm{H}-\mathrm{H}_{2} \mathrm{O}\right.$ and $\delta^{18} \mathrm{O}-\mathrm{H}_{2} \mathrm{O}$, ) were analyzed by the USGS Reston Stable Isotope Laboratory (RSIL) in Reston, Va., by using dual-inlet isotope-ratio mass spectrometry (Révész and Coplen, 2006). Hydrogen and oxygen isotopes in water are reported relative to Vienna standard mean ocean water (VSMOW). All isotope results are reported with the standard delta notation $(\delta)$, in per mil (\%o, parts per thousand) (table 3). For example, $\delta^{18} \mathrm{O}-\mathrm{H}_{2} \mathrm{O}$ is defined as

$$
\delta^{18} \mathrm{O}-\mathrm{H}_{2} \mathrm{O}=\left(\left({ }^{18} \mathrm{O} /{ }^{16} \mathrm{O}\right)_{\text {sample }} /\left({ }^{18} \mathrm{O} /{ }^{16} \mathrm{O}\right)_{\text {ref }}-1\right) \times 1,000
$$

where

${ }^{18} \mathrm{O} /{ }^{16} \mathrm{O}$ is the ratio of oxygen- 18 to oxygen- 16 in the sample and reference (ref) material (VSMOW in this example).

Samples of nitrate isotopes $\left(\delta^{15} \mathrm{~N}-\mathrm{NO}_{3}\right.$ and $\left.\delta^{18} \mathrm{O}-\mathrm{NO}_{3}\right)$ were only analyzed for selected wells where nitrate concentrations were 5 milligrams per liter $(\mathrm{mg} / \mathrm{L})$ or greater. The isotopic composition of nitrate $\left(\delta^{15} \mathrm{~N}-\mathrm{NO}_{3}\right.$ and $\left.\delta^{18} \mathrm{O}-\mathrm{NO}_{3}\right)$ was analyzed by bacterial conversion of nitrate to nitrous oxide and subsequent measurement on a continuous-flow isotoperatio mass spectrometer at the RSIL (Sigman and others, 2001; Casciotti and others, 2002; Coplen and others, 2004; Coplen and others, 2012). Oxygen isotopes in nitrate are reported relative to VSMOW, and nitrogen isotopes in nitrate are reported relative to atmospheric nitrogen gas $\left(\mathrm{N}_{2}\right)$.

Tritium analysis was performed at the USGS Noble Gas Laboratory in Denver, Colo., by using liquid scintillation counters; results are reported in tritium units (Hunt, 2015).
Concentrations of noble gases in cubic centimeters at standard temperature and pressure per gram of water $\left(\mathrm{cm}^{3} \mathrm{STP} / \mathrm{g}\left(\mathrm{H}_{2} \mathrm{O}\right)\right)$ and helium-3/helium-4 ratios relative to the helium-3/helium-4 ratio in air $\left(\mathrm{R} / \mathrm{R}_{\mathrm{a}}\right)$ were also measured at the USGS Noble Gas Laboratory (Hunt, 2015) (table 3).

Sulfur hexafluoride $\left(\mathrm{SF}_{6}\right)$ was measured at the USGS Reston Groundwater Dating Laboratory in Reston, Va., by using a purge-and-trap gas chromatography procedure with an electron-capture detector (USGS, 2015b) (table 3). Concentrations of dissolved gases (carbon dioxide, dinitrogen, argon, and methane, in milligrams per liter) were also measured at the USGS Reston Groundwater Dating Laboratory by using gas chromatography (USGS, 2015b).

\section{Data Analysis Methods}

Statistical tests used to evaluate water-quality data included the comparison of two groups and correlations between constituents. To compare two groups, the Wilcoxon rank-sum test (Helsel and Hirsch, 2002), which is a nonparametric test to evaluate two independent data groups, was used. Correlations between constituents were evaluated by using Pearson's $r$ correlation coefficient, also referred to as the linear correlation coefficient because $r$ measures the linear relationship between two variables (Helsel and Hirsch, 2002). Where $\mathrm{p}$ values were less than $0.01(\mathrm{p}<0.01)$, results were considered statistically significant. Summary statistics were used to present constituent concentrations, including minimum, maximum, and median concentrations. A trilinear diagram using the three-point plotting method, or Piper plot, was used to evaluate water types where data on major ion concentrations were converted into milliequivalents (Hem, 1985).

\section{Quality Assurance and Quality Control}

Quality-control samples, such as equipment blanks, field blanks, and replicate samples, were collected during each sampling event to evaluate potential contamination, bias, and variability in the data. The equipment blank was collected at the office to evaluate the potential for sample contamination from sampling equipment prior to any sampling. Field blanks were collected at four wells to evaluate potential sample contamination related to sample collection and to handling and equipment cleaning under field conditions (table 4). Field blanks for both the peristaltic pump and the submersible positive-pressure pump were processed using certified inorganic and organic blank waters. Replicate samples were collected to evaluate sampling and analytical variability. Four replicate samples were collected immediately after environmental samples to assess analytical variability and variability resulting from sample collection (table 5).

Blanks were used to evaluate potential contamination introduced to environmental samples during collection, processing, shipment, and laboratory analysis (Mueller and others, 2015). The following constituents were detected in equipment and (or) field blanks: ammonia, calcium, sodium, 
Table 4. Results of sample-blank analyses for study period.

$\left[\mathrm{mg} / \mathrm{L}\right.$, milligram per liter; $\mu \mathrm{g} / \mathrm{L}$, microgram per liter; <, less than; --, no data, ${ }^{\circ} \mathrm{C}$, degrees Celsius. Sample time given in 24 -hour format (HHMM). Values in bold format in maximum blank concentration row indicate detections in the blank. Gray shading indicates minimum environmental concentration less than 10 times the blank concentration]

\begin{tabular}{|c|c|c|c|c|c|c|c|c|c|}
\hline $\begin{array}{l}\text { Well identifier } \\
\text { (blank type) }\end{array}$ & $\begin{array}{l}\text { Sample date } \\
\text { and time }\end{array}$ & $\begin{array}{l}\text { Ammonia, } \\
\text { filtered } \\
\text { (mg/L) }\end{array}$ & $\begin{array}{l}\text { Nitrite, } \\
\text { filtered } \\
\text { (mg/L) }\end{array}$ & $\begin{array}{l}\text { Nitrate } \\
\text { plus nitrite, } \\
\text { filtered } \\
\text { (mg/L) }\end{array}$ & $\begin{array}{c}\text { Ortho- } \\
\text { phosphate, } \\
\text { filtered } \\
\text { (mg/L) }\end{array}$ & $\begin{array}{c}\text { Organic } \\
\text { carbon, } \\
\text { filtered } \\
\text { (mg/L) }\end{array}$ & $\begin{array}{l}\text { Calcium, } \\
\text { filtered } \\
\text { (mg/L) }\end{array}$ & $\begin{array}{c}\text { Magnesium, } \\
\text { filtered } \\
\text { (mg/L) }\end{array}$ & $\begin{array}{l}\text { Sodium, } \\
\text { filtered } \\
\text { (mg/L) }\end{array}$ \\
\hline 16 (equipment blank) & $\begin{array}{c}\text { 7/9/2014 } \\
0959\end{array}$ & 0.029 & $<0.001$ & $<0.04$ & $<0.004$ & $<0.23$ & $<0.022$ & $<0.011$ & $<0.06$ \\
\hline $\begin{array}{l}14 \text { (field blank sub- } \\
\text { mersible pump) }\end{array}$ & $\begin{array}{l}3 / 4 / 2015 \\
1429\end{array}$ & 0.031 & $<0.001$ & $<0.04$ & $<0.004$ & $<0.23$ & 0.055 & $<0.011$ & 0.104 \\
\hline $\begin{array}{r}6 \text { (field blank sub- } \\
\text { mersible pump) }\end{array}$ & $\begin{array}{c}3 / 12 / 2015 \\
0759\end{array}$ & $<0.01$ & $<0.001$ & $<0.04$ & $<0.004$ & $<0.23$ & 0.079 & $<0.011$ & $<0.06$ \\
\hline $\begin{array}{l}\text { Maximum blank } \\
\text { concentration }\end{array}$ & & 0.031 & $<0.001$ & $<0.04$ & $<0.004$ & $<0.23$ & 0.079 & $<0.011$ & 0.104 \\
\hline $\begin{array}{l}\text { Minimum environ- } \\
\text { mental sample } \\
\text { concentration }\end{array}$ & & $<0.01$ & 0.001 & 0.054 & 0.004 & 1.8 & 170 & 42 & 62 \\
\hline $\begin{array}{l}\text { Maximum environ- } \\
\text { mental sample } \\
\text { concentration }\end{array}$ & & 19 & 7.1 & 550 & 0.18 & 83 & 617 & 2,500 & 13,000 \\
\hline $\begin{array}{l}\text { Well identifier } \\
\text { (blank type) }\end{array}$ & $\begin{array}{l}\text { Potassium, } \\
\text { filtered } \\
\text { (mg/L) }\end{array}$ & $\begin{array}{l}\text { Chloride, } \\
\text { filtered } \\
\text { (mg/L) }\end{array}$ & $\begin{array}{l}\text { Sulfate, } \\
\text { filtered } \\
\text { (mg/L) }\end{array}$ & $\begin{array}{c}\text { Fluoride, } \\
\text { filtered } \\
\text { (mg/L) }\end{array}$ & $\begin{array}{c}\text { Silica, } \\
\text { filtered } \\
\text { (mg/L) }\end{array}$ & $\begin{array}{c}\text { Arsenic, } \\
\text { filtered } \\
\text { ( } \mu \mathrm{g} / \mathrm{L})\end{array}$ & $\begin{array}{c}\text { Barium, } \\
\text { filtered } \\
\text { ( } \mu \mathrm{g} / \mathrm{L})\end{array}$ & $\begin{array}{c}\text { Beryllium, } \\
\text { filtered } \\
(\mu \mathrm{g} / \mathrm{L})\end{array}$ & $\begin{array}{c}\text { Boron, } \\
\text { filtered } \\
\text { ( } \mu \mathrm{g} / \mathrm{L})\end{array}$ \\
\hline $\begin{array}{l}14 \text { (field blank sub- } \\
\text { mersible pump) }\end{array}$ & $<0.03$ & $<0.02$ & 0.113 & 0.018 & 0.104 & $<0.1$ & $<0.25$ & $<0.02$ & $<5$ \\
\hline $\begin{array}{l}6 \text { (field blank sub- } \\
\text { mersible pump) }\end{array}$ & $<0.03$ & $<0.02$ & 0.04 & $<0.01$ & $<0.018$ & $<0.1$ & $<0.25$ & $<0.02$ & $<5$ \\
\hline Reporting limit & 0.03 & $0.02,0.06$ & $0.02,0.09$ & 0.01 & 0.018 & $0.04,0.1$ & $0.1,0.25$ & $0.006,0.02$ & 3,5 \\
\hline $\begin{array}{r}\text { Minimum blank } \\
\text { concentration }\end{array}$ & $<0.03$ & $<0.02$ & $<0.02$ & $<0.01$ & $<0.018$ & $<0.04$ & $<0.1$ & $<0.006$ & $<3$ \\
\hline $\begin{array}{c}\text { Maximum blank } \\
\text { concentration }\end{array}$ & $<0.03$ & $<0.02$ & 0.113 & 0.018 & 0.104 & $<0.1$ & $<0.25$ & $<0.02$ & $<5$ \\
\hline $\begin{array}{l}\text { Minimum environ- } \\
\text { mental sample } \\
\text { concentration }\end{array}$ & 1.8 & 0.91 & 530 & 0.17 & 6.0 & 0.22 & 6.5 & 0.008 & 120 \\
\hline $\begin{array}{l}\text { Maximum environ- } \\
\text { mental sample }\end{array}$ & 53 & 3,900 & 34,000 & 1.70 & 32 & 8.00 & 46 & 0.075 & 1,600 \\
\hline
\end{tabular}


Table 4. Results of sample-blank analyses for study period.-Continued

$\left[\mathrm{mg} / \mathrm{L}\right.$, milligram per liter; $\mu \mathrm{g} / \mathrm{L}$, microgram per liter; <, less than; --, no data, ${ }^{\circ} \mathrm{C}$, degrees Celsius. Sample time given in 24-hour format (HHMM). Values in bold format in maximum blank concentration row indicate detections in the blank. Gray shading indicates minimum environmental concentration less than 10 times the blank concentration]

\begin{tabular}{|c|c|c|c|c|c|c|c|c|c|}
\hline $\begin{array}{l}\text { Well identifier } \\
\text { (blank type) }\end{array}$ & $\begin{array}{c}\text { Cadmium, } \\
\text { filtered } \\
\text { ( } \mu \mathrm{g} / \mathrm{L})\end{array}$ & $\begin{array}{c}\text { Chromium, } \\
\text { filtered } \\
(\mu \mathrm{g} / \mathrm{L})\end{array}$ & $\begin{array}{c}\text { Cobalt, } \\
\text { filtered } \\
\text { ( } \mu \mathrm{g} / \mathrm{L})\end{array}$ & $\begin{array}{c}\text { Copper, } \\
\text { filtered } \\
\text { ( } \mu \mathrm{g} / \mathrm{L})\end{array}$ & $\begin{array}{c}\text { Iron, } \\
\text { filtered } \\
\text { ( } \mu \mathrm{g} / \mathrm{L} \text { ) }\end{array}$ & $\begin{array}{c}\text { Lead, } \\
\text { filtered } \\
\text { ( } \mu \mathrm{g} / \mathrm{L})\end{array}$ & $\begin{array}{c}\text { Man- } \\
\text { ganese, } \\
\text { filtered } \\
(\mu \mathrm{g} / \mathrm{L})\end{array}$ & $\begin{array}{l}\text { Thallium, } \\
\text { filtered } \\
\text { ( } \mu \mathrm{g} / \mathrm{L})\end{array}$ & $\begin{array}{l}\text { Molybde- } \\
\text { num, } \\
\text { filtered } \\
\text { ( } \mu \mathrm{g} / \mathrm{L})\end{array}$ \\
\hline $\begin{array}{l}15 \text { (field blank using } \\
\text { peristaltic pump) }\end{array}$ & $<0.016$ & $<0.07$ & $<0.023$ & $<0.8$ & $<4$ & $<0.025$ & $<0.15$ & $<0.01$ & $<0.014$ \\
\hline 16 (equipment blank) & $<0.03$ & $<0.3$ & $<0.05$ & $<0.8$ & $<4$ & $<0.04$ & $<0.4$ & $<0.03$ & 0.51 \\
\hline $\begin{array}{c}14 \text { (field blank sub- } \\
\text { mersible pump) }\end{array}$ & $<0.03$ & $<0.3$ & $<0.05$ & 1.8 & $<4$ & $<0.04$ & $<0.4$ & $<0.03$ & 0.071 \\
\hline $\begin{array}{l}6 \text { (field blank sub- } \\
\text { mersible pump) }\end{array}$ & $<0.03$ & $<0.3$ & $<0.05$ & 1.1 & $<4$ & $<0.04$ & $<0.4$ & $<0.03$ & 0.078 \\
\hline $\begin{array}{c}\text { Maximum blank } \\
\text { concentration }\end{array}$ & $<0.03$ & $<0.3$ & $<0.023$ & 1.8 & $<4$ & $<0.025$ & $<0.15$ & $<0.01$ & 0.51 \\
\hline $\begin{array}{l}\text { Minimum environ- } \\
\text { mental sample } \\
\text { concentration }\end{array}$ & 0.041 & $<0.3$ & 0.13 & 1.4 & 5.3 & 0.12 & 3.7 & 0.012 & 1.3 \\
\hline $\begin{array}{l}\text { Maximum environ- } \\
\text { mental sample } \\
\text { concentration }\end{array}$ & 1.2 & $<0.3$ & 26 & 32 & 8,650 & 3.6 & 1,400 & 11 & 130 \\
\hline $\begin{array}{l}\text { Well identifier } \\
\text { (blank type) }\end{array}$ & $\begin{array}{c}\text { Nickel, } \\
\text { filtered } \\
\text { ( } \mu \mathrm{g} / \mathrm{L})\end{array}$ & $\begin{array}{c}\text { Silver, } \\
\text { filtered } \\
\text { ( } \mu \mathrm{g} / \mathrm{L})\end{array}$ & $\begin{array}{c}\text { Strontium, } \\
\text { filtered } \\
(\mu \mathrm{g} / \mathrm{L})\end{array}$ & $\begin{array}{c}\text { Vanadium, } \\
\text { filtered } \\
(\mu \mathrm{g} / \mathrm{L})\end{array}$ & $\begin{array}{c}\text { Zinc, } \\
\text { filtered } \\
(\mu \mathrm{g} / \mathrm{L})\end{array}$ & $\begin{array}{l}\text { Antimony, } \\
\text { filtered } \\
(\mu \mathrm{g} / \mathrm{L})\end{array}$ & $\begin{array}{c}\text { Aluminum, } \\
\text { filtered } \\
(\mu \mathrm{g} / \mathrm{L})\end{array}$ & $\begin{array}{l}\text { Lithium, } \\
\text { filtered } \\
\text { ( } \mu \mathrm{g} / \mathrm{L} \text { ) }\end{array}$ & $\begin{array}{c}\text { Selenium, } \\
\text { filtered } \\
(\mu \mathrm{g} / \mathrm{L})\end{array}$ \\
\hline $\begin{array}{l}14 \text { (field blank sub- } \\
\text { mersible pump) }\end{array}$ & $<0.2$ & 0.026 & $<0.8$ & $<0.1$ & $<2$ & $<0.027$ & $<3$ & $<0.22$ & $<0.05$ \\
\hline $\begin{array}{r}6 \text { (field blank sub- } \\
\text { mersible pump) }\end{array}$ & 0.32 & $<0.02$ & 1.00 & $<0.1$ & $<2$ & $<0.027$ & $<3$ & $<0.22$ & $<0.05$ \\
\hline Reporting limit & $0.09,0.2$ & $0.005,0.02$ & $0.2,0.8$ & $0.08,0.1$ & $1.4,2$ & 0.027 & $2.2,3$ & 0.22 & $0.03,0.05$ \\
\hline $\begin{array}{r}\text { Minimum blank } \\
\text { concentration }\end{array}$ & $<0.09$ & $<0.005$ & $<0.2$ & $<0.08$ & $<1.4$ & $<0.027$ & $<2.2$ & $<0.22$ & $<0.03$ \\
\hline $\begin{array}{l}\text { Maximum blank } \\
\text { concentration }\end{array}$ & 0.32 & 0.026 & 1.00 & $<0.08$ & $<1.4$ & $<0.027$ & $<2.2$ & $<0.22$ & $<0.03$ \\
\hline $\begin{array}{l}\text { Minimum environ- } \\
\text { mental sample } \\
\text { concentration }\end{array}$ & 1.4 & 0.012 & 1,300 & 0.32 & 2.9 & 0.056 & 6.3 & 75 & 0.16 \\
\hline $\begin{array}{l}\text { Maximum environ- } \\
\text { mental sample } \\
\text { concentration }\end{array}$ & 160 & 1.2 & 15,000 & 9.1 & 20 & 0.62 & 6.3 & 4,900 & 4,100 \\
\hline
\end{tabular}


Table 4. Results of sample-blank analyses for study period.-Continued

$\left[\mathrm{mg} / \mathrm{L}\right.$, milligram per liter; $\mu \mathrm{g} / \mathrm{L}$, microgram per liter; <, less than; --, no data, ${ }^{\circ} \mathrm{C}$, degrees Celsius. Sample time given in 24-hour format (HHMM). Values in bold format in maximum blank concentration row indicate detections in the blank. Gray shading indicates minimum environmental concentration less than 10 times the blank concentration]

\begin{tabular}{|c|c|c|c|c|c|c|}
\hline $\begin{array}{l}\text { Well identifier } \\
\text { (blank type) }\end{array}$ & $\begin{array}{l}\text { Uranium, } \\
\text { filtered } \\
\text { ( } \mu \mathrm{g} / \mathrm{L})\end{array}$ & $\begin{array}{c}\text { Total } \\
\text { nitrogen, } \\
\text { filtered } \\
\text { (mg/L) }\end{array}$ & $\begin{array}{l}\text { Selenate, } \\
\text { filtered } \\
(\mu \mathrm{g} / \mathrm{L})\end{array}$ & $\begin{array}{l}\text { Selenite, } \\
\text { filtered } \\
\text { ( } \mu \mathrm{g} / \mathrm{L})\end{array}$ & $\begin{array}{l}\text { Dissolved } \\
\text { solids, dry } \\
\text { at } 180^{\circ} \mathrm{C} \\
\text { (mg/L) }\end{array}$ & $\begin{array}{c}\text { Bromide, } \\
\text { filtered } \\
\text { (mg/L) }\end{array}$ \\
\hline $\begin{array}{l}15 \text { (field blank using } \\
\text { peristaltic pump) }\end{array}$ & $<0.004$ & $<0.05$ & -- & -- & $<20$ & $<0.01$ \\
\hline 16 (equipment blank) & $<0.014$ & $<0.05$ & -- & -- & $<20$ & $<0.03$ \\
\hline $\begin{array}{l}27 \text { (field blank sub- } \\
\text { mersible pump) }\end{array}$ & -- & -- & $<0.4$ & $<0.4$ & -- & -- \\
\hline $\begin{array}{l}14 \text { (field blank sub- } \\
\text { mersible pump) }\end{array}$ & $<0.014$ & $<0.05$ & -- & -- & $<20$ & $<0.03$ \\
\hline $\begin{array}{l}6 \text { (field blank sub- } \\
\text { mersible pump) }\end{array}$ & $<0.014$ & $<0.05$ & -- & -- & $<20$ & $<0.03$ \\
\hline Reporting limit & $0.004,0.014$ & 0.05 & 0.4 & 0.4 & 20 & $0.01,0.03$ \\
\hline $\begin{array}{r}\text { Minimum blank } \\
\text { concentration }\end{array}$ & $<0.004$ & $<0.05$ & $<0.4$ & $<0.4$ & $<20$ & $<0.01$ \\
\hline $\begin{array}{c}\text { Maximum blank } \\
\text { concentration }\end{array}$ & $<0.004$ & $<0.05$ & $<0.4$ & $<0.4$ & $<20$ & $<0.01$ \\
\hline $\begin{array}{l}\text { Minimum environ- } \\
\text { mental sample } \\
\text { concentration }\end{array}$ & 10 & 0.12 & 0.44 & 0.45 & 930 & 0.078 \\
\hline $\begin{array}{l}\text { Maximum environ- } \\
\text { mental sample } \\
\text { concentration }\end{array}$ & 470 & 520 & 3,000 & 15 & 53,000 & 31 \\
\hline
\end{tabular}

sulfate, fluoride, silica, copper, molybdenum, nickel, silver, and strontium (table 4). Environmental concentrations greater than 10 times the amount detected in the blank were considered valid results (Mueller and others, 2015). Environmental concentrations were less than 10 times the blank concentration for several constituents including ammonia, fluoride, copper, molybdenum, nickel, and silver. The following number of results were affected by these blank detections: 66 ammonia, 1 fluoride, 66 copper, 24 molybdenum, 4 nickel, and 69 silver. Therefore, environmental results for ammonia, copper, molybdenum, nickel, and silver were not included in analysis and interpretations because of blank contamination. The single fluoride result affected would not change the interpretations presented in this report.

Replicate samples were used to estimate variability of analytical results by calculating the relative percent difference (RPD) between replicate pairs (table 5); RPDs greater than 20 percent indicate that high variability might affect interpretation of the environmental data (Mueller and others, 2015). The following constituents had RPDs greater than 20 percent: ammonia, orthophosphate, fluoride, barium, boron, cobalt, lead, manganese, thallium, molybdenum, nickel, strontium, vanadium, selenium, and uranium (table 5). High RPDs associated with the replicate pair at well 8 on September 3, 2013, account for the majority of the RPDs greater than 20 percent and appear to be associated primarily with the trace element results for this replicate pair. This replicate pair had RPDs greater than 20 percent for barium, boron, cobalt, manganese, thallium, molybdenum, nickel, strontium, vanadium, selenium, and uranium (table 5). Overall, the results from this single replicate pair at well 8 would not change the interpretations presented in this report. Where concentrations in replicate pairs were near the detection limit, RPDs greater than 20 percent were not considered to influence analysis and interpretation, which is the case for ammonia, orthophosphate, fluoride, lead, and thallium.

Ion balance was used to evaluate sample quality by determining the difference (in milliequivalents) between cations (calcium, magnesium, sodium, potassium, iron, and manganese) and anions (bicarbonate, chloride, fluoride, sulfate, and nitrate) for each environmental sample. Ion balances were calculated according to methods in Hem (1985). Ion balances for environmental samples were all found to be less than 10 percent. Ten percent is considered an acceptable limit for charge balance because groundwater may contain additional constituents that are participating in the balance, such as organic anions that form complexes with metals. In these cases, analytical procedures will not give results that 
Table 5. Results of sample-replicate analyses for study period.

$\left[\mathrm{mg} / \mathrm{L}\right.$, milligram per liter; $\mu \mathrm{g} / \mathrm{L}$, microgram per liter; $<$, less than; --, no data; ${ }^{\circ} \mathrm{C}$ degrees Celsius, relative percent difference, the absolute difference between replicate analyses divided by the average of the analyses and expressed as percent (values in bold format). Sample time given in 24-hour format (HHMM)]

\begin{tabular}{|c|c|c|c|c|c|c|c|c|c|}
\hline $\begin{array}{c}\text { Well } \\
\text { identifier }\end{array}$ & $\begin{array}{l}\text { Sample date } \\
\text { and time }\end{array}$ & $\begin{array}{l}\text { Sample } \\
\text { type }\end{array}$ & $\begin{array}{c}\text { Ammonia, } \\
\text { filtered } \\
\text { (mg/L) }\end{array}$ & $\begin{array}{l}\text { Nitrite, } \\
\text { filtered } \\
(\mathrm{mg} / \mathrm{L})\end{array}$ & $\begin{array}{l}\text { Nitrate } \\
\text { plus } \\
\text { nitrite, } \\
\text { filtered } \\
\text { (mg/L) }\end{array}$ & $\begin{array}{c}\text { Ortho- } \\
\text { phosphate, } \\
\text { filtered } \\
\text { (mg/L) }\end{array}$ & $\begin{array}{c}\text { Organic } \\
\text { carbon, } \\
\text { filtered } \\
\text { (mg/L) }\end{array}$ & $\begin{array}{c}\text { Calcium, } \\
\text { filtered } \\
\text { (mg/L) }\end{array}$ & \\
\hline 8 & 9/3/2013, 1045 & environmental & 0.20 & 0.40 & 38 & 0.021 & -- & 426 & \\
\hline 8 & 9/3/2013, 1046 & replicate & 0.24 & 0.40 & 37 & 0.020 & -- & 426 & \\
\hline 6 & $8 / 6 / 2014,1531$ & replicate & $<0.01$ & 0.002 & 5.2 & 0.008 & 3.0 & 316 & \\
\hline Relative percent difference & & & -- & 4.0 & 0.7 & 0.4 & 1.1 & 9.3 & \\
\hline 10 & $3 / 11 / 2015,1400$ & environmental & 0.056 & 0.055 & 0.13 & 0.005 & 3.9 & 493 & \\
\hline 10 & $3 / 11 / 2015,1401$ & replicate & 0.041 & 0.053 & 0.12 & 0.006 & 4.5 & 479 & \\
\hline 22 & $3 / 4 / 2015,1101$ & replicate & 0.18 & 0.83 & 46 & 0.024 & 26 & 431 & \\
\hline Relative percent difference & & & 16.0 & 0.1 & 4.0 & 4.5 & 0.0 & 4.0 & \\
\hline Minimum relative percent difference & & & -- & 0.1 & 0.7 & 0.4 & 0.0 & 0.0 & \\
\hline Maximum relative percent difference & & & 31.4 & 4.6 & 8.5 & 27.4 & 15.7 & 9.3 & \\
\hline $\begin{array}{c}\text { Well } \\
\text { identifier }\end{array}$ & $\begin{array}{l}\text { Magnesium, } \\
\text { filtered } \\
(\mathrm{mg} / \mathrm{L})\end{array}$ & $\begin{array}{c}\text { Sodium, } \\
\text { filtered } \\
\text { (mg/L) }\end{array}$ & $\begin{array}{c}\text { Potassium, } \\
\text { filtered } \\
\text { (mg/L) }\end{array}$ & $\begin{array}{c}\text { Chloride, } \\
\text { filtered } \\
\text { (mg/L) }\end{array}$ & $\begin{array}{c}\text { Sulfate, } \\
\text { filtered } \\
\text { (mg/L) }\end{array}$ & $\begin{array}{c}\text { Fluoride, } \\
\text { filtered } \\
\text { (mg/L) }\end{array}$ & $\begin{array}{l}\text { Silica, } \\
\text { filtered } \\
(\mathrm{mg} / \mathrm{L})\end{array}$ & $\begin{array}{c}\text { Arsenic, } \\
\text { filtered } \\
(\mu \mathrm{g} / \mathrm{L})\end{array}$ & $\begin{array}{c}\text { Barium, } \\
\text { filtered } \\
(\mu \mathrm{g} / \mathrm{L})\end{array}$ \\
\hline 8 & 464 & 1,652 & 13.17 & 124 & 5,487 & 0.467 & 14.86 & $<0.24$ & 8.16 \\
\hline 8 & 461 & 1,644 & 12.93 & 120 & 5,435 & 0.426 & 14.94 & 0.309 & 10.2 \\
\hline 10 & 177 & 409 & 14 & 7.8 & 2,118 & 0.35 & 9.2 & 1.8 & 9.68 \\
\hline Relative percent difference & 2.1 & 0.1 & 5.9 & 4.1 & 2.9 & 3.7 & 1.6 & 8.3 & 0.9 \\
\hline 22 & 588 & 1,721 & 14.9 & 186 & 5,237 & 0.538 & 15.7 & 1.99 & 8.97 \\
\hline 22 & 611 & 1,681 & 18.0 & 175 & 4,911 & 0.624 & 15.9 & 2.03 & 9.26 \\
\hline Relative percent difference & 3.9 & 2.4 & 18.5 & 5.8 & 6.4 & 14.8 & 1.2 & 2.0 & 3.2 \\
\hline Minimum relative percent difference & 0.8 & 0.1 & 1.8 & 3.4 & 1.0 & 3.7 & 0.5 & -- & 0.9 \\
\hline Maximum relative percent difference & 3.9 & 2.4 & 18.5 & 12.3 & 13.6 & 24.9 & 1.6 & 8.3 & 22.2 \\
\hline
\end{tabular}


Table 5. Results of sample-replicate analyses for study period.-Continued

$\left[\mathrm{mg} / \mathrm{L}\right.$, milligram per liter; $\mu \mathrm{g} / \mathrm{L}$, microgram per liter; $<$, less than; --, no data; ${ }^{\circ} \mathrm{C}$ degrees Celsius, relative percent difference, the absolute difference between replicate analyses divided by the average of the analyses and expressed as percent (values in bold format). Sample time given in 24-hour format (HHMM)]

\begin{tabular}{|c|c|c|c|c|c|c|c|c|c|}
\hline $\begin{array}{c}\text { Well } \\
\text { identifier }\end{array}$ & $\begin{array}{c}\text { Beryllium, } \\
\text { filtered } \\
(\mu \mathrm{g} / \mathrm{L})\end{array}$ & $\begin{array}{c}\text { Boron, } \\
\text { filtered } \\
(\mu \mathrm{g} / \mathrm{L})\end{array}$ & $\begin{array}{c}\text { Cadmium, } \\
\text { filtered } \\
(\mu \mathrm{g} / \mathrm{L})\end{array}$ & $\begin{array}{c}\text { Chro- } \\
\text { mium, } \\
\text { filtered } \\
(\mu \mathrm{g} / \mathrm{L})\end{array}$ & $\begin{array}{l}\text { Cobalt, } \\
\text { filtered } \\
(\mu \mathrm{g} / \mathrm{L})\end{array}$ & $\begin{array}{c}\text { Copper, } \\
\text { filtered } \\
(\mu \mathrm{g} / \mathrm{L})\end{array}$ & $\begin{array}{l}\text { Iron, } \\
\text { filtered } \\
(\mu \mathrm{g} / \mathrm{L})\end{array}$ & $\begin{array}{l}\text { Lead, } \\
\text { filtered } \\
(\mu \mathrm{g} / \mathrm{L})\end{array}$ & $\begin{array}{c}\text { Manganese, } \\
\text { filtered } \\
(\mu \mathrm{g} / \mathrm{L})\end{array}$ \\
\hline 8 & $<0.042$ & 1,069 & $<0.112$ & $<0.49$ & 4.10 & $<5.6$ & $<24$ & $<0.175$ & 981 \\
\hline Relative percent difference & -- & 21.5 & -- & -- & 25.4 & -- & -- & -- & 23.1 \\
\hline 6 & $<0.02$ & 251 & -- & $<0.3$ & 1.32 & 2.937 & 9.01 & 0.044 & 4.34 \\
\hline Relative percent difference & -- & 15.3 & -- & -- & 7.2 & -- & -- & 166.1 & 15.3 \\
\hline 10 & $<0.2$ & 789 & $<0.3$ & $<3$ & 1.37 & $<8$ & 36.9 & $<0.4$ & 222 \\
\hline 10 & $<0.2$ & 801 & $<0.3$ & $<3$ & 1.31 & $<8$ & 33.0 & $<0.4$ & 219 \\
\hline Relative percent difference & -- & 2.2 & -- & -- & 3.1 & -- & -- & -- & 1.0 \\
\hline Minimum relative percent difference & -- & 1.4 & -- & -- & 3.1 & -- & -- & -- & 1.0 \\
\hline Maximum relative percent difference & -- & 21.5 & -- & -- & 25.4 & -- & 11.1 & 166.1 & 23.1 \\
\hline $\begin{array}{c}\text { Well } \\
\text { identifier }\end{array}$ & $\begin{array}{c}\text { Thallium, } \\
\text { filtered } \\
\text { ( } \mu \mathrm{g} / \mathrm{L})\end{array}$ & $\begin{array}{l}\text { Molybdenum, } \\
\text { filtered } \\
(\mu \mathrm{g} / \mathrm{L})\end{array}$ & $\begin{array}{c}\text { Nickel, } \\
\text { filtered } \\
(\mu \mathrm{g} / \mathrm{L})\end{array}$ & $\begin{array}{c}\text { Silver, } \\
\text { filtered } \\
(\mu \mathrm{g} / \mathrm{L})\end{array}$ & $\begin{array}{c}\text { Strontium, } \\
\text { filtered } \\
(\mu \mathrm{g} / \mathrm{L})\end{array}$ & $\begin{array}{c}\text { Vanadium, } \\
\text { filtered } \\
(\mu \mathrm{g} / \mathrm{L})\end{array}$ & $\begin{array}{c}\text { Zinc, } \\
\text { filtered } \\
(\mu \mathrm{g} / \mathrm{L})\end{array}$ & $\begin{array}{c}\text { Antimony, } \\
\text { filtered } \\
(\mu \mathrm{g} / \mathrm{L})\end{array}$ & $\begin{array}{c}\text { Aluminum, } \\
\text { filtered } \\
(\mu \mathrm{g} / \mathrm{L})\end{array}$ \\
\hline 8 & 0.100 & 9.85 & 19.8 & $<0.03$ & 7,074 & 0.687 & $<8.4$ & $<0.162$ & $<13.2$ \\
\hline 8 & 0.128 & 12.5 & 25.9 & $<0.035$ & 8,939 & 0.860 & $<9.8$ & $<0.189$ & $<15.4$ \\
\hline Relative percent difference & -- & 2.3 & 3.0 & -- & 0.1 & 21.7 & -- & -- & -- \\
\hline 22 & 0.416 & 20.1 & 56.3 & $<0.2$ & 8,340 & 1.48 & $<20$ & $<0.27$ & $<30$ \\
\hline 22 & 0.444 & 20.2 & 57.4 & $<0.2$ & 8,474 & 1.47 & $<20$ & $<0.27$ & $<30$ \\
\hline Relative percent difference & 6.5 & 0.4 & 1.9 & -- & 1.6 & 0.4 & -- & -- & -- \\
\hline Minimum relative percent difference & -- & 0.4 & 0.0 & -- & 0.1 & 0.4 & -- & -- & -- \\
\hline Maximum relative percent difference & 25.0 & 23.4 & 26.9 & -- & 23.3 & 22.3 & -- & 2.7 & -- \\
\hline
\end{tabular}


Table 5. Results of sample-replicate analyses for study period.—Continued

$\left[\mathrm{mg} / \mathrm{L}\right.$, milligram per liter; $\mu \mathrm{g} / \mathrm{L}$, microgram per liter; $<$, less than; --, no data; ${ }^{\circ} \mathrm{C}$ degrees Celsius, relative percent difference, the absolute difference between replicate analyses divided by the average of the analyses and expressed as percent (values in bold format). Sample time given in 24-hour format (HHMM)]

\begin{tabular}{|c|c|c|c|c|c|c|c|c|}
\hline $\begin{array}{c}\text { Well } \\
\text { identifier }\end{array}$ & $\begin{array}{c}\text { Lithium, } \\
\text { filtered } \\
\text { ( } \mu \mathrm{g} / \mathrm{L})\end{array}$ & $\begin{array}{c}\text { Selenium, } \\
\text { filtered } \\
\text { ( } \mu \mathrm{g} / \mathrm{L})\end{array}$ & $\begin{array}{c}\text { Uranium, } \\
\text { filtered } \\
\text { ( } \mu \mathrm{g} / \mathrm{L})\end{array}$ & $\begin{array}{c}\text { Total } \\
\text { nitrogen, } \\
\text { filtered } \\
\text { (mg/L) }\end{array}$ & $\begin{array}{c}\text { Selenate, } \\
\text { filtered } \\
(\mu \mathrm{g} / \mathrm{L})\end{array}$ & $\begin{array}{c}\text { Selenite, } \\
\text { filtered } \\
(\mu \mathrm{g} / \mathrm{L})\end{array}$ & $\begin{array}{c}\text { Dissolved } \\
\text { solids, } \\
\text { dry at } \\
180^{\circ} \mathrm{C} \\
\text { (mg/L) }\end{array}$ & $\begin{array}{c}\text { Bromide, } \\
\text { filtered } \\
\text { (mg/L) }\end{array}$ \\
\hline 8 & 573 & 530 & 89.8 & 36.0 & 558 & 0.86 & 10,186 & $<1$ \\
\hline 8 & 678 & 718 & 114 & 37.2 & 530 & 1.00 & 10,076 & $<1$ \\
\hline Relative percent difference & 16.9 & 30.1 & 23.5 & 3.4 & 5.1 & 15.1 & 1.1 & -- \\
\hline 6 & 121 & 23 & 14.95 & 5.34 & 26 & $<0.4$ & 1,772 & $<0.15$ \\
\hline 6 & 121 & 25 & 16.75 & 5.211 & 24 & $<0.4$ & 1,782 & 0.074 \\
\hline Relative percent difference & 0.3 & 5.0 & 11.4 & 2.4 & 8.0 & -- & 0.6 & -- \\
\hline 10 & 380 & 0.703 & 17.3 & 0.331 & 0.68 & $<0.4$ & 4,080 & $<0.6$ \\
\hline 10 & 388 & 1.031 & 18.5 & 0.322 & -- & -- & 4,108 & $<0.6$ \\
\hline Relative percent difference & 2.1 & 37.8 & 6.6 & 2.8 & -- & -- & 0.7 & -- \\
\hline 22 & 552 & 1,141 & 137 & 47.855 & 1,292 & 5.63 & 10,088 & $<1.5$ \\
\hline 22 & 565 & 1,155 & 140 & 48.642 & 1,257 & 5.24 & 10,016 & $<1.5$ \\
\hline Relative percent difference & 2.3 & 1.2 & 2.1 & 1.6 & 2.7 & 7.2 & 0.7 & -- \\
\hline Minimum relative percent difference & 0.3 & 1.2 & 2.1 & 1.6 & -- & -- & 0.6 & -- \\
\hline Maximum relative percent difference & 16.9 & 37.8 & 23.5 & 3.4 & 8.0 & 15.1 & 1.1 & -- \\
\hline
\end{tabular}

can be balanced satisfactorily (Hem, 1985). Several wells had yellow to orange discharge, which may be an indication of the presence of organic anions that form complexes with metals. If that is the case, the usual analytical procedures may not provide accurate measurement of these anions (Hem, 1985).

As part of this study, the concentration of dissolved selenium was determined by two laboratories who use different methods, the NWQL and the USGSCORL. When the selenium concentrations from the two laboratories differed by an order of magnitude for the same sample, both values were rerun. A significant bias was observed in the data for selenium concentrations less than $20 \mu \mathrm{g} / \mathrm{L}(\mathrm{p}<0.01)$, with the NWQL reporting higher concentrations than the USGSCORL. No bias was observed in the data for selenium concentrations greater than $20 \mu \mathrm{g} / \mathrm{L}$. The RPD between the two laboratories was between 0.049 and 53 (table 6). A reference sample from the National Institute of Standards and Technology (NIST) was submitted to both laboratories to measure selenium concentration. The most probable value for the NIST sample was $19.97 \pm 0.16 \mu \mathrm{g} / \mathrm{L}$ (19.13-20.13 $\mu \mathrm{g} / \mathrm{L})$; neither lab result was in this range. The NWQL reported a concentration of $17.3 \mu \mathrm{g} / \mathrm{L}$, and the USGSCORL reported $21.0 \mu \mathrm{g} / \mathrm{L}$ (table 7 ). The RPD for the NIST sample compared to both the NWQL result and the USGSCORL result was 3.6 and 1.3 , respectively (table 7). Overall, results from both laboratories were acceptable, and results from both laboratories are presented in the results section of the report as selenium (NWQL), selenate (USGSCORL), and selenite (USGSCORL).

\section{Hydrogeologic Mapping}

The shallow groundwater system was mapped to improve understanding of groundwater occurrence and flow. Geographic information system (GIS) datasets produced by the mapping effort (Arnold, 2017) are available at https://doi.org/10.5066/ F70863S6. Surficial deposits and weathered Mancos Shale were mapped as a single unit, referred to as the "shallow groundwater system." The hydrogeologic mapping characterized the extent and thickness of the shallow groundwater system, altitude and configuration of the consolidated bedrock surface, and altitude and configuration of the potentiometric surface in the shallow groundwater system.

Hydrogeology of the shallow groundwater system was mapped by using lithologic and water-level data compiled from the Colorado Division of Water Resources (2012a, b), USGS (2017), Reclamation (1982), and wells drilled as part of this study (Thomas, 2015; Thomas and Arnold, 2015; 
Table 6. Selenium, selenate, and selenite concentration from the National Water Quality Laboratory (NWOL) and the U.S. Geological Survey Colorado Water Science Center Water-Quality Research Lab (USGSCORL) and the calculated relative percent difference for NWOL and USGSCORL selenium results.

$[<$, less than; --, not determined; relative percent difference, the absolute difference between replicate analyses divided by the average of the analyses and expressed as percent]

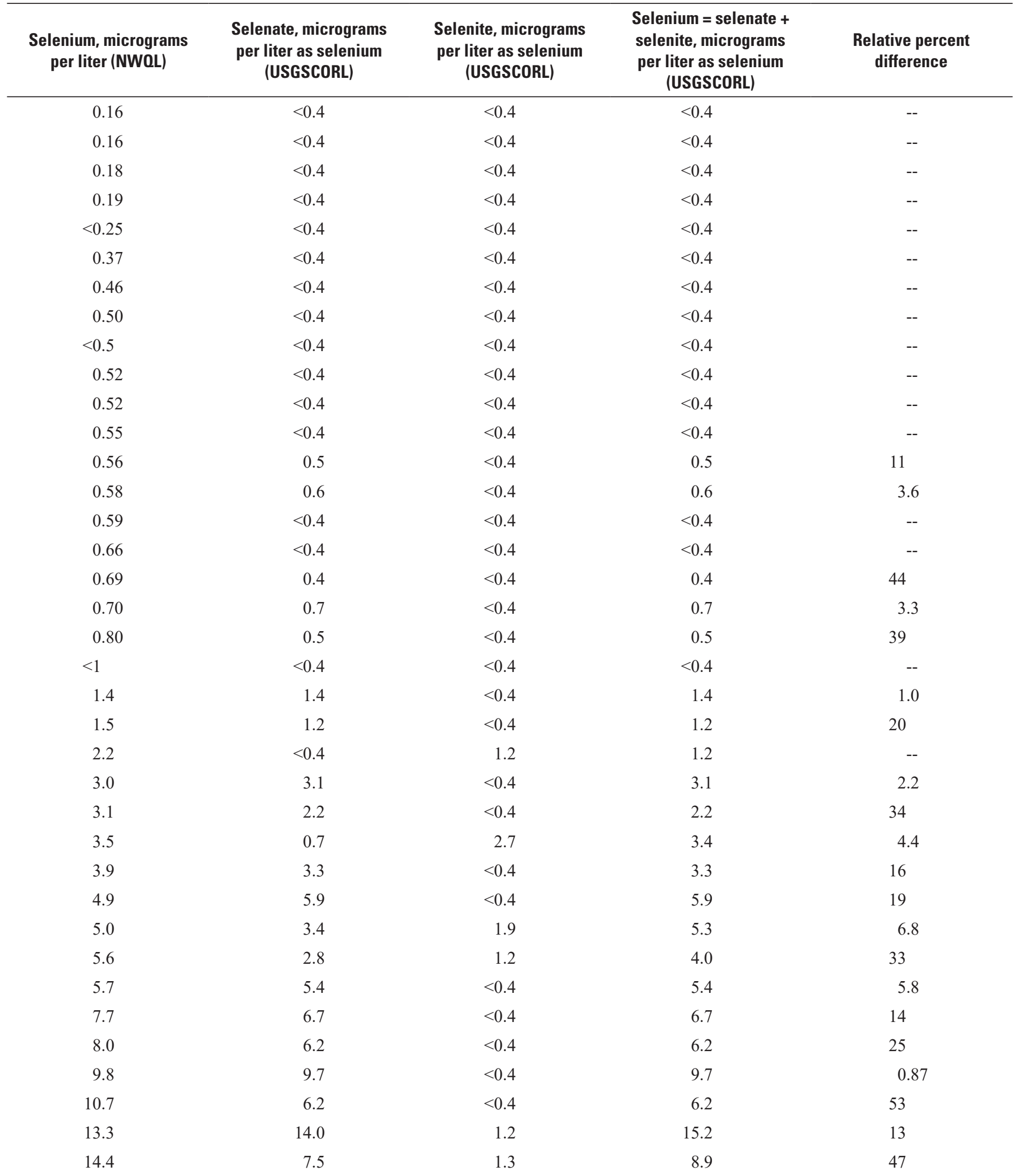


Table 6. Selenium, selenate, and selenite concentration from the National Water Quality Laboratory (NWOL) and the U.S. Geological Survey Colorado Water Science Center Water-Quality Research Lab (USGSCORL) and the calculated relative percent difference for NWOL and USGSCORL selenium results.-Continued

$[<$, less than; --, not determined; relative percent difference, the absolute difference between replicate analyses divided by the average of the analyses and expressed as percent]

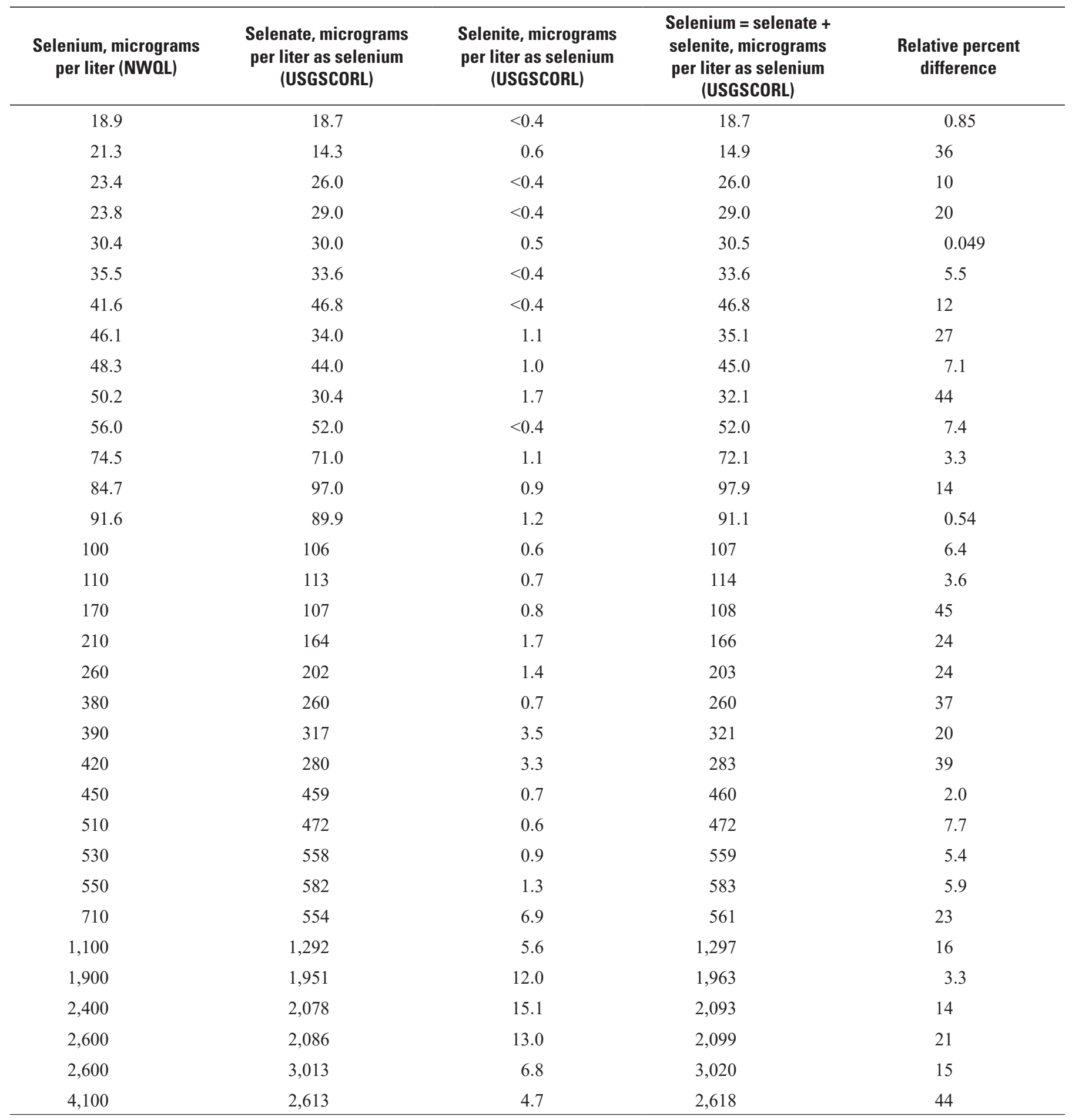


Table 7. Relative percent difference for the National Water Quality Laboratory (NWOL) and the U.S. Geological Survey Colorado Water Science Center Water-Quality Research Lab (USGSCORL) analysis results and National Institute of Standards and Technology (NIST) selenium standard.

$[\mu \mathrm{g} / \mathrm{L}$, micrograms per liter; relative percent difference, the absolute difference between replicate analyses divided by the average of the analyses and expressed as percent. Most probable value is $19.97 \mu \mathrm{g} / \mathrm{L}$. Standard was put into blank water and shipped to the NWQL and the USGSCORL]

\begin{tabular}{lclcc}
\hline Laboratory & $\begin{array}{c}\text { Selenium } \\
(\boldsymbol{\mu} \mathrm{g} / \mathrm{L})\end{array}$ & Laboratory & $\begin{array}{c}\text { Selenium } \\
(\boldsymbol{\mu g} / \mathrm{L})\end{array}$ & $\begin{array}{c}\text { Relative } \\
\text { percent } \\
\text { difference }\end{array}$ \\
\hline NIST & 19.97 & NWQL & 17.3 & 3.6 \\
NIST & 19.97 & USGSCORL & 21.0 & 1.3 \\
USGSCORL & 21.0 & NWQL & 17.3 & 4.8 \\
\hline
\end{tabular}

Arnold, 2017). The altitude of the consolidated bedrock surface underlying surficial deposits was computed as the difference between the USGS National Elevation Dataset (NED) (USGS, 2013) and the mapped thickness of surficial deposits by using GIS software (Esri, 1999-2015). Depth to water in $\mathrm{ft}$ below land surface (as represented by the potentiometric surface) was interpolated from individual depth-to-water values by kriging using GIS. Kriging was used with the mean value of coincidental points to generate a smooth geostatistical fit to varied and sometimes inconsistent values at a local scale. Because depth to water was measured for various dates, depth intervals, and hydrologic conditions, water level should be considered representative of general conditions rather than precise conditions for a specific location or time. Altitude of the potentiometric surface in feet above North American Vertical Datum of 1988 (NAVD 88) for the shallow groundwater system was computed as the difference between the NED and depth-to-water potentiometric surface. Saturated thickness of the shallow groundwater system was computed as the difference between the altitude of the potentiometric surface and the altitude of consolidated bedrock.

\section{Water-Level Measurements}

Data on water level, measured as depth to water below land surface, were collected throughout the period of study, from 2012 to 2016. Water level was measured with an electric water-level tape at the time of drilling, during each of the water-quality sampling events, and during monthly visits starting in January 2015 by using methods outlined in Cunningham and Schalk (2011). Monthly manual water levels were measured in all 30 wells from January 2015 to December 2016 by using an electric water-level tape. The water-level altitude was determined by subtracting the measured depth to water in feet below land surface from the land-surface altitude above NAVD 88 at each well location (table 1). Continuous water-level data were collected at 10 wells (table 1) from April 2015 to December 2016 by using submersible pressure transducers with vented cables. Both monthly data and continuous data are stored in the NWIS at https://doi.org/10.5066/F7P55KJN.

The pressure transducers were installed by using standard methods for equipment installation, as outlined in Cunningham and Schalk (2011). The pressure transducers were suspended in the well on the vented communication cable, allowing data to be downloaded without disturbing the instrument. The initial water level for each transducer was set to the value measured prior to deploying the transducer. The transducer was programmed to record the water level every 15 minutes. Monthly manual water-level measurements were used to correct the continuous data for instrument drift following USGS guidelines (Freeman and others, 2004).

\section{Single-Hole Aquifer Test and Analysis}

Single-hole aquifer tests or slug tests were used to characterize aquifer properties and involved rapidly changing the water level in a well and measuring the rate of water-level response (Cunningham and Schalk, 2011). Appropriate analytical methods were applied to the data collected to estimate hydraulic conductivity.

Standardized techniques were used to conduct an instantaneous change in head (slug) test with a mechanical slug and submersible pressure transducer (Cunningham and Schalk, 2011). An electric water-level tape was used to measure static water level in a well prior to each test. Water level was measured to the nearest hundredth of a foot below the established measuring point for each well. The pressure transducer was placed below the depth at which the slug would be deployed. The water level was then measured to determine if it was close to the initial static value. The slug was lowered quickly into the well to a depth where the top of the slug was $1 \mathrm{ft}$ below the water surface, which resulted in a rise in the water level (slugin test). The water level was recorded with the pressure transducer and was allowed to recover to at or near its initial value. The slug was then quickly removed from the well, causing a drop in the water level (slug-out test). Again, the water level was recorded with the pressure transducer and was allowed to recover to at or near its initial static value. This process was completed at least once, twice when time permitted, to provide up to four tests per well.

The methods of Bouwer and Rice (1976), Butler and others (2003), or Cooper-Greene (Greene and Shapiro, 1998) were used to analyze slug-test data according to implementation noted in the spreadsheets developed by Halford and Kuniansky (2002). The slug-test data were then copied into the spreadsheets, along with information about the slug method, slug dimensions, well dimensions, and the aquifer (fig. 4). The spreadsheet was used to generate plots showing changes in water level during each slug test (fig. 5). Then, the appropriate worksheet for the chosen analysis method was used to calculate the hydraulic conductivity (fig. 6). The 
Well 1 Construction

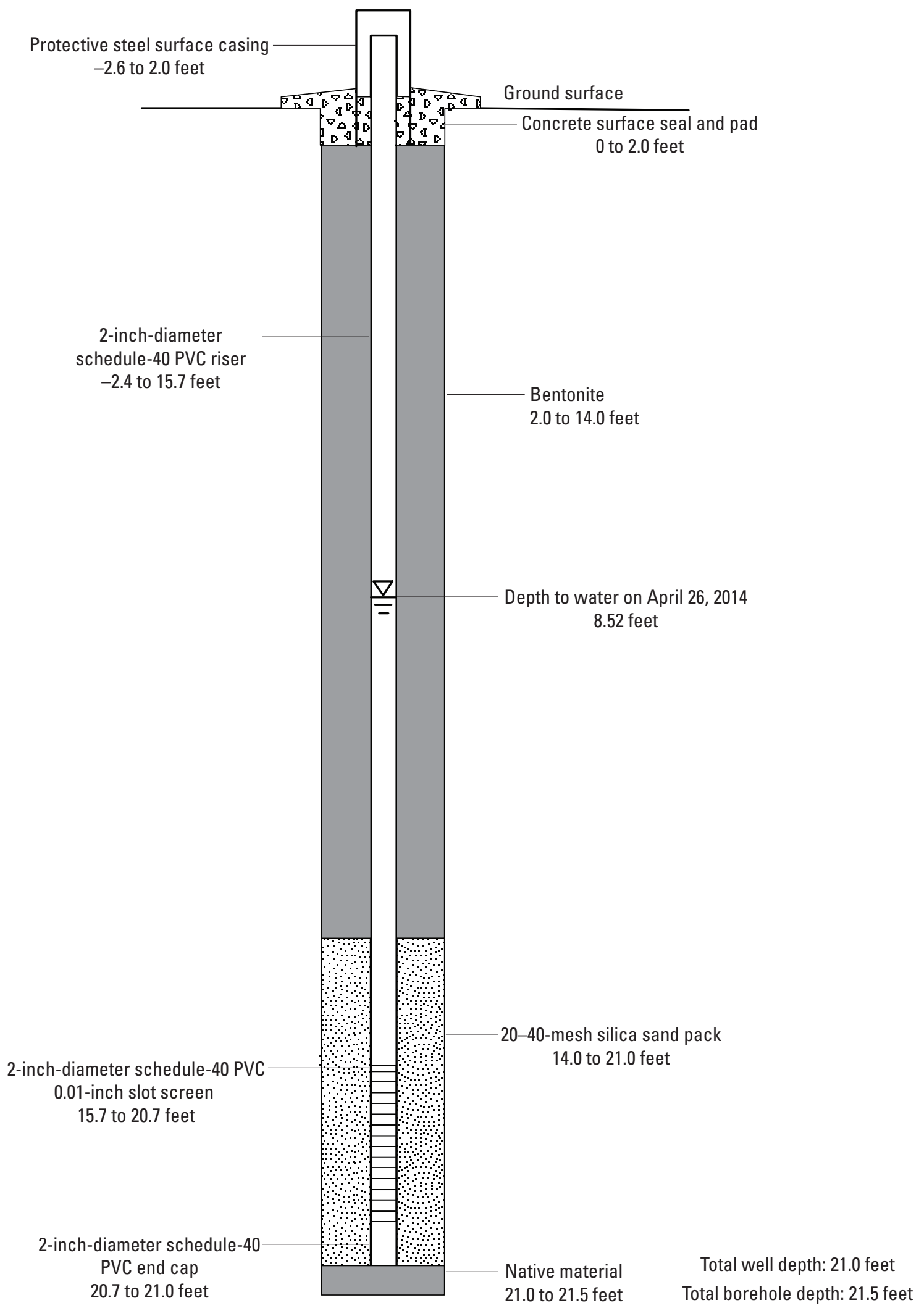

Figure 4. Example of well diagram for well 1. (PVC, polyvinyl chloride; depth expressed in feet below land surface, negative values indicate feet above land surface) 


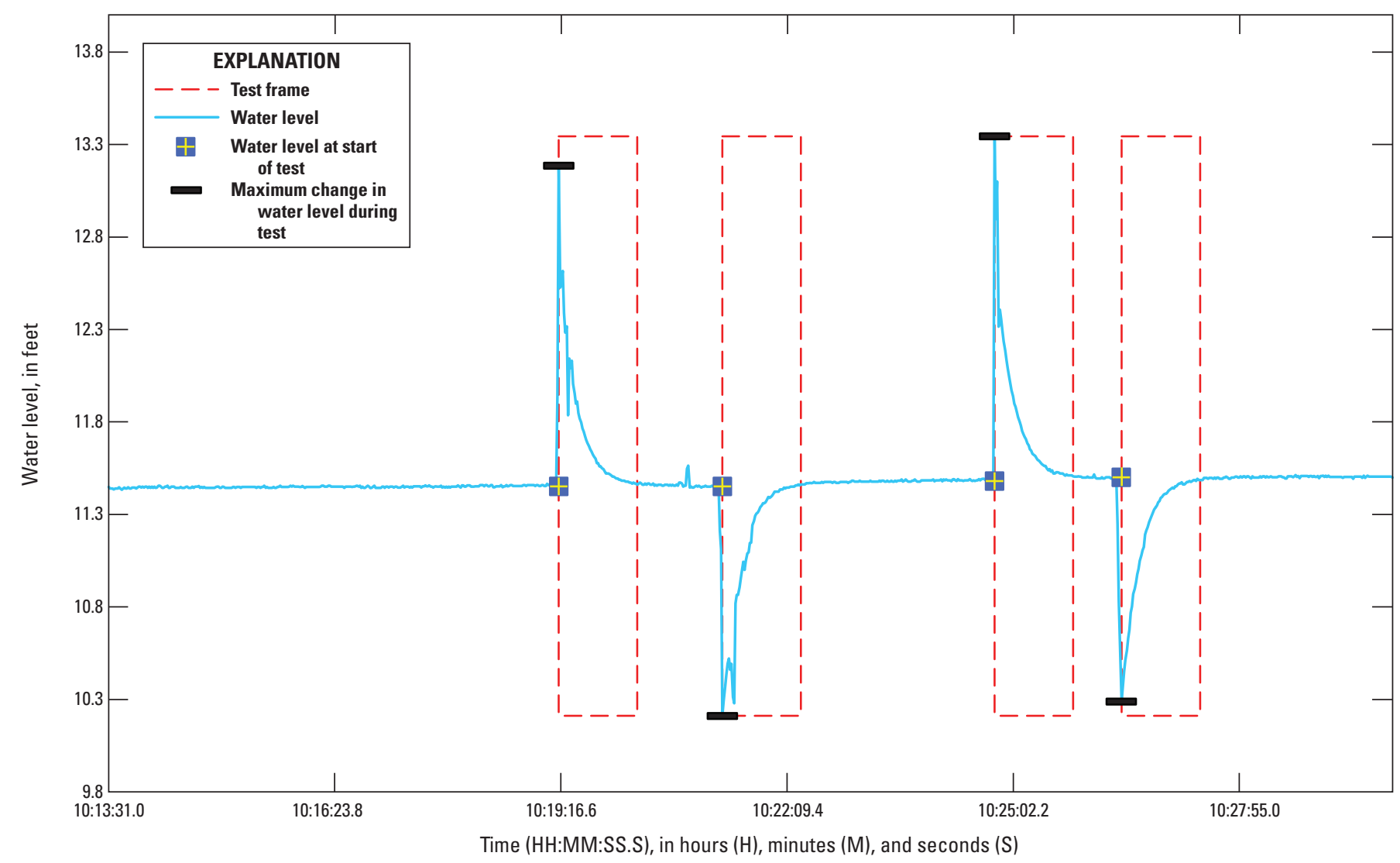

TEST DURATION: $\quad 60 \mathrm{sec} \quad \Gamma$ Focus on an Event

\begin{tabular}{ccrrrr} 
TEST & $\mathbf{t}_{\mathbf{0}}$ & $\mathbf{W L}_{\text {REF }}$ & $\mathbf{W L}_{\text {MAX }}$ & $\mathbf{y}_{\mathbf{0}}$ & \multicolumn{1}{c}{ ANALYZE } \\
\hline 1 & $10: 19: 15.0$ & 11.450 & 13.186 & 1.74 & TRUE \\
2 & $10: 21: 20.0$ & 11.450 & 10.211 & 1.24 & TRUE \\
3 & $10: 24: 48.0$ & 11.480 & 13.344 & 1.86 & TRUE \\
4 & $10: 26: 25.0$ & 11.500 & 10.289 & 1.21 & TRUE \\
\hline
\end{tabular}

Figure 5. Example of slug-test trace for well 1 from spreadsheets developed by Halford and Kuniansky, 2002. (sec, second; $\mathrm{t}_{0^{\prime}}$, start time of test; $\mathrm{WL}_{\mathrm{REF}}$, water level at start of test, in feet; $\mathrm{WL}_{\mathrm{MAx}^{\prime}}$ maximum change in water level during test, in feet; $\mathrm{y}_{0^{\prime}}$ displacement in feet for test; ANALYZE is TRUE for tests used to estimate hydraulic conductivity.)

resulting plots show the data from each test and the predictive curve based on the selected analytical method. Choice of method is based on the best visual fit of the predictive curve to the actual data. Examples of a well diagram, a slug-test trace, and a slug-test analysis are provided in figures 4,5 , and 6, respectively. Review and approval of slug-test results followed USGS groundwater-quality-assurance procedures (Putnam and Hansen, 2014).

\section{Water-Table Fluctuation Method}

A graphical approach to the water table fluctuation (WTF) method was used to estimate the amount of recharge to the unconfined portion of the shallow groundwater system. The antecedent recession curves were extrapolated manually to obtain the peak water-table rise $\left(\Delta \mathrm{H}\left(\mathrm{t}_{\mathrm{j}}\right)\right)$ on the basis of visual inspection of the entire dataset (Delin and others, 2007). Estimates of $\Delta \mathrm{H}\left(\mathrm{t}_{\mathrm{j}}\right)$ are identified by using the difference between the peak of a water-level rise and the value of the extrapolated antecedent recession curve at the time of the peak. The recession curve is a trace of how the well hydrograph would have looked had there not been any change in the water table (appendix 1). Specific yield (Sy) values were estimated from knowledge of the lithologic material for each well and compared to values derived by Johnson (1967). 


\section{Well 1}

INPUT

Construction:

Casing diameter $=2.046$ in.

Annulus diameter $=\quad 8$ in.

Depths to:

Water level =

Top of screen $=$

Base of screen $=$

Top of aquifer $=$

Base of aquifer $=$

Annular fill:

across screen: fine sand

above screen: bentonite
Date: $11 / 18 / 2014$

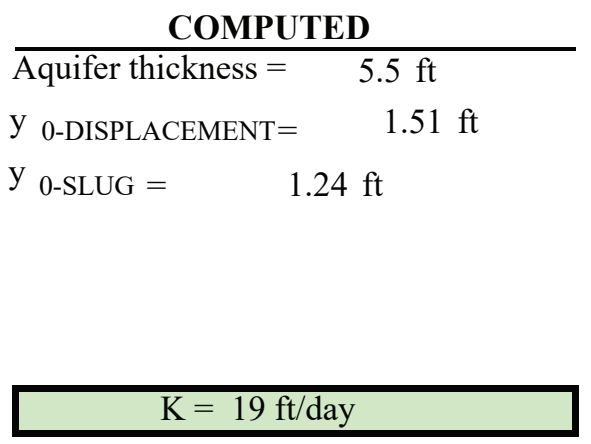

Bouwer and Rice

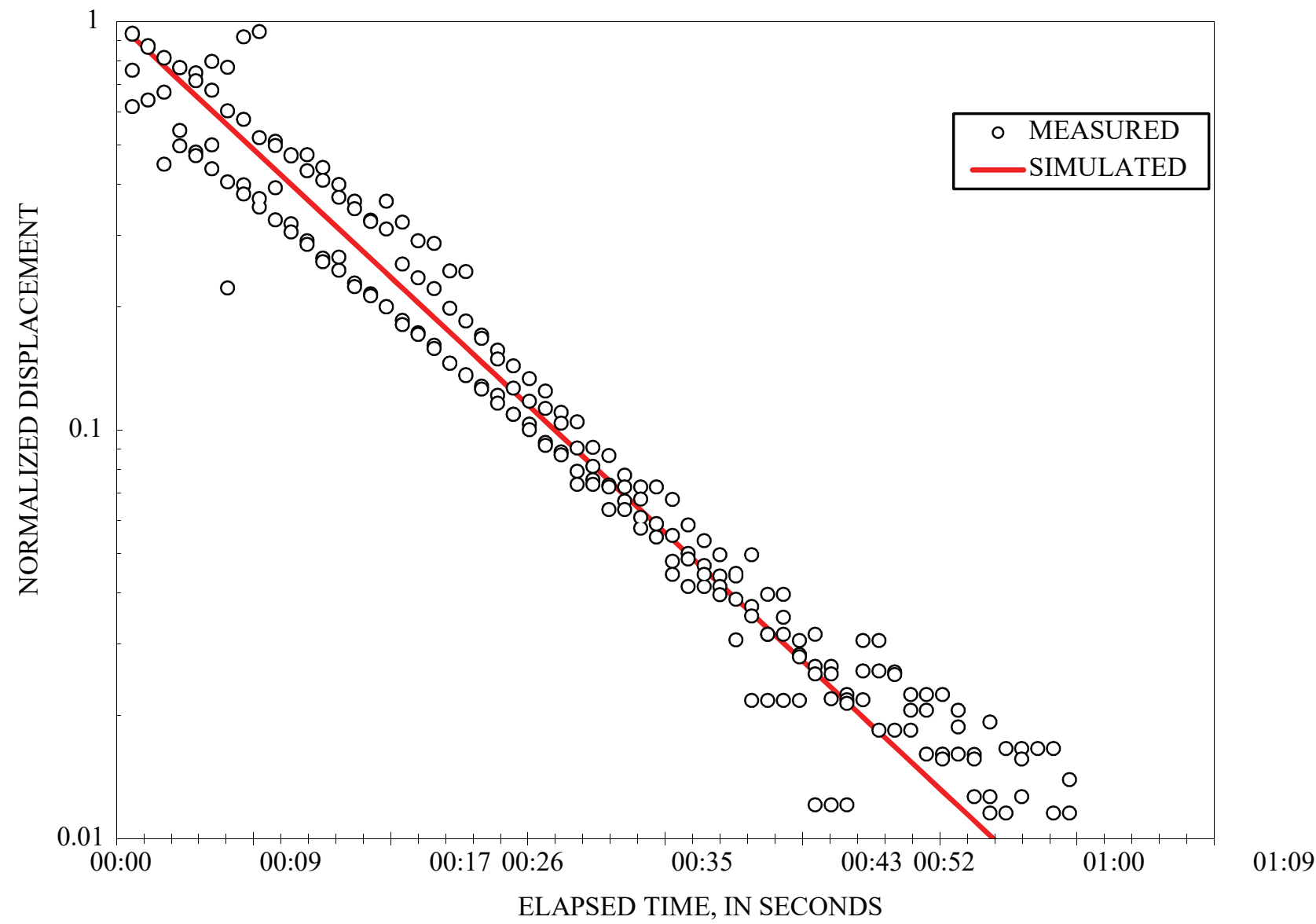

Figure 6. Example of slug-test analysis at well 1 from spreadsheets developed by Halford and Kuniansky, 2002. (in., inch; $\mathrm{ft}$, foot; $\mathrm{y}_{\text {0-displacement' }}$ theoretical displacement; $\mathrm{y}_{0 \text {-slug' }}$ actual displacement; $\mathrm{K}$, hydraulic conductivity; $\mathrm{ft} / \mathrm{d}$, foot per day; depth expressed in feet below land surface. Method selected refers to Bouwer and Rice, 1976) 


\section{Groundwater-Age Calculations}

Mean groundwater ages were computed from concentrations of tritium, tritiogenic helium-3 $\left({ }^{3} \mathrm{He}_{\text {trit }}\right)$, and $\mathrm{SF}_{6}$ by using the lumped-parameter modeling software TracerLPM (Jurgens and others, 2012). (Helium-3 is produced from the radioactive decay of tritium.) Concentrations of ${ }^{3} \mathrm{He}_{\text {trit }}$ were calculated from measured helium-4 concentrations and helium-3/ helium-4 ratios by using methods described by Solomon and Cook (2000). The calculations assumed a helium-3/helium-4 ratio of $2 \times 10^{-8}$ for any terrigenic helium in the samples (Mamyrin and Tolstikhin, 1984). For $\mathrm{SF}_{6}$, TracerLPM computations are based on the $\mathrm{SF}_{6}$ concentration in air that would be in equilibrium with the measured concentration in groundwater. The equilibrium $\mathrm{SF}_{6}$ concentration in air was calculated by using methods described by Busenberg and Plummer (2000) and Jurgens and others (2016). The ${ }^{3} \mathrm{He}_{\text {trit }}$ and $\mathrm{SF}_{6}$ calculations also require estimates of groundwater recharge temperature and concentrations of excess air in the samples, which were calculated from the noble gas and $\mathrm{N}_{2}$ data by using methods described by Aeschbach-Hertig and others (1999). A leastsquares fitting routine was used to calculate mean groundwater age, recharge temperature, and excess air and to minimize the sum of the weighted squared difference (chi-squared, $\chi^{2}$ ) between measured and modeled age-tracer concentrations (for mean-age calculations) and between measured and modeled gas concentrations (for recharge-temperature and excess-air calculations) (Aeschbach-Hertig and others, 1999; Jurgens and others, 2012).

\section{Loading Calculations}

Instantaneous selenium load in surface water was calculated by using an instantaneous streamflow, in cubic feet per second, and associated selenium concentration, in micrograms per liter, and was converted to pounds per day by a unit conversion factor (0.005395). Mean instantaneous selenium load, in pounds per day, was determined for each month by taking the mean of the instantaneous selenium loads. Monthly mean selenium load, in pounds per month, was determined by multiplying the mean instantaneous selenium load for that month by the number of days in the month.

\section{Groundwater Quality}

Monitoring wells were sampled on multiple occasions to understand groundwater quality, sources of recharge, and groundwater age in the shallow groundwater system. The 30 -well network provided a spatial distribution to understand groundwater quality over the study area. Wells were completed in both surficial deposits and the weathered Mancos Shale to evaluate the role that geologic material would have with respect to groundwater quality. Samples were collected during both the irrigation and nonirrigation seasons to evaluate seasonality. Although data on many properties and constituents were collected as part of this study (table 3), only select properties and constituents are discussed here (tables 8 and 9). The following major ions and nutrients are not discussed: bromide, silica, ammonia, orthophosphate, and total nitrogen. The following trace elements are discussed: iron, lithium, manganese, strontium, boron, selenate, selenite, selenium, and uranium (table 9). All data collected as part of this study are available from the NWIS at https://doi.org/10.5066/F7P55KJN.

\section{General Water-Quality Indicators}

Field properties collected as part of the study include dissolved oxygen, $\mathrm{pH}$, specific conductance, water temperature, turbidity, and alkalinity (table 8). Dissolved oxygen, water temperature, and turbidity were relatively similar among all wells both spatially and temporally. $\mathrm{pH}$ is the only property that exhibited a significant $(p=0.007)$ though small difference between the irrigation and nonirrigation seasons. No spatial pattern or relation between $\mathrm{pH}$ and geologic material was observed.

Specific conductance is proportional to the concentration of major dissolved constituents (cations and anions) (Hem, 1985) and has been used as a surrogate for dissolved solids and salinity. No temporal pattern in specific conductance values of groundwater was observed between the irrigation and nonirrigation seasons; however, the specific conductance of applied irrigation water was considerably less than that of groundwater. For comparison to groundwater, specific conductance data from the Uncompahgre River at Colona, Colo., (fig. 1) was used because it is located near the beginning of the irrigation project and represents the initial input into the irrigation system. Specific conductance values measured in groundwater range from 1,530 to 43,600 microsiemens per centimeter at 25 degrees Celsius $\left(\mu \mathrm{S} / \mathrm{cm}\right.$ at $\left.25^{\circ} \mathrm{C}\right)$, with a median value of $4,160 \mu \mathrm{S} / \mathrm{cm}$ at $25^{\circ} \mathrm{C}$ (table 8). In comparison, specific conductance of surface water at the Uncompahgre River at Colona (fig. 1) was $500 \mu \mathrm{S} / \mathrm{cm}$ at $25^{\circ} \mathrm{C}$ (annual statistic for water year 2011). Data were obtained from the NWIS at https://doi.org/10.5066/F7P55KJN. Because irrigation water was thought to be the primary source of recharge to the groundwater system, it would seem possible that specific conductance of groundwater would decrease during the irrigation season; however, no decrease was observed. One interpretation of this observation is that the monitoring wells were relatively far from the sources of recharge and that water from those wells had been in contact with aquifer materials for a long period of time relative to newly recharged water. In addition to water residence times near aquifer materials, the existence and distribution of soluble salts in the aquifer itself are an important source of dissolved solids throughout the study area (Mast and others, 2014; Mills and others, 2016). High specific conductance values indicate that groundwater is an important source of salinity to surface water.

Major-ion data were used to identify the source of the high concentrations of dissolved solids, with specific conductance again used as a surrogate for dissolved solids. Groundwater types in the study area were calcium sulfate, 
Table 8. Summary statistics for select field properties, major ions, and nutrients for water collected from the monitoring wells, east side of the Uncompahgre River, lower Gunnison River Basin, Colorado, 2013-16.

[mg/L, milligram per liter; <, less than; $\mu \mathrm{S} / \mathrm{cm}$ at $25^{\circ} \mathrm{C}$, microsiemen per centimeter at 25 degrees Celsius; $\mathrm{NC}$, not calculated; NA, not applicable]

\begin{tabular}{|c|c|c|c|c|c|}
\hline $\begin{array}{c}\text { Constituent } \\
\text { (units) }\end{array}$ & $\begin{array}{c}\text { Number } \\
\text { of samples }\end{array}$ & $\begin{array}{l}\text { Number } \\
\text { of censored } \\
\text { values }\end{array}$ & $\begin{array}{l}\text { Minimum } \\
\text { value }\end{array}$ & $\begin{array}{l}\text { Maximum } \\
\text { value }\end{array}$ & $\begin{array}{c}\text { Median } \\
\text { value }\end{array}$ \\
\hline Dissolved oxygen $(\mathrm{mg} / \mathrm{L})$ & 70 & 61 & $<0.5$ & 6.9 & $\mathrm{NC}$ \\
\hline $\mathrm{pH}$ (standard units) & 70 & 0 & 6.6 & 7.3 & 6.8 \\
\hline Specific conductance $\left(\mu \mathrm{S} / \mathrm{cm}\right.$ at $\left.25^{\circ} \mathrm{C}\right)$ & 70 & 0 & 1,530 & 43,600 & 4,160 \\
\hline Water temperature (degrees Celsius) & 70 & 0 & 8 & 17 & 13 \\
\hline Alkalinity $(\mathrm{mg} / \mathrm{L})$ as calcium carbonate & 70 & 0 & 182 & 1,630 & 414 \\
\hline Turbidity (nephelometric turbidity ratio units) & 59 & NA & 0.1 & 130 & 1 \\
\hline Dissolved solids (mg/L) & 70 & 0 & 926 & 53,000 & 4,000 \\
\hline Calcium(mg/L) & 70 & 0 & 168 & 617 & 499 \\
\hline Magnesium (mg/L) & 70 & 0 & 42 & 2,500 & 250 \\
\hline Potassium (mg/L) & 70 & 0 & 2 & 53 & 9 \\
\hline Sodium (mg/L) & 70 & 0 & 62 & 13,300 & 312 \\
\hline Bicarbonate $(\mathrm{mg} / \mathrm{L})$ & 70 & 0 & 222 & 1,980 & 518 \\
\hline Chloride (mg/L) & 70 & 0 & 0.910 & 3,880 & 28.2 \\
\hline Sulfate $(\mathrm{mg} / \mathrm{L})$ & 70 & 0 & 527 & 34,400 & 2,210 \\
\hline Nitrite plus nitrate ( $\mathrm{mg} / \mathrm{L}$ as nitrogen) & 70 & 19 & $<0.04$ & 550 & 4.8 \\
\hline Nitrite $(\mathrm{mg} / \mathrm{L}$ as nitrogen) & 70 & 19 & $<0.001$ & 7.1 & 0.20 \\
\hline Carbon, organic, dissolved, water, filtered $(\mathrm{mg} / \mathrm{L})$ & 60 & 0 & 1.82 & 82 & 6.8 \\
\hline Carbon dioxide $(\mathrm{mg} / \mathrm{L})$ & 27 & NA & 14.2 & 423 & 62.3 \\
\hline
\end{tabular}

mixed-cation sulfate, and sodium sulfate (fig. 7). Through use of specific conductance values, major-ion data were grouped into 25th, 50th, and 75th percentiles (fig. 7). Specific conductance values in the 75th percentile and greater were found in association with sodium-sulfate-type waters, and specific conductance values in the 25th percentile were found in association with calcium-sulfate-type waters (fig. 7). This finding is similar to the findings of Mills and others (2016), who found the dissolution of sodium sulfate salts to be an important mechanism in groundwater geochemistry.

\section{Nitrate and Dissolved Organic Carbon}

Wells in the study area had high concentrations of nitrate and dissolved organic carbon (table 8). Concentrations of dissolved nitrite in groundwater ranged from below the limit of detection $(<0.001)$ to 7.1 milligrams per liter as nitrogen, with a median concentration of 0.20 milligrams per liter as nitrogen (table 8), indicating that the measurement of nitrite plus nitrate consisted mostly of nitrate and is referred to as nitrate. Concentrations of dissolved nitrite plus nitrate (referred to as nitrate) in groundwater ranged from below the limit of detection $(<0.04)$ to 550 milligrams per liter as nitrogen, with a median concentration of 4.8 milligrams per liter as nitrogen (table 8). Colorado agricultural standard for nitrate in groundwater is 100 milligrams per liter as nitrogen (CDPHE, 2016). Dissolved organic carbon concentrations in groundwater ranged from 1.82 to $82 \mathrm{mg} / \mathrm{L}$, with a median concentration of $6.8 \mathrm{mg} / \mathrm{L}$ (table 8 ). A high degree of variability was observed in measurements of both nitrate and dissolved organic carbon concentrations.

The primary source of nitrate and dissolved organic carbon in groundwater is the Mancos Shale. Nitrogen and carbon are found primarily in organic forms in the Mancos Shale (Holloway and Smith, 2005). As the shale weathers, groundwater becomes enriched with nitrate and dissolved organic carbon (Morrison and others, 2012; Mast and others, 2014; Linard and others, 2016). Although it is possible that agriculture was a source of some of the nitrate in groundwater, the primary source is the Mancos Shale. Concentrations of nitrate and dissolved organic carbon from samples collected during this study were highly correlated $(r=0.86, p<0.01)$. Nitrate concentrations measured in this study are comparable to concentrations measured in groundwater and pore water in areas of the Mancos Shale unaffected by irrigated agriculture (Morrison and others, 2012; Mast and others, 2014; Linard and others, 2016), indicating that the Mancos Shale is the primary source of both of these constituents in the shallow groundwater system. 


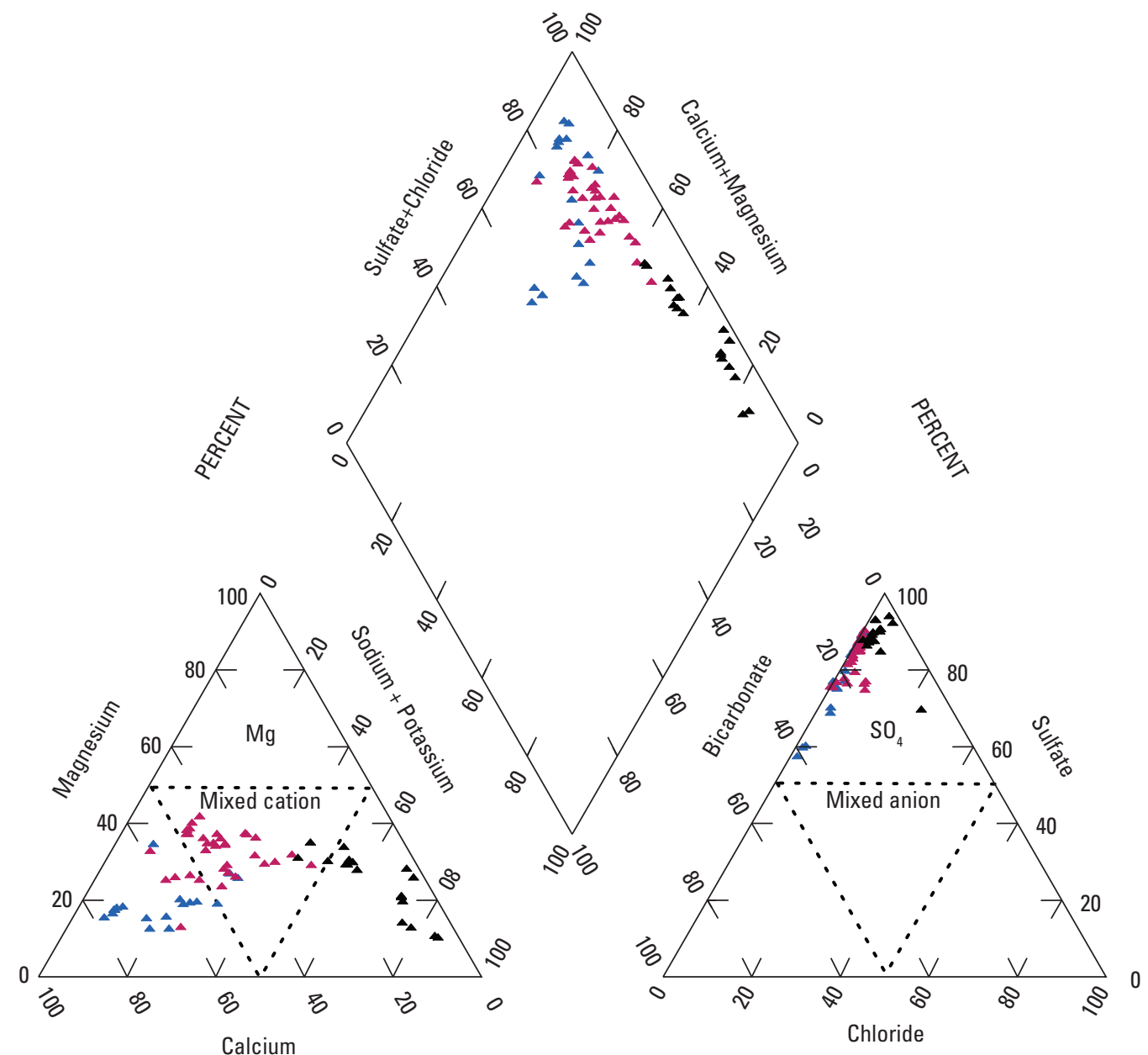

PERCENT

EXPLANATION

Specific conductance

of groundwater

- High - 75th percentile

- Middle-50th percentile

- Low-25th percentile

Figure 7. Water types grouped by the 25th, 50th, and 75th percentiles of specific conductance of groundwater, 2013-16.

\section{Selenium}

Prior to this study, little was known about the concentrations of selenium in the shallow groundwater system, even though groundwater was believed to be an appreciable source of selenium to surface water (Morrison and others, 2012; Mast and others, 2014; Linard and others, 2016). Concentrations of dissolved selenium ranged from below the limits of detection to $4,100 \mu \mathrm{g} / \mathrm{L}$, with a median concentration of $14 \mu \mathrm{g} / \mathrm{L}$ (table 9). Only three values were less than detection. A total of 70 selenium concentration samples were collected during the study: 27 samples (39 percent) were below the chronic aquatic-life standard of $4.6 \mu \mathrm{g} / \mathrm{L}, 37$ samples (53 percent) were below the acute aquatic-life standard of $18.4 \mu \mathrm{g} / \mathrm{L}$ (CDPHE, 2016), and 33 samples (47 percent) exceeded both standards. A total of 23 samples (33 percent) exceeded the primary drinking water standard of $50 \mu \mathrm{g} / \mathrm{L}$. Selenium was present mostly in its oxidized form, selenate (table 9). Mass ratios of selenite to total selenium ranged from 0.0013 to 0.79 , with a median of 0.058 (table 9), indicating that typically less than 6 percent of the total selenium in groundwater samples was selenite.

No spatial (fig. 8) or temporal (fig. 9) pattern emerged with regard to selenium concentration. Selenium concentrations were not significantly different between the irrigation and nonirrigation seasons $(p=0.202)$ (fig. 9), despite the influx of irrigation water, which generally has selenium concentrations much less than that observed in groundwater. Selenium concentrations had a high degree of spatial variability across the 30-well network (fig. 8). Selenium concentrations in surficial deposits (median of $23 \mu \mathrm{g} / \mathrm{L}$ ) were not significantly greater than selenium concentrations in the weathered Mancos Shale (median of $5 \mu \mathrm{g} / \mathrm{L}, \mathrm{p}=0.0755$ ) (fig. 9). 
Table 9. Summary statistics for select trace elements for water collected from the monitoring wells, east side of the Uncompahgre River, lower Gunnison River Basin, Colorado, 2013-16.

$[\mu \mathrm{g} / \mathrm{L}$, microgram per liter; <, less than; NC, not calculated; NA, not applicable]

\begin{tabular}{|c|c|c|c|c|c|}
\hline Constituent & $\begin{array}{c}\text { Number } \\
\text { of samples }\end{array}$ & $\begin{array}{c}\text { Number } \\
\text { of censored } \\
\text { values }\end{array}$ & $\begin{array}{c}\text { Minimum } \\
\text { value }\end{array}$ & $\begin{array}{l}\text { Maximum } \\
\text { value }\end{array}$ & $\begin{array}{c}\text { Median } \\
\text { value }\end{array}$ \\
\hline Iron $(\mu \mathrm{g} / \mathrm{L})$ & 70 & 30 & $<4,<8,<12,<16,<20,<24,<28,<40,<80,<400$ & 8,650 & $\mathrm{NC}$ \\
\hline Lithium $(\mu \mathrm{g} / \mathrm{L})$ & 70 & 0 & 74.9 & 4,860 & 257 \\
\hline Manganese $(\mu \mathrm{g} / \mathrm{L})$ & 70 & 2 & $<0.8,<4.0$ & 1,430 & 429 \\
\hline Selenate ( $\mu \mathrm{g} / \mathrm{L}$ as selenium $)$ & 70 & 16 & $<0.4$ & 3,010 & $\mathrm{NC}$ \\
\hline Selenite $(\mu \mathrm{g} / \mathrm{L}$ as selenium $)$ & 70 & 37 & $<0.4$ & 15.1 & $\mathrm{NC}$ \\
\hline Selenite/total selenium mass ratio & 70 & NA & 0.001 & 0.79 & 0.058 \\
\hline Selenium $(\mu \mathrm{g} / \mathrm{L})$ & 70 & 3 & $<0.25,<0.5,<1.0$ & 4,100 & 14 \\
\hline
\end{tabular}

\section{Redox Indicators}

The redox process is the transfer of electrons from one chemical species to another (Langmuir, 1997). The chemical that donates the electron is "oxidized," and the chemical that accepts that electron is "reduced." Naturally occurring microbes mediate many of these processes and, in turn, use the energy generated to support their metabolism. Dissolved and solid organic carbon in aquifer systems are typically the source of the electrons. The electron acceptors, listed in order of most to least energetically favorable, are dissolved oxygen, nitrate, manganese, iron, sulfate, and carbon dioxide. Oxygen reduction is the first process in the redox sequence, followed by nitrate reduction (most commonly in the form of denitrification). Reduction of sulfate and carbon dioxide occur under highly anoxic conditions and are the last processes in the sequence.

Redox processes are important controls on selenium speciation and mobility. Selenate, the most mobile form of selenium, is generally mobile under oxygen- and nitratereducing conditions. However, once nitrate concentrations are reduced to low levels (less than $5 \mathrm{mg} / \mathrm{L}$ ) under nitratereducing conditions, selenate can be reduced and removed from solution (Bailey and others, 2014). A previous study found that nitrate concentrations as low as 5 milligrams per liter as nitrogen were enough to inhibit the reduction of selenate (Bailey and others, 2012). The median nitrate concentration measured in this study was 4.8 milligrams per liter as nitrogen (table 8).

Several redox processes were identified in the shallow groundwater system by using a redox framework based on measured concentrations of dissolved oxygen, nitrate, manganese, iron, and sulfate (McMahon and Chapelle, 2008). In this framework, oxic is defined as having a dissolved oxygen concentration of at least $0.5 \mathrm{mg} / \mathrm{L}$ (McMahon and Chapelle, 2008). Suboxic, anoxic, and mixed (anoxic) have dissolved oxygen concentrations of less than $0.5 \mathrm{mg} / \mathrm{L}$ and are further defined by using nitrate, manganese, iron, and sulfate concentrations (McMahon and Chapelle, 2008). The conditions in the shallow groundwater system are generally anoxic. Of the 70 samples, 58 had sufficient data to categorize redox processes by using the framework. These processes were then sorted into 3 groups according to their redox category: group 1 was oxic and mixed (oxic-anoxic), group 2 was mixed (anoxic), and group 3 was anoxic. No pattern or relationship was observed between redox category and spatial distribution, geologic material, or irrigation and nonirrigation seasons. Selenate concentration data were evaluated by redox group. Samples in group 1 had a median selenate concentration of $26 \mu \mathrm{g} / \mathrm{L}$ as selenium, samples in group 2 had a median selenate concentration of $106 \mu \mathrm{g} / \mathrm{L}$ as selenium, and samples in group 3 had a median selenate concentration less than $0.4 \mu \mathrm{g} / \mathrm{L}$ as selenium (fig. 10). Selenate concentrations in groups 1 and 2 were not significantly different from one another $(\mathrm{p}=0.061)$, whereas concentrations in group 3 were significantly lower than concentrations in groups 1 and $2(p<0.01)$. This pattern is consistent with results from previous studies (Morrison and others, 2012; Mast and others, 2014; Linard and others, 2016), showing that highly reducing conditions maintain selenate concentrations at low levels.

\section{Other Trace Elements}

In addition to selenium, several other trace elements were detected in high concentrations in groundwater, most notably boron, iron, manganese, lithium, strontium, and uranium (table 9), presumably sourced from the Mancos 


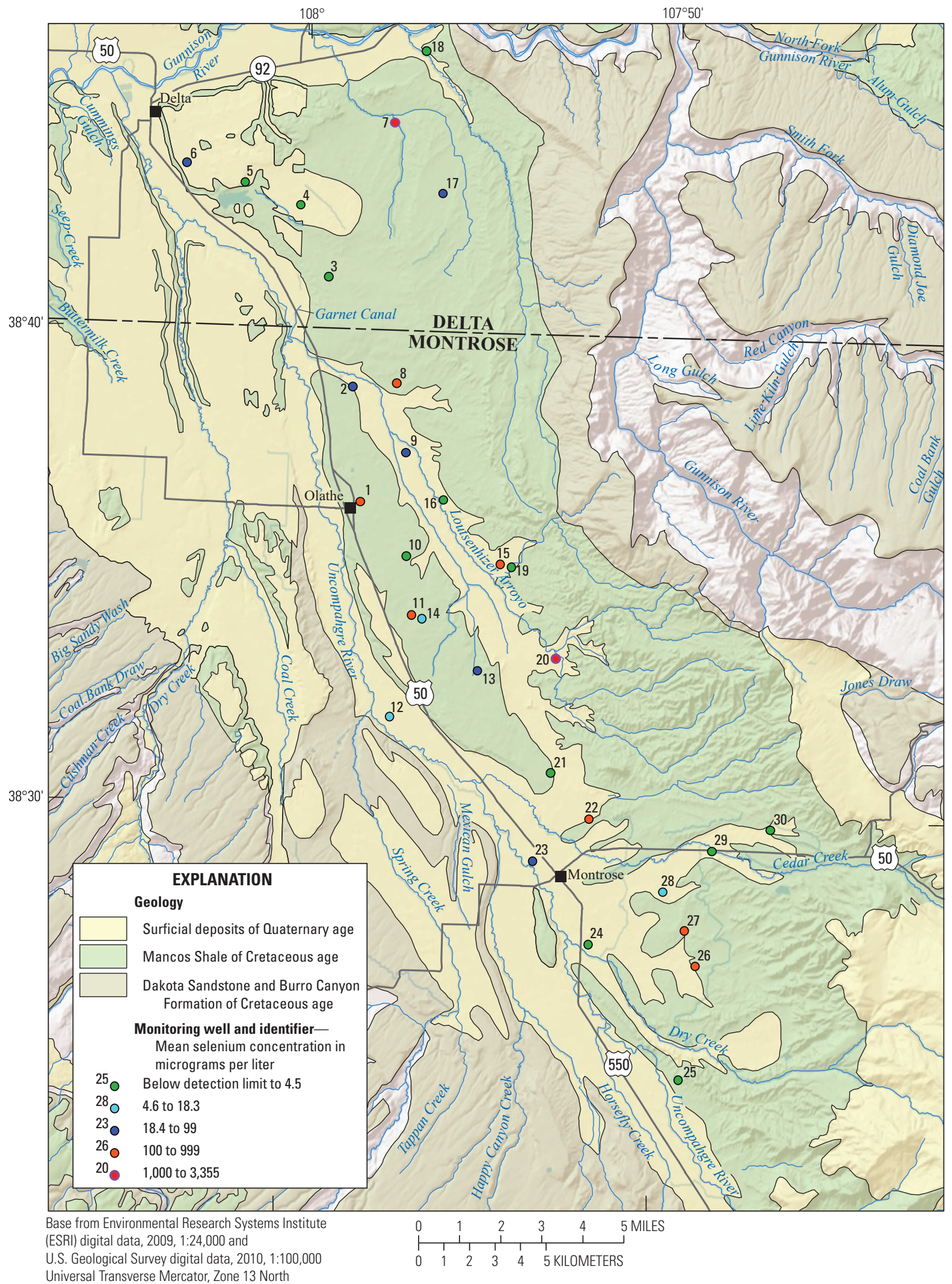

Universal Transverse Mercator, Zone 13 North

North American Datum 1983

Figure 8. Mean selenium concentration at each monitoring well in the study area, 2013-16. Geology modified from Tweto (1979). 

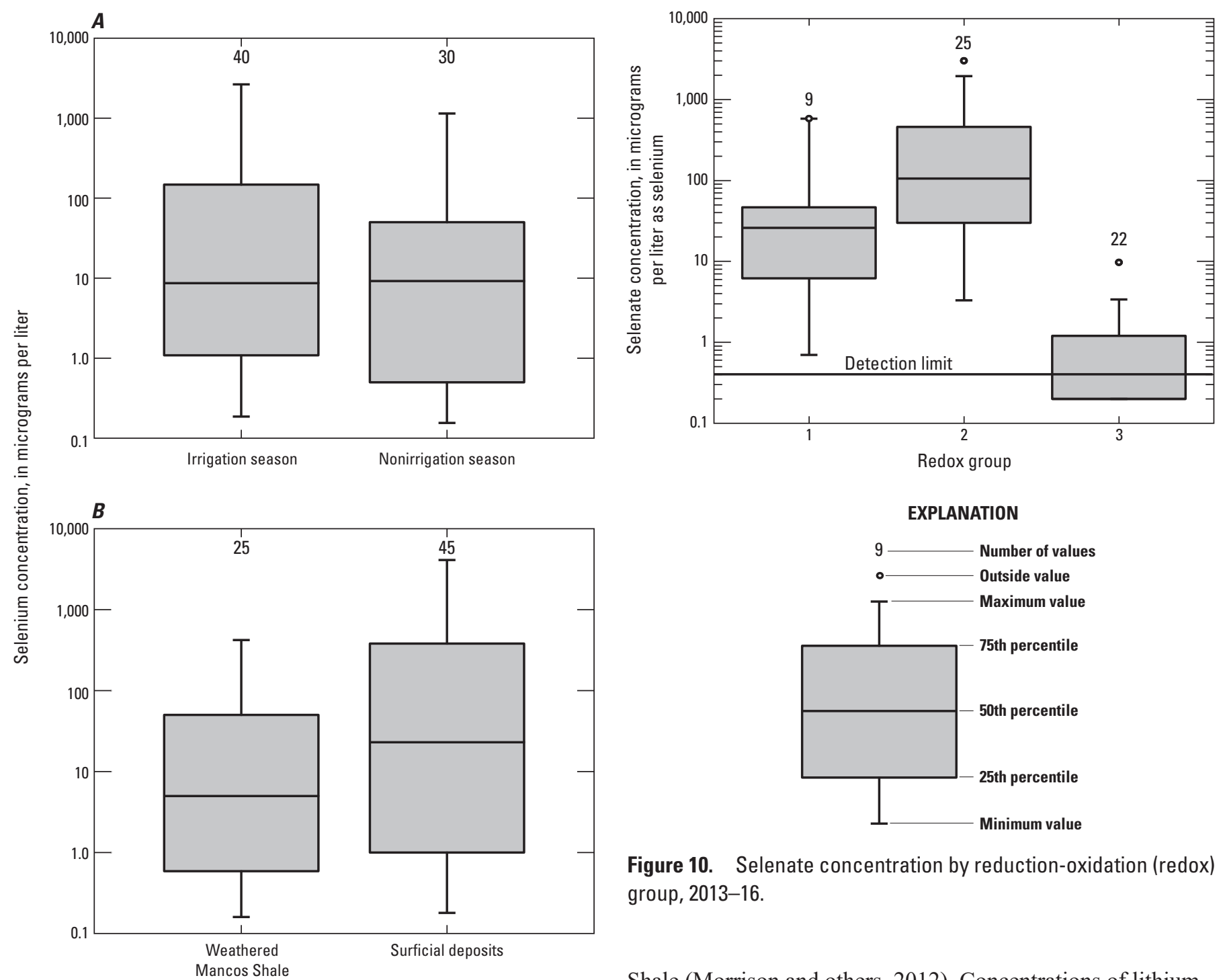

Figure 10. Selenate concentration by reduction-oxidation (redox) group, 2013-16.

Shale (Morrison and others, 2012). Concentrations of lithium, strontium, and uranium were highly correlated with selenium (significant at the $\mathrm{p}<0.01$ level), with $\mathrm{r}$ squared values of $0.75,0.61$, and 0.82 , respectively, supporting the idea that they were sourced from the same material. Streams in the lower Gunnison River Basin have been identified as selenium impaired, and selenium in groundwater is a known contributor to selenium concentrations in surface water as base flow. Similarly, other trace elements were found in high concentrations in groundwater, which enriches surface water with these trace elements. To provide context for the groundwater concentrations of these trace elements, samples were compared to Colorado groundwater agricultural standards (CDPHE, 2016).

High concentrations of boron, iron, manganese,

Figure 9. Selenium concentrations for $A$, the irrigation and nonirrigation seasons and $B$, weathered Mancos Shale and surficial deposits, 2013-16.

lithium, strontium, and uranium (relative to Colorado groundwater agricultural standards [CDPHE, 2016]) were detected in groundwater in the study area (table 9, fig. 11). Trace-element concentrations were not appreciably different between the irrigation and nonirrigation seasons (fig. 11). Concentrations of dissolved boron in groundwater exceeded the Colorado groundwater agricultural standard of $750 \mu \mathrm{g} / \mathrm{L}$ in 33 of 70 samples (47 percent). Concentrations 


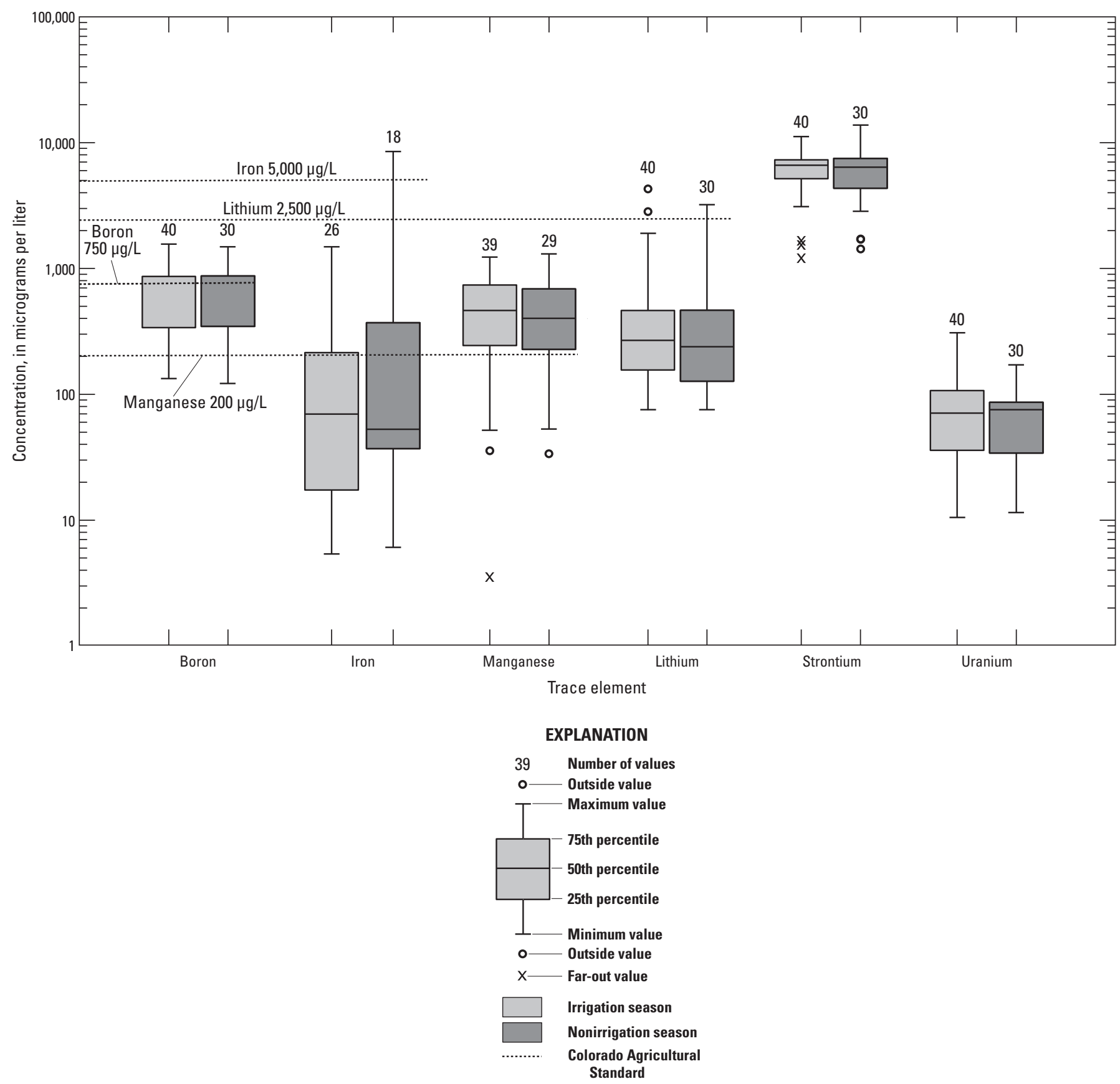

Figure 11. Boron, iron, manganese, lithium, strontium, and uranium concentration during the irrigation and nonirrigation seasons, 2013-16, with Colorado Groundwater Agricultural Standards (Colorado Department of Public Health and Environment, 2016) (standards not available for strontium and uranium). ( $\mu \mathrm{g} / \mathrm{L}$, microgram per liter)

of dissolved iron in groundwater ranged from below the limit of detection to $8,650 \mu \mathrm{g} / \mathrm{L}$ and only exceeded the Colorado groundwater agricultural standard of $5,000 \mu \mathrm{g} / \mathrm{L}$ in one sample (fig. 11). Concentrations of dissolved manganese in groundwater exceeded the Colorado groundwater agricultural standards of 200 standard $\mu \mathrm{g} / \mathrm{L}$ in 54 of 70 samples (77 percent). Concentrations of dissolved lithium in groundwater ranged from 74.9 to $4,860 \mu \mathrm{g} / \mathrm{L}$, with a median concentration of $257 \mu \mathrm{g} / \mathrm{L}$ (table 9, fig. 11). The
Colorado agricultural standard for lithium in groundwater is $2,500 \mu \mathrm{g} / \mathrm{L}$ (CDPHE, 2016), and four lithium concentrations exceeded the agricultural standard. Concentrations of dissolved strontium in groundwater ranged from 1,310 to $15,200 \mu \mathrm{g} / \mathrm{L}$, with a median concentration of $6,700 \mu \mathrm{g} / \mathrm{L}$, and concentrations of dissolved uranium in groundwater ranged from 10.4 to $469 \mu \mathrm{g} / \mathrm{L}$, with a median concentration of $71.1 \mu \mathrm{g} / \mathrm{L}$ (table 9 , fig. 11). No Colorado agricultural groundwater standard exists for strontium or uranium. 


\section{Groundwater Hydrology}

By using data collected as part of this study, the hydrology of the shallow groundwater system can be characterized according to hydraulic conductivity, water level and potentiometric surface, estimates of groundwater recharge, sources of recharge and groundwater ages, denitrification, and estimates of groundwater discharge and selenium loading from groundwater.

\section{Shallow Groundwater System}

The shallow groundwater system would not likely have contained appreciable quantities of water prior to the development of irrigation in the study area, but irrigation now provides substantial quantities of water, allowing for the existence of a shallow groundwater system (Reclamation, 1982; Butler and others, 1996). Of the wells used in this study, 12 were completed in the weathered Mancos Shale and 18 were completed in surficial deposits, which include alluvial gravel, sand, silt, and clay deposited in major river valleys and tributary drainages, in alluvial fans, and as older alluvial terrace, valley fill, or fan deposits (table 10). The 12 wells completed in the weathered Mancos Shale had water-bearing zones in partings in the shale that were typically less than $5 \mathrm{ft}$ thick (Thomas and Arnold, 2015; Thomas, 2015) (fig. 12, table 1). The shallow groundwater system is generally unconfined but is confined or semiconfined where clay sediments predominate or where wells were completed in the weathered Mancos Shale. Wells screened in surficial deposits when confined conditions were observed in the aquifer at the time of the slug test are considered to be semiconfined (table 10).

In the study area, the thickness of surficial deposits generally ranges from 0 to $20 \mathrm{ft}$ where Mancos Shale crops out or has shallow subcrops in upland areas and along mesa slopes and to about $50 \mathrm{ft}$ along segments of the Gunnison River, Uncompahgre River, Loutsenhizer Arroyo, and Montrose Arroyo (fig. 13). Locally, surficial deposits are $<60-80 \mathrm{ft}$ thick along a short segment of Cedar Creek about 6 mi northwest of the City of Montrose, $<80-100 \mathrm{ft}$ thick along an apparent paleovalley of the Uncompahgre River west of the City of Montrose, and up to $140 \mathrm{ft}$ thick along a portion of Uncompahgre River in the southern part of the study area. Gravel deposits on mesas north of the Gunnison River also are up to $140 \mathrm{ft}$ thick (fig. 13). The altitude of the consolidated bedrock surface ranges from about 4,900 ft above NAVD88 in the northwest corner of the study area, near the confluence of the Uncompahgre and Gunnison Rivers, to about $6,700 \mathrm{ft}$ along the southeast edge of the study area (fig. 13). Where surficial deposits are thin, the altitude and configuration of the consolidated bedrock surface underlying surficial deposits was similar to that of the land surface. However, where surficial deposits were thick, the bedrock surface had greater relief between upland areas and valleys. Prominent troughs in the bedrock surface were evident along the Gunnison and Uncompahgre
Rivers as well as along Loutsenhizer Arroyo and some of the larger tributary drainages (fig. 13), indicating that present stream valleys commonly are incised in bedrock. Less prominent troughs in the bedrock surface also were evident along many smaller tributary drainages.

\section{Hydraulic Conductivity}

Results from individual hole-aquifer tests (slug tests) are provided in table 10. Both confined and unconfined conditions were observed in wells during analysis. Hydraulic-conductivity values for surficial deposits ranged from 0.06 to 100 feet per day $(\mathrm{ft} / \mathrm{d})$, with a median of $3.5 \mathrm{ft} / \mathrm{d}$, geometric mean of $3.0 \mathrm{ft} / \mathrm{d}$, and standard deviation of $26 \mathrm{ft} / \mathrm{d}$ (18 wells). Hydraulic-conductivity values for weathered Mancos Shale ranged from 0.3 to $50 \mathrm{ft} / \mathrm{d}$, with a median of $6.0 \mathrm{ft} / \mathrm{d}$, geometric mean of $4.6 \mathrm{ft} / \mathrm{d}$, and standard deviation of $16 \mathrm{ft} / \mathrm{d}$ (12 wells). Conditions in the aquifer at the time of the slug test were found to be unconfined for 14 wells and confined for 16 wells. Of the wells with confined conditions, 4 were screened in the surficial deposits (which make them semiconfined), and the remaining 12 were in the weathered Mancos Shale.

\section{Water Level and Potentiometric Surface}

The potentiometric surface is the level to which water would rise in a tightly cased well at a given point in an aquifer (Lohman, 1972). Where an aquifer is unconfined, the potentiometric surface is defined by the water table. However, where an aquifer is confined by overlying low-permeability sediments, such as clay or shale, groundwater in the aquifer is under pressure, and the potentiometric surface can be above the level of the actual water-bearing sediments or layers. Unless impeded, groundwater flows naturally from areas where the potentiometric surface is high to areas where it is low (Lohman, 1972). Potentiometric surface is represented on a map by potentiometric contours estimating altitude in feet above a land-surface datum.

Depth to water below land surface, as represented by the potentiometric surface, generally was less than $20 \mathrm{ft}$ throughout the study area (table 11). However, water level locally was greater than $20 \mathrm{ft}$ below land surface. As with the bedrock surface, the altitude of the potentiometric surface of the shallow groundwater system ranged from about $4,900 \mathrm{ft}$ near the confluence of the Uncompahgre and Gunnison Rivers to about $6,300 \mathrm{ft}$ along the southeast edge of the study area (fig. 14). Likewise, the potentiometric surface generally reflects the topography of the land surface, with highest altitudes beneath upland areas and mesas. However, the potentiometric surface is generally smoother and has less local relief than the bedrock surface or the land surface.

Where consolidated bedrock occurs at or near the land surface, the altitude of the potentiometric surface might be less than the altitude of the bedrock surface, indicating that the 
Table 10. Estimated hydraulic-conductivity values for monitoring wells, east side of the Uncompahgre River, lower Gunnison River Basin, Colorado.

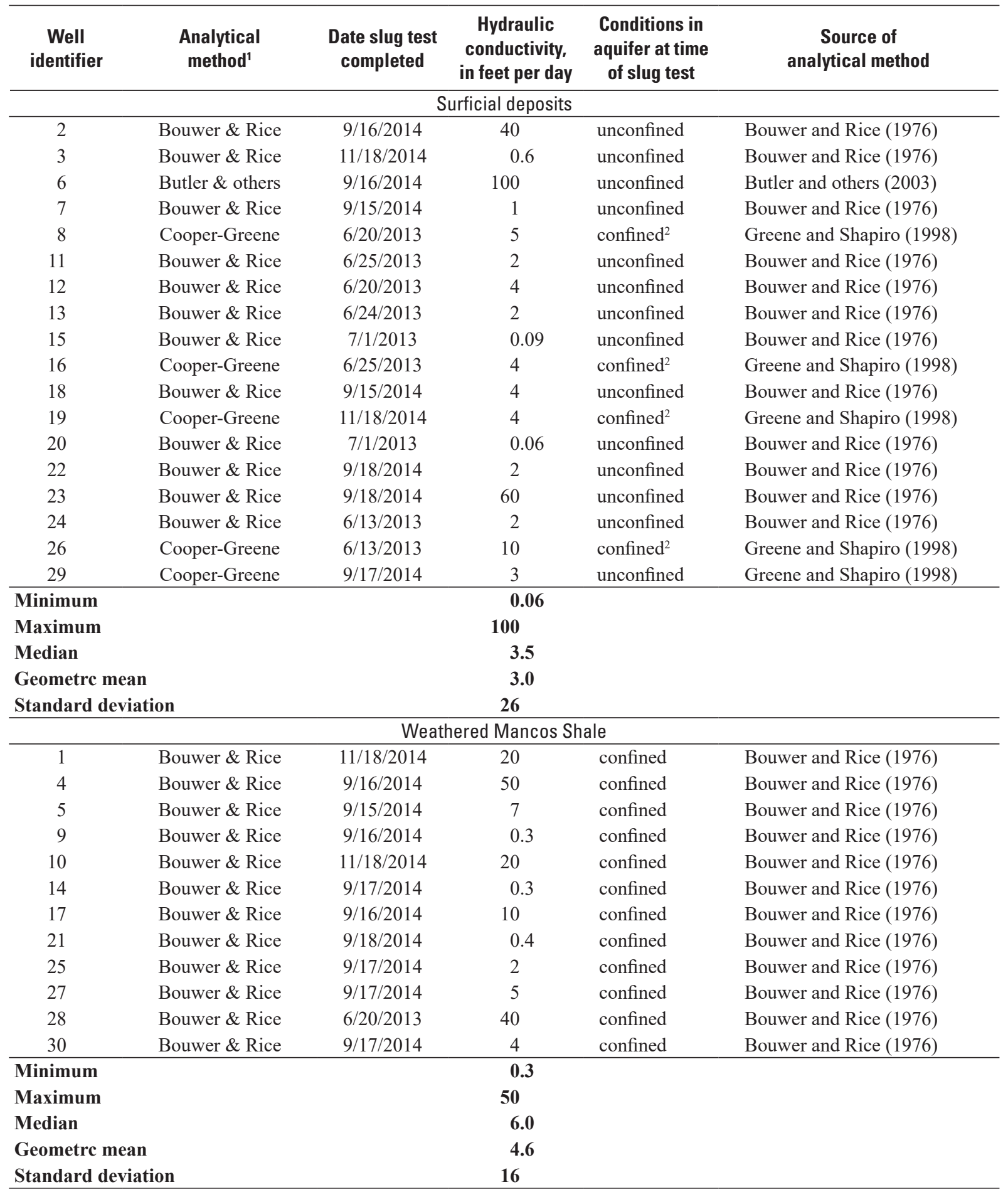

${ }^{1}$ Analytical method selected from the drop-down menu on the "Output" worksheet of the Microsoft Excel spreadsheet file.

${ }^{2} \mathrm{~A}$ well screened in surficial deposits when conditions in the aquifer are confined at the time of the slug test is considered to be semiconfined. 


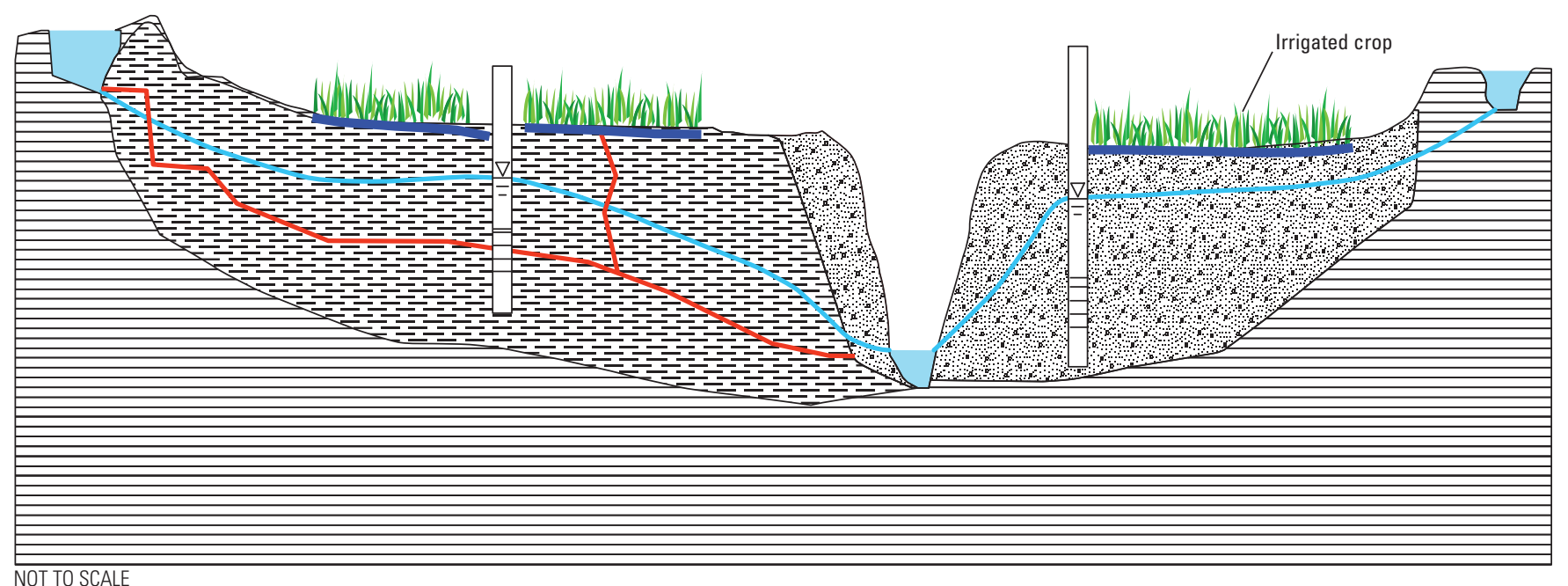

EXPLANATION

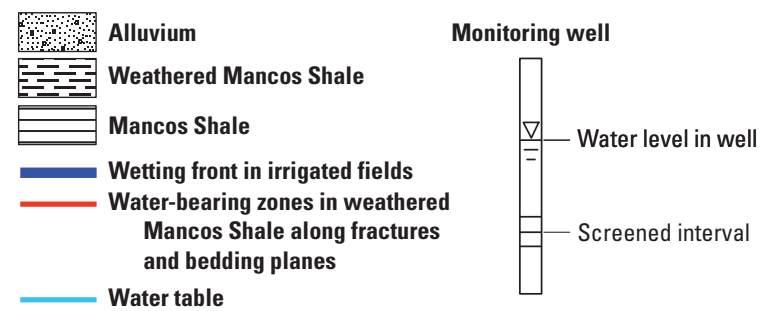

Figure 12. Conceptual cross section of shallow groundwater system showing two scenarios of well completions (figure not to scale).

shallow groundwater system in these areas may be nonexistent or thin, discontinuous, and transient (fig. 14). Although groundwater might not be present in the shallow system at these locations, contours representing the potentiometric surface still are shown in figure 14 to indicate likely directions of flow (if any) through shallow consolidated bedrock and to provide a continuous surface for evaluating potential groundwater flow in the study area.

The direction of groundwater flow in the shallow groundwater system generally was from topographically high areas toward the stream valleys of the Gunnison and Uncompahgre Rivers, Loutsenhizer Arroyo, and large tributary drainages (fig. 14). Groundwater then flows down the stream valleys, with mean gradients generally ranging from about 40 to 60 feet per mile ( $\mathrm{ft} / \mathrm{mi}$ ) (altitude of potentiometric surface over distance on the ground). The mean groundwater gradient along the Gunnison River valley in the study area was about $13 \mathrm{ft} / \mathrm{mi}$ (fig. 14).

A groundwater divide located along the topographic divide between the Uncompahgre River and Loutsenhizer Arroyo (fig. 14), marks where the direction of flow changes between the two stream valleys; groundwater west of the divide flows toward the Uncompahgre River, and groundwater east of the divide flows toward Loutsenhizer Arroyo. The extent of hydraulic connection between groundwater in alluvial upland gravel deposits on mesas and groundwater in alluvial stream, mudflow, and fan deposits along stream valleys is unknown but likely is limited by intervening lowpermeability shale bedrock that crops out or subcrops along the mesa slopes.

Water levels measured during the study period were used to assess seasonal changes in groundwater-level conditions in the study area. Relative change in water level was evaluated by determining the difference of each depth-to-water measurement from the maximum depth to water measured at each well (fig. 15). The maximum depth to water represents the water level that is the greatest distance from the land surface. All wells indicated that water levels respond to the application of irrigation water; response was similar for wells screened in either surficial deposits or the weathered Mancos Shale (fig. 15).

Although irrigation water is typically used from early April to late October, minimum and maximum depths to water varied temporally by well location. The pattern in water levels observed in each well indicated that water-level response to the application of irrigation water was not temporally uniform. Minimum depths to water (water level that is the smallest distance from the land surface) varied between well locations and occurred from May to November; maximum depths to water occurred primarily during April. To illustrate the variability in the timing of the minimum depths to water, depth to water measurements for wells 4 and 25 were plotted together 


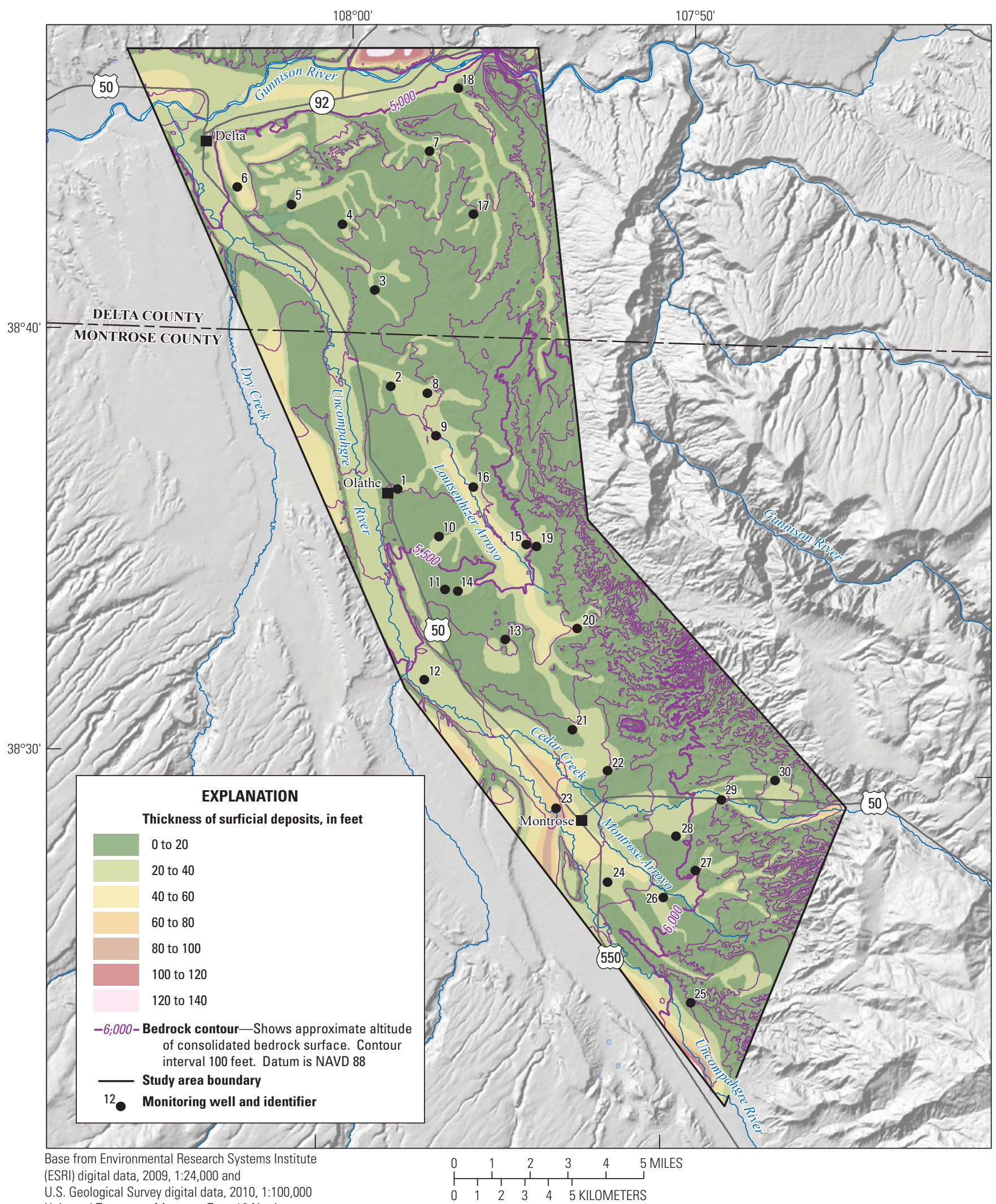

Universal Transverse Mercator, Zone 13 North

North American Datum 1983

Figure 13. Thickness of surficial deposits and altitude of the consolidated bedrock surface, east side of the Uncompahgre River, Colorado. Regolith thickness and bedrock surface modified from Arnold (2017). (NAVD 88, North American Vertical Datum of 1988) 
Table 11. Summary of monthly manual water level measurements including period of record, number of measurements, and minimum and maximum observed water levels for monitoring wells, east side of the Uncompahgre River, lower Gunnison River Basin, Colorado.

[ft, foot; LSD, land surface datum; NAVD 1988, North American Vertical Datum of 1988. Gray shading indicates sites with continuous groundwater-level data]

\begin{tabular}{|c|c|c|c|c|c|c|c|c|c|}
\hline $\begin{array}{c}\text { Well } \\
\text { identifier }\end{array}$ & $\begin{array}{c}\text { Altitude of } \\
\text { LSD } \\
\text { (ft above } \\
\text { NAVD88) }\end{array}$ & $\begin{array}{l}\text { Begin date of } \\
\text { depth-to-water } \\
\text { measurements }\end{array}$ & $\begin{array}{c}\text { End date of } \\
\text { depth-to-water } \\
\text { measurements }\end{array}$ & $\begin{array}{l}\text { Total number of } \\
\text { depth-to-water } \\
\text { measurements }\end{array}$ & $\begin{array}{c}\text { Minimum } \\
\text { depth-to-water, } \\
\text { ft below LSD }\end{array}$ & $\begin{array}{c}\text { Maximum } \\
\text { depth-to-water, } \\
\text { ft below LSD' }\end{array}$ & $\begin{array}{c}\text { Minimum } \\
\text { water-level } \\
\text { altitude above } \\
\text { NAVD 1988, ft }\end{array}$ & $\begin{array}{c}\text { Maximum } \\
\text { water-level } \\
\text { altitude above } \\
\text { NAVD 1988, } \mathrm{ft}^{2}\end{array}$ & $\begin{array}{c}\text { Range in } \\
\text { depth to water, } \\
\text { ft below LSD }\end{array}$ \\
\hline 1 & 5,416 & $4 / 26 / 2014$ & $11 / 16 / 2016$ & 27 & 7.39 & 9.08 & 5,407 & 5,409 & 1.7 \\
\hline 2 & 5,286 & $4 / 15 / 2014$ & $9 / 20 / 2016$ & 22 & 4.62 & 9.31 & 5,277 & 5,281 & 4.7 \\
\hline 3 & 5,245 & $4 / 12 / 2014$ & $11 / 16 / 2016$ & 26 & 1.09 & 3.98 & 5,241 & 5,244 & 2.9 \\
\hline 4 & 5,190 & $4 / 24 / 2014$ & $11 / 16 / 2016$ & 28 & 5.7 & 11.6 & 5,178 & 5,184 & 5.9 \\
\hline 5 & 5,164 & $4 / 24 / 2014$ & $11 / 16 / 2016$ & 24 & 11.89 & 20.4 & 5,144 & 5,152 & 8.5 \\
\hline 6 & 5,094 & 6/9/2014 & $11 / 16 / 2016$ & 24 & 28.4 & 37.28 & 5,057 & 5,066 & 8.9 \\
\hline 7 & 5,087 & $4 / 23 / 2014$ & $11 / 16 / 2016$ & 24 & 16.86 & 20.5 & 5,067 & 5,070 & 3.6 \\
\hline 8 & 5,302 & $11 / 11 / 2012$ & $11 / 16 / 2016$ & 24 & 10.09 & 14.37 & 5,288 & 5,292 & 4.3 \\
\hline 9 & 5,364 & $4 / 25 / 2014$ & $11 / 16 / 2016$ & 23 & 8.35 & 12.03 & 5,352 & 5,356 & 3.7 \\
\hline 10 & 5,483 & $4 / 12 / 2014$ & $11 / 16 / 2016$ & 23 & 7.93 & 14.82 & 5,468 & 5,475 & 6.9 \\
\hline 11 & 5,559 & $11 / 14 / 2012$ & $11 / 16 / 2016$ & 25 & 2.31 & 6.95 & 5,552 & 5,557 & 4.6 \\
\hline 12 & 5,544 & $11 / 11 / 2012$ & $11 / 16 / 2016$ & 26 & 3.17 & 9.53 & 5,534 & 5,541 & 6.4 \\
\hline 13 & 5,604 & $11 / 14 / 2012$ & $11 / 16 / 2016$ & 27 & 7.05 & 8.59 & 5,595 & 5,597 & 1.5 \\
\hline 14 & 5,576 & $4 / 28 / 2014$ & $11 / 16 / 2016$ & 24 & 15.62 & 22.2 & 5,554 & 5,560 & 6.6 \\
\hline 15 & 5,506 & $10 / 29 / 2012$ & $11 / 16 / 2016$ & 25 & 4.5 & 8.02 & 5,498 & 5,502 & 3.5 \\
\hline 16 & 5,409 & $10 / 29 / 2012$ & $11 / 16 / 2016$ & 32 & 19.53 & 27.72 & 5,381 & 5,389 & 8.2 \\
\hline 17 & 5,227 & $4 / 12 / 2014$ & $11 / 16 / 2016$ & 23 & -1.26 & 8.6 & 5,218 & 5,228 & 9.9 \\
\hline 18 & 5,060 & $4 / 23 / 2014$ & $11 / 16 / 2016$ & 24 & 5.98 & 14.8 & 5,045 & 5,054 & 8.8 \\
\hline 19 & 5,531 & $4 / 26 / 2014$ & $11 / 16 / 2016$ & 24 & 1.47 & 7.5 & 5,524 & 5,530 & 6.0 \\
\hline 20 & 5,657 & $10 / 29 / 2012$ & $11 / 16 / 2016$ & 29 & 0.41 & 2.51 & 5,654 & 5,657 & 2.1 \\
\hline 21 & 5,743 & $4 / 29 / 2014$ & $11 / 16 / 2016$ & 30 & 5.08 & 14.55 & 5,728 & 5,738 & 9.5 \\
\hline 22 & 5,814 & $4 / 14 / 2014$ & $11 / 16 / 2016$ & 25 & 4.19 & 6.26 & 5,808 & 5,810 & 2.1 \\
\hline 23 & 5,762 & 6/9/2014 & $11 / 16 / 2016$ & 24 & 8.1 & 9.8 & 5,752 & 5,754 & 1.7 \\
\hline 24 & 5,876 & $10 / 29 / 2012$ & $11 / 16 / 2016$ & 27 & 2.02 & 4.94 & 5,871 & 5,874 & 2.9 \\
\hline 25 & 6,121 & $4 / 28 / 2014$ & $11 / 16 / 2016$ & 30 & 5.64 & 9.8 & 6,111 & 6,115 & 4.2 \\
\hline 26 & 5,954 & $10 / 29 / 2012$ & $11 / 16 / 2016$ & 31 & 0.99 & 8.34 & 5,946 & 5,953 & 7.4 \\
\hline 27 & 6,021 & $4 / 10 / 2014$ & $11 / 16 / 2016$ & 26 & -0.49 & 2.31 & 6,019 & 6,021 & 2.8 \\
\hline 28 & 5,995 & $10 / 29 / 2012$ & $11 / 16 / 2016$ & 27 & 7.14 & 14.47 & 5,981 & 5,988 & 7.3 \\
\hline 29 & 6,106 & $4 / 13 / 2014$ & $11 / 16 / 2016$ & 30 & 0.75 & 7.8 & 6,098 & 6,105 & 7.1 \\
\hline 30 & 6,247 & $4 / 16 / 2014$ & $11 / 16 / 2016$ & 30 & 0.94 & 9.15 & 6,238 & 6,246 & 8.2 \\
\hline
\end{tabular}

${ }^{1}$ Maximum depth to water level represents the water level that is the greatest distance from the land surface.

${ }^{2}$ Maximum water-level altitude represents the water-level altitude that is the greatest distance from the land-surface datum, NAVD 1988. 


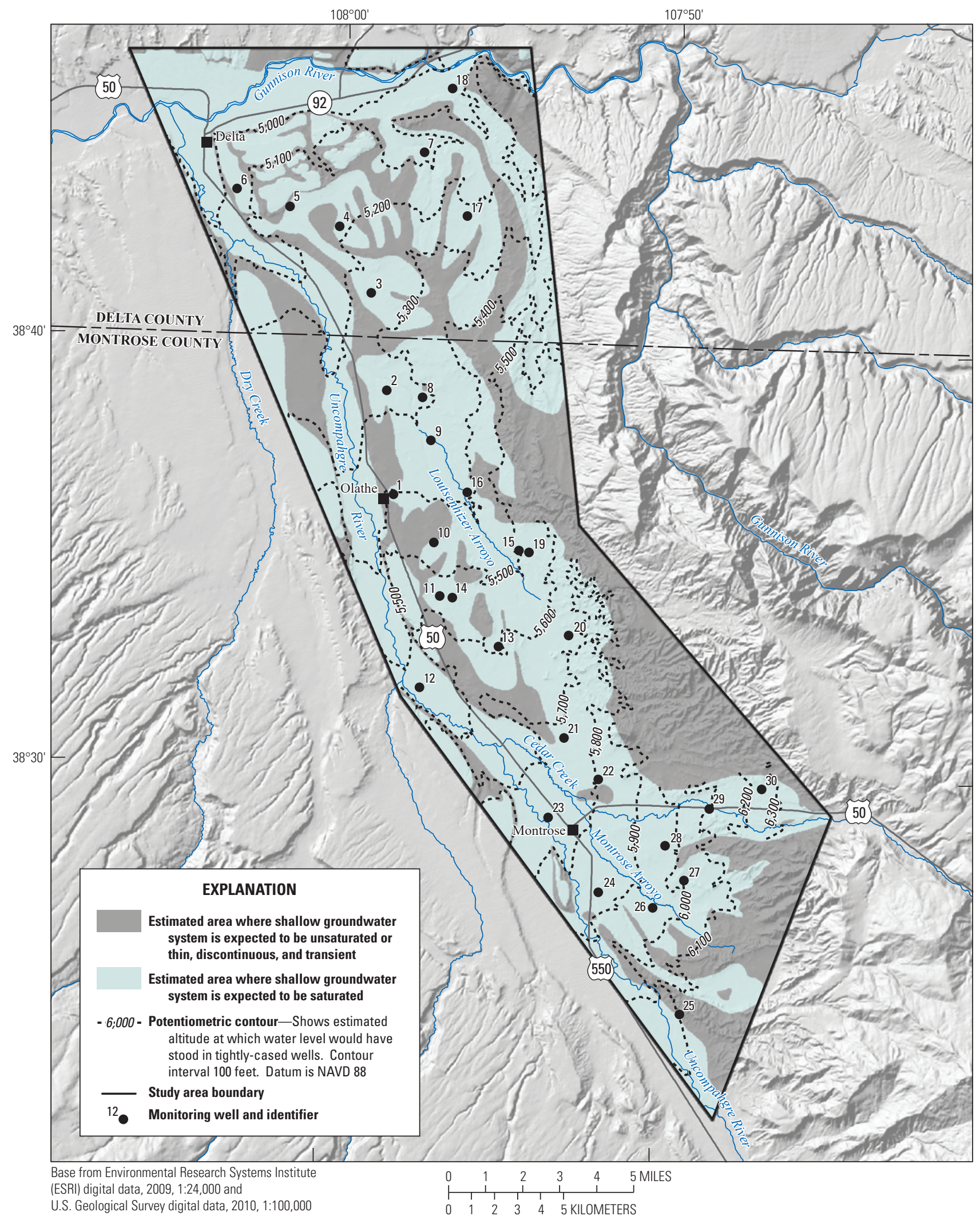

U.S. Geological Survey digital data, 2010, 1:100,000 North American Datum 1983

Figure 14. Estimated altitude of the potentiometric contours, based on water levels measured during 2012-16; areas where the shallow groundwater system is expected to be unsaturated or thin, discontinuous, or transient; and estimated areas where the shallow groundwater system is expected to be saturated, east side of the Uncompahgre River, Colorado. Regolith thickness and bedrock surface modified from Arnold (2017). (NAVD 88, North American Vertical Datum of 1988) 


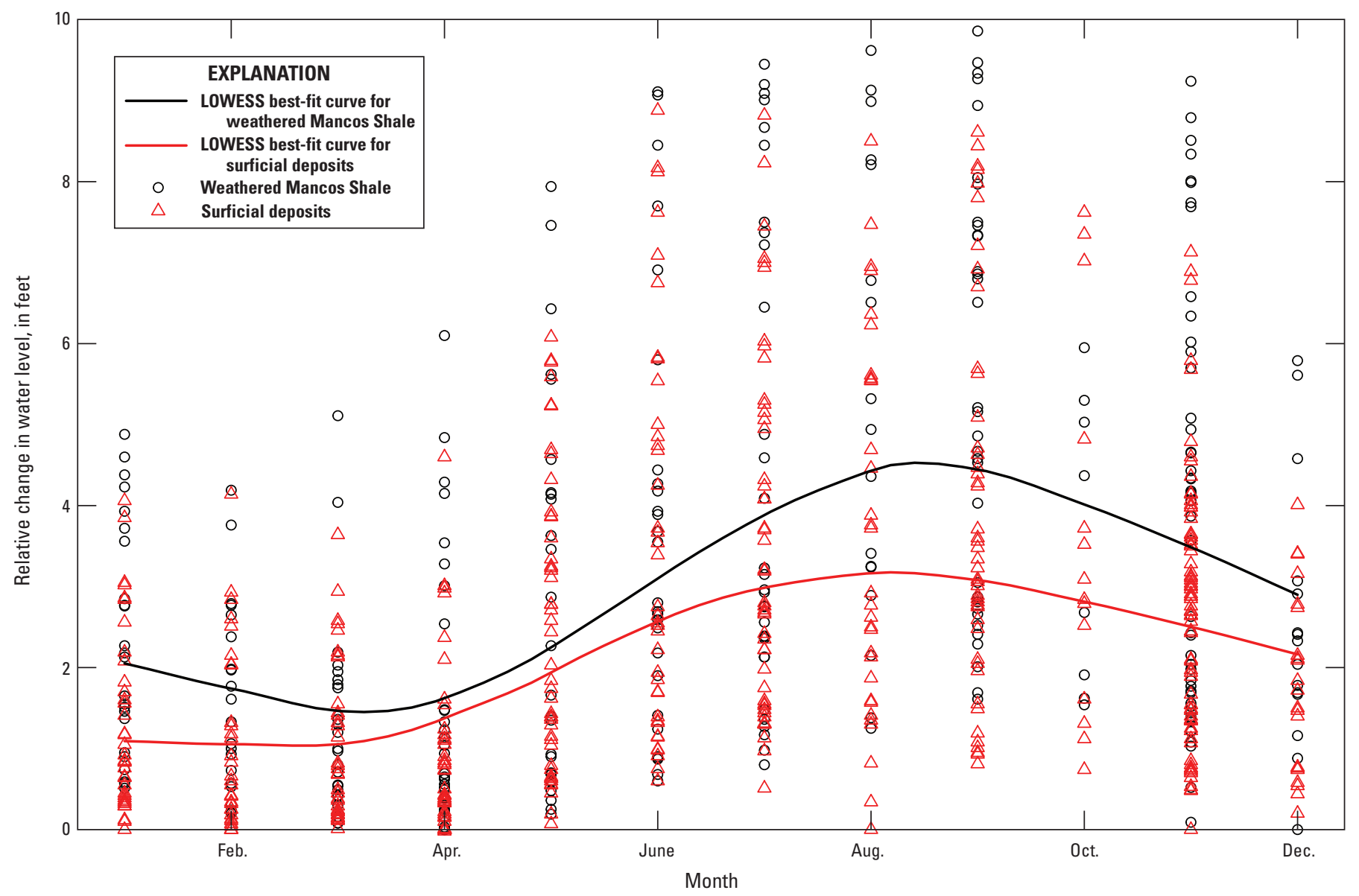

Figure 15. Seasonal change in water levels in monitoring wells, 2014-16. Values of relative change in water level are represented by the difference of each depth-to-water measurement from the maximum depth to water measured at each well, and the solid lines represent a LOWESS (Cleveland, 1981) best-fit curve of the values.

(fig. 16); minimum depths to water for well 4 occurred during October or November, and minimum depths to water for well 25 occurred during April or May (fig. 16). The variability in the timing of minimum depths to water between wells may indicate differences in rates of recharge or distances of wells from recharge sources.

\section{Estimates of Groundwater Recharge}

Recharge to the shallow groundwater system occurs primarily during the irrigation season as water fills canals and is applied to fields, with little recharge from precipitation. Mean annual precipitation in Delta, Colo., for 1900-99 is 7.92 inches per year (in/yr) (Western Regional Climate Center, 2017), and mean annual precipitation in Montrose, Colo., for 1895-2016 is $9.60 \mathrm{in} / \mathrm{yr}$ (Western Regional Climate Center, 2017). Given the general absence of saturated sediments in nonirrigated parts of the study area, it is likely that little of this precipitation recharged the shallow groundwater system. Increasingly in the study area, formerly agricultural land is being converted to urban and residential land uses (Gunnison
Basin Selenium Task Force, 2017), resulting in a decrease in the amount of irrigated acreage. This decrease in irrigation would likely have an effect on the source and amount of recharge to the shallow groundwater system.

The amount of recharge to the unconfined portion of the shallow groundwater system was estimated by using the graphical approach to the water-table fluctuation (WTF) method, in which the antecedent recession curves were extrapolated manually to obtain the peak water-table rise (Delin and others, 2007) (appendix 1). Specific yield (Sy) values were estimated from knowledge of the lithologic material for each well and compared to values derived by Johnson (1967). A minimum and maximum Sy value was used for each well (table 12). Estimated recharge values range from 0.0 to $37.8 \mathrm{in} / \mathrm{yr}$ (table 12) with a mean of $9.4 \mathrm{in} / \mathrm{yr}$. The minimum recharge value was observed at well 7 , where the geologic materials are unconsolidated clay with trace amounts of sand (Thomas, 2015). Well 7 is located on the west side of a natural drain adjacent to residential land use; the area directly surrounding well 7 was not irrigated, but well 7 is downgradient of irrigated land (fig. 3). The maximum recharge value was observed at well 6 , where 


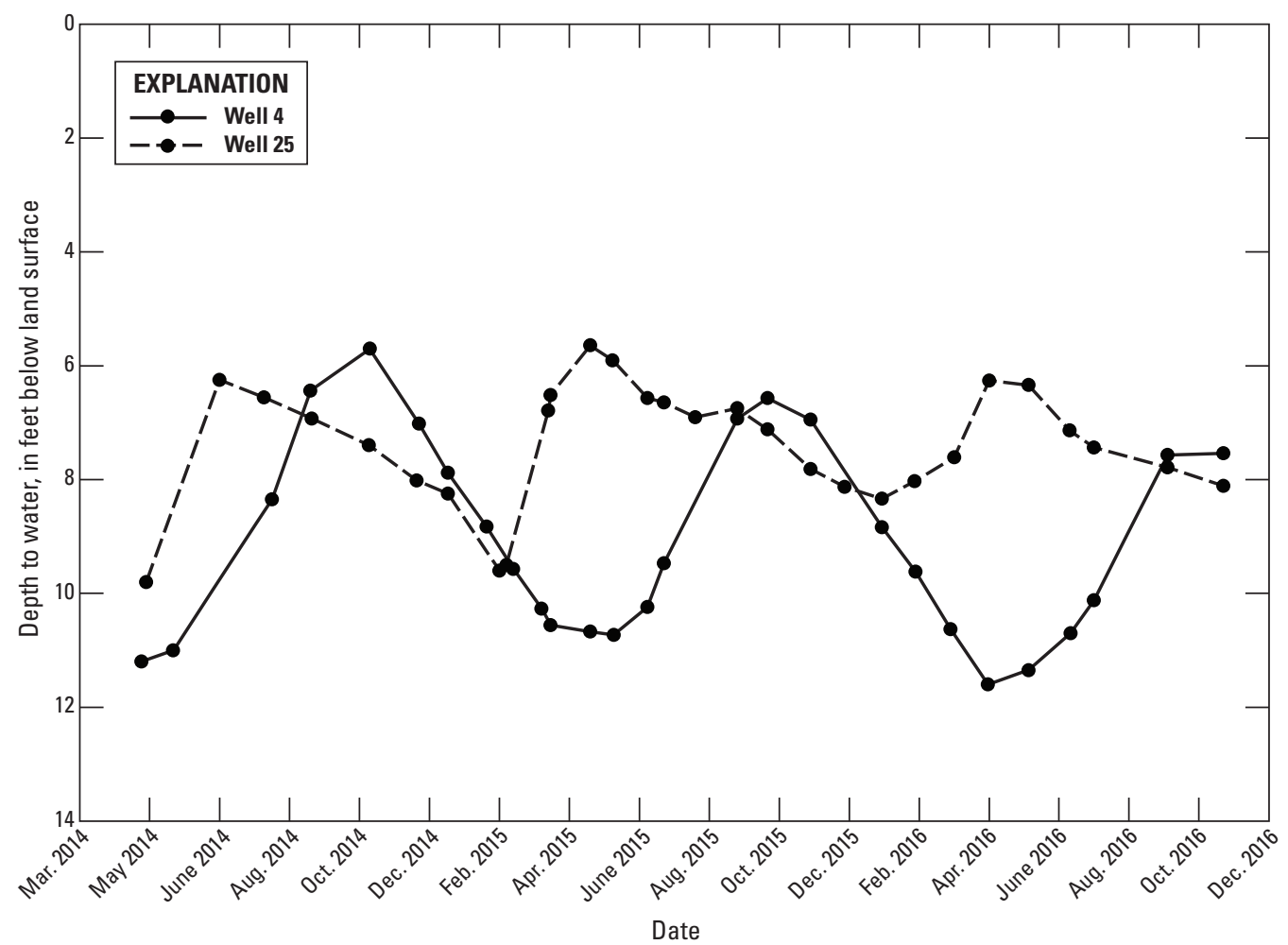

Figure 16. Depths to water measurements for wells 4 and 25 for April 2014-November 2016. Both wells were completed in the weathered Mancos Shale and exhibit confined conditions.

the geologic materials consist of poorly sorted cobbles and gravel with trace clay (Thomas, 2015). Well 6 is located in an irrigated field near residential land use (fig. 3).

Recharge estimates from table 12 represent the amount of recharge to the unconfined groundwater system based on changes in water level and Sy. Mean annual precipitation in the study area ranges from 7.9 to $9.6 \mathrm{in} / \mathrm{yr}$. Although the mean recharge values for the unconfined aquifer in the study area were similar to mean annual precipitation, mean recharge at individual wells was as much as three times that of precipitation, indicating that infiltration by precipitation is not the only source of recharge. Previous investigations estimated that the amount of irrigation water applied to fields in the lower Gunnison River Basin ranged from 40 to 84 in. (3.3 to $7 \mathrm{ft}$ ) depending on land use, irrigation method, and crop type (Linard, 2013). A study conducted in the Grand Valley estimated a mean value of 1.27 acre-feet of the 3.79 acre-feet of irrigation applied (around 30 percent) was deep percolating (Mayo, 2008). Recharge values determined by using the WTF method similarly indicated that as much as 30 percent of the irrigation applied is recharging the shallow groundwater system. However, because the WTF method is best suited for unconfined aquifers, estimating the amount of recharge to the confined aquifer through an approach similar to that of the unconfined aquifer was less straightforward and would not yield representative values of recharge. Estimating groundwater recharge to the confined aquifer could involve the use of a groundwater model or acquiring additional data, which was beyond the scope of the study. Potential sources of recharge to the confined aquifer are likely a combination of canal leakage, in which water moves from the canal along bedding planes and vertical fractures that provide a pathway through the shale down to a bedding plane where water is being conveyed.

\section{Sources of Recharge and Groundwater Age}

The stable isotopic composition of groundwater was used to help determine sources of recharge to the shallow groundwater system and to determine whether processes like evaporation affected recharge (fig. 17). Isotopic values were determined for groundwater samples collected during both the irrigation and nonirrigation seasons (fig. 17). In addition, water isotopes were determined for four surface-water sources and a deep groundwater source. Two surface-water sources were selected at the beginning of the irrigation systemUncompahgre River at Colona (USGS station identifier [ID] 09147500) and South Canal at Uncompahgre Road (USGS ID 382241107480401) - and two were selected closer to the end of the irrigation system-East Canal Tail Water into Sunflower Drain (USGS ID 384200107573901) and Sunflower 
Table 12. Estimated recharge based on the water table fluctuation (WTF) method for unconfined wells, Delta and Montrose Counties, Colorado.

[Graphs for each well located in appendix 1]

\begin{tabular}{|c|c|c|c|c|c|c|}
\hline $\begin{array}{l}\text { Well } \\
\text { identifier }\end{array}$ & $\begin{array}{l}\text { Water-level } \\
\text { rise, feet }\end{array}$ & $\begin{array}{c}\text { Specific yield, } \\
\text { minimum }\end{array}$ & $\begin{array}{l}\text { Specific yield, } \\
\text { maximum }\end{array}$ & $\begin{array}{l}\text { WTF minimum } \\
\text { recharge, } \\
\text { inches per year }\end{array}$ & $\begin{array}{l}\text { WTF maximum } \\
\text { recharge, } \\
\text { inches per year }\end{array}$ & $\begin{array}{l}\text { WTF mean- } \\
\text { recharge, } \\
\text { inches per year }\end{array}$ \\
\hline 2 & 4.3 & 0.15 & 0.32 & 7.7 & 16.5 & 12.1 \\
\hline 2 & 2.0 & 0.15 & 0.32 & 3.6 & 7.7 & 5.6 \\
\hline 3 & 1.7 & 0.03 & 0.12 & 0.59 & 2.4 & 1.5 \\
\hline 6 & 9.0 & 0.20 & 0.35 & 21.6 & 37.8 & 29.7 \\
\hline 6 & 8.5 & 0.20 & 0.35 & 20.4 & 35.7 & 28.1 \\
\hline 7 & 1.0 & 0.0 & 0.05 & 0.0 & 0.6 & 0.30 \\
\hline 7 & 0.6 & 0.0 & 0.05 & 0.0 & 0.4 & 0.18 \\
\hline 11 & 1.2 & 0.1 & 0.28 & 1.4 & 4.0 & 2.7 \\
\hline 11 & 1.7 & 0.1 & 0.28 & 2.0 & 5.7 & 3.9 \\
\hline 12 & 6.5 & 0.13 & 0.26 & 10.1 & 20.3 & 15.2 \\
\hline 12 & 5.5 & 0.13 & 0.26 & 8.6 & 17.2 & 12.9 \\
\hline 13 & 1.5 & 0.13 & 0.26 & 2.3 & 4.5 & 3.4 \\
\hline 13 & 1.2 & 0.13 & 0.26 & 1.8 & 3.6 & 2.7 \\
\hline 15 & 3.8 & 0.0 & 0.05 & 0.0 & 2.3 & 1.1 \\
\hline 15 & 3.5 & 0.0 & 0.05 & 0.0 & 2.1 & 1.1 \\
\hline 18 & 8.3 & 0.20 & 0.35 & 19.8 & 34.7 & 27.2 \\
\hline 18 & 8.1 & 0.20 & 0.35 & 19.4 & 34.0 & 26.7 \\
\hline 20 & 2.4 & 0.0 & 0.05 & 0.0 & 1.4 & 0.72 \\
\hline 20 & 1.9 & 0.0 & 0.05 & 0.0 & 1.1 & 0.56 \\
\hline 22 & 2.4 & 0.15 & 0.32 & 4.2 & 9.0 & 6.6 \\
\hline 22 & 1.8 & 0.15 & 0.32 & 3.2 & 6.7 & 4.9 \\
\hline 23 & 1.6 & 0.20 & 0.35 & 3.8 & 6.7 & 5.3 \\
\hline 23 & 1.4 & 0.20 & 0.35 & 3.2 & 5.7 & 4.5 \\
\hline 24 & 3.0 & 0.20 & 0.35 & 7.2 & 12.6 & 9.9 \\
\hline 29 & 7.3 & 0.15 & 0.32 & 13.1 & 27.8 & 20.4 \\
\hline \multirow[t]{2}{*}{29} & 6.5 & 0.15 & 0.32 & 11.7 & 25.0 & 18.3 \\
\hline & & & & & Mean & 9.4 \\
\hline
\end{tabular}

Drain at Highway 92 (USGS ID 384551107591901 ) (fig. 17). The selected deep groundwater source-Iron Mike (USGS ID 382846107523001) - is a flowing artesian well located in Montrose that was drilled in the 1800 s and completed in the Dakota Sandstone (fig. 17).

Isotopic values for surface-water samples plotted near the local meteoric water line (LMWL) defined by Coplen and Kendall (2000) through data collected from the Gunnison River near Grand Junction; this correlation indicates that water was derived from precipitation (fig. 17). Values from the deep groundwater sample plotted below the LMWL and had the most negative isotopic values in the dataset.

Assuming relatively little temporal variability in the isotopic composition of the deep groundwater, the data indicate deep groundwater was not an important source of recharge to the shallow groundwater system. Most of the other groundwater samples plotted parallel to the LMWL, but generally there was a small positive shift in $\delta^{18} \mathrm{O}$ as compared to the surfacewater samples. Small shifts in the groundwater data relative to the surface-water data may be due, in part, to the small number of surface-water samples that were analyzed for the water isotopes; they may not capture the full range of isotopic variability in canal water used to irrigate fields. Larger positive shifts in $\delta^{18} \mathrm{O}$ in the groundwater data relative to the LMWL could indicate that groundwater was affected by evaporation before or after recharge. Data from wells 3 and 7 deviated farthest from the LMWL (fig. 17). Lines extending from data points for those wells back to the surface-water samples had slopes from about 4 to 5 , consistent with slopes of 3 to 6 commonly associated with water affected by evaporation (Coplen 


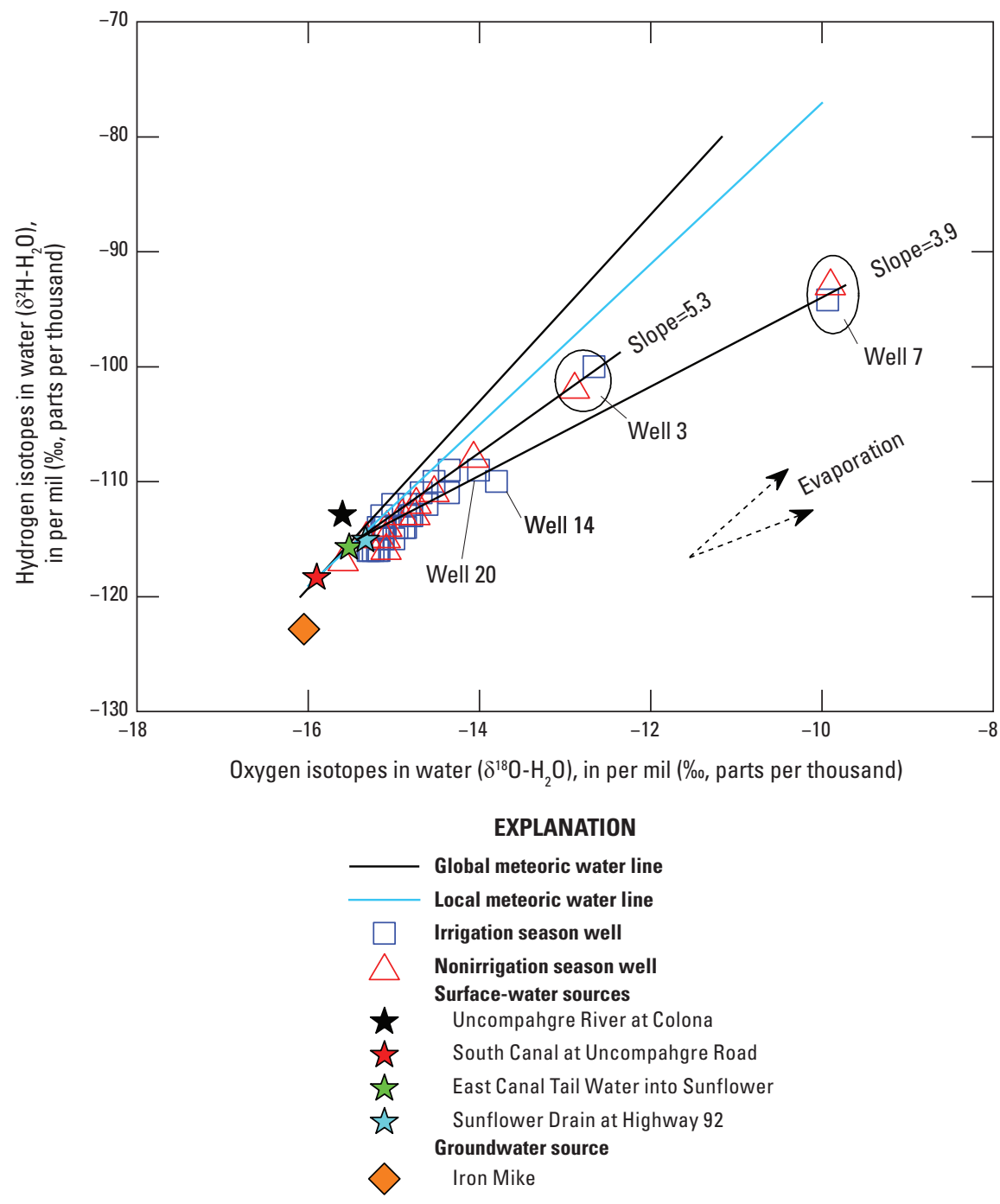

Figure 17. Stable isotopic composition of groundwater and surface water from the east side of the Uncompahgre River, lower Gunnison River Basin, Colorado, 2014-15 and local and global meteoric water lines. Local meteoric water line from Coplen and Kendall (2000).

and others, 2000). Despite the apparent effects of evaporation on some of the samples, the isotopic data are consistent with water diverted from local rivers and reservoirs being the primary source of recharge to the shallow groundwater system.

Tritium concentrations in groundwater collected during the nonirrigation season in 2015 ranged from 0.91 to 5.72 tritium units (TU) (table 13). Water that recharged the shallow groundwater system in the study area before the early 1950s contained approximately 7 to $8 \mathrm{TU}$ at the time of recharge (Thatcher, 1962; Michel, 1989), but recharge water sampled in 2015 would contain less than $0.3 \mathrm{TU}$ because of radioactive decay of the tritium. These data indicate that all samples analyzed for tritium contained at least some groundwater that had been recharged since the early 1950s (post-1950s recharge).

Mean groundwater ages were computed from the tritium, ${ }^{3} \mathrm{He}_{\text {trit }}$, and $\mathrm{SF}_{6}$ data to refine the understanding of groundwater residence times in the shallow groundwater system (Jurgens and others, 2012). Because ${ }^{3} \mathrm{He}_{\text {trit }}$ and $\mathrm{SF}_{6}$ are normally present as dissolved gases in groundwater, they are susceptible to being stripped from solution in samples that are actively degassing because of high gas contents. As a result, actively degassing samples were unsuitable for groundwater age dating. Groundwater degassing was observed during sampling of some of the wells; carbon dioxide was most likely the gas observed, given its high concentration relative to other gases in many of the samples (table 8; fig. 18). Data on $\mathrm{N}_{2}$ gas and argon gas were used to identify which samples were most affected by gas stripping and therefore could not be used for age dating (fig. 18). Concentrations of $\mathrm{N}_{2}$ and argon in many of the samples plotted near the air-saturated water line at recharge temperatures between about 10 and 15 degrees Celsius $\left({ }^{\circ} \mathrm{C}\right)$ or along trajectories for excess air that started at those temperatures (fig. 18). It is common for excess air to be trapped in groundwater at the time of 
Table 13. Tritium data for water collected from monitoring wells during the nonirrigation season in 2015, east side of the Uncompahgre River, lower Gunnison River Basin, Colorado.

[TU, tritium unit]

\begin{tabular}{cccc}
\hline $\begin{array}{c}\text { Well } \\
\text { identifier }\end{array}$ & $\begin{array}{c}\text { Collection } \\
\text { date }\end{array}$ & $\begin{array}{c}\text { Tritium, } \\
\text { water, } \\
\text { unfiltered (TU) }\end{array}$ & $\begin{array}{c}\text { Tritium, water, } \\
\text { 1 standard } \\
\text { deviation error } \\
\text { (TU) }\end{array}$ \\
\hline 2 & $3 / 5 / 2015$ & 4.66 & 0.19 \\
3 & $3 / 5 / 2015$ & 4.35 & 0.24 \\
6 & $3 / 12 / 2015$ & 4.89 & 0.17 \\
7 & $3 / 6 / 2015$ & 0.91 & 0.12 \\
8 & $3 / 5 / 2015$ & 5.32 & 0.22 \\
9 & $3 / 10 / 2015$ & 4.12 & 0.13 \\
10 & $3 / 11 / 2015$ & 4.39 & 0.19 \\
15 & $3 / 9 / 2015$ & 3.99 & 0.27 \\
17 & $3 / 6 / 2015$ & 5.34 & 0.35 \\
18 & $3 / 10 / 2015$ & 5.72 & 0.32 \\
22 & $3 / 4 / 2015$ & 4.19 & 0.13 \\
25 & $3 / 3 / 2015$ & 5.32 & 0.27 \\
\hline
\end{tabular}
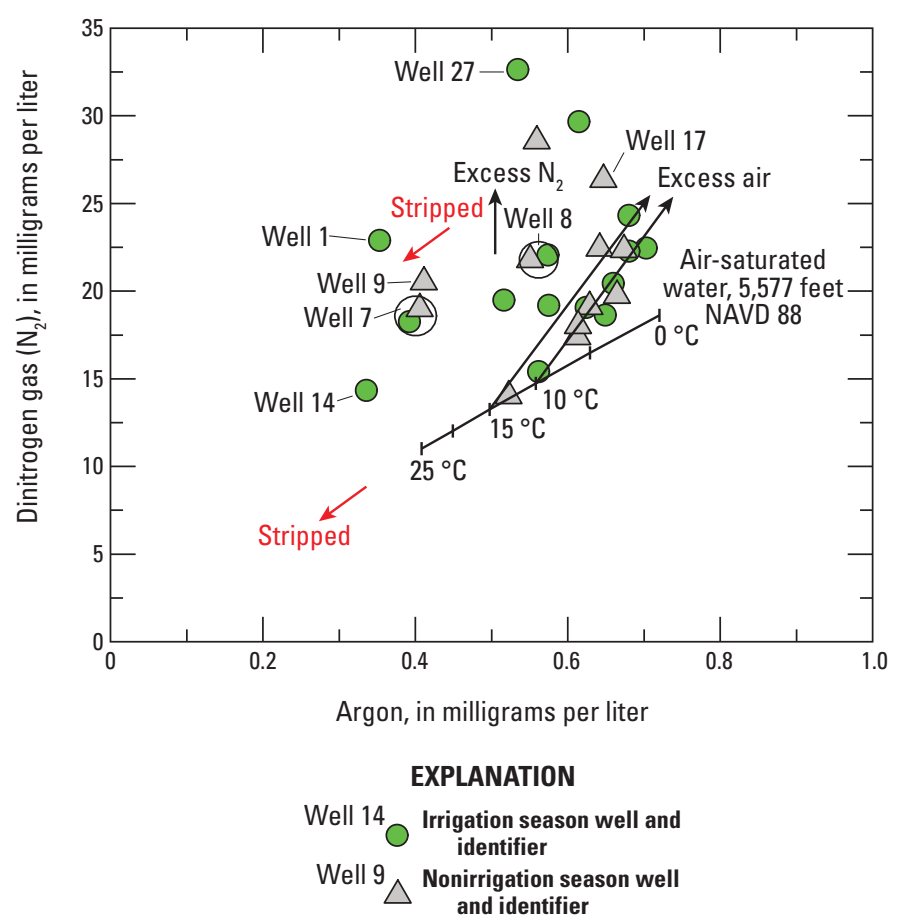

NAVD 88 North American Vertical Datum of 1988

Figure 18. Data on dissolved-gas concentrations (dinitrogen and argon) in groundwater collected from monitoring wells during the irrigation and nonirrigation seasons, 2014-15, east side of the Uncompahgre River, lower Gunnison River Basin, Colorado.

recharge (Heaton and Vogel, 1981). Those samples showed no evidence of gas stripping, so their ${ }^{3} \mathrm{He}_{\text {trit }}$ and $\mathrm{SF}_{6}$ data could potentially be used for age dating. Several samples (for example, from wells 8,17 , and 27) plotted above the excessair trajectories, indicating they probably contained excess $\mathrm{N}_{2}$ from denitrification (Böhlke and Denver, 1995). The ${ }^{3} \mathrm{He}_{\text {trit }}$ and $\mathrm{SF}_{6}$ in those samples could also potentially be used for age dating. At least four samples (from wells 1, 7, 9, and 14) appeared to contain excess $\mathrm{N}_{2}$ and to be affected by gas stripping. The ${ }^{3} \mathrm{He}_{\text {trit }}$ and $\mathrm{SF}_{6}$ in those samples were not used for age dating. The other factor that could affect the utility of $\mathrm{SF}_{6}$ as an age tracer is $\mathrm{SF}_{6}$ contamination in groundwater from natural or human sources (Busenberg and Plummer, 2000). Samples from wells 5, 10, 18, and 24 were the only samples analyzed for $\mathrm{SF}_{6}$ that were not affected by contamination as determined by the analyzing laboratory. The source of the contamination is unknown.

Seven samples, each from a different well, had tracer data suitable for age dating. One of the samples (from well 5) was collected during the 2014 irrigation season and the remaining six samples were collected during the 2015 nonirrigation season (table 14). Groundwater samples can contain fractions of water of various ages. The distribution of ages in groundwater samples may take various forms depending on factors such as aquifer thickness and depth and length of well screens (Jurgens and others, 2012). The tracer data appeared to fit groundwater-age distributions represented by exponentialmixing, dispersion models, or both (fig. 19) (Jurgens and others, 2012). Mean groundwater ages in the samples based on these models range from 6 to 20 years and correspond to recharge years ranging from 1995 to 2009 (table 14, fig. 20). Four of the six samples collected during the nonirrigation season appear to have been recharged since 2005 (table 14). The only sample with a recharge year before 2000 was collected during the nonirrigation season (table 14). The number of samples from the irrigation and nonirrigation seasons suitable for age dating was insufficient to allow for statistical comparisons of groundwater age between seasons; no single well was age dated during both seasons. It would be worthwhile to make such a comparison given the potential influence of residence time on geochemical reactions involving nitrate and selenium (Oremland and others, 1989; Bailey and others, 2014; Linard and others, 2016). Conceptually, it is possible that, in the irrigation season, the groundwater system is dominated by movement of relatively young water through fractures, shale partings, or bedding planes and that water levels subside in the nonirrigation season, in which case the groundwater system could contain a mixture of unflushed young water and older water that enters the fractures, shale partings, and (or) bedding planes from the shale matrix.

All age-dated samples plot on or very close to the model curves in figures 19 and 20, indicating those samples contained little, if any, water recharged prior to the early 1950 s (pre-1950s recharge). Samples that are a mixture of pre- and post-1950s recharge would plot below the model curves. Water from well 7 was not age dated, but its low tritium concentration indicates the water may have been mixed with some pre-1950s water (fig. 20). Water from well 7 had the maximum nitrate and selenate concentrations in the entire network (tables 8,9), which could point to another potential effect of residence time on nitrate and selenate concentrations. As 
Table 14. Estimates of groundwater age for select monitoring wells, east side of the Uncompahgre River, lower Gunnison River Basin, Colorado.

[ft, feet; ${ }^{\circ} \mathrm{C}$, degrees Celsius; \pm 1 sd, plus or minus 1 standard deviation; $\mathrm{cm}^{3} \mathrm{STP} / \mathrm{g}$, cubic centimeter at standard temperature and pressure per gram; $\mathrm{N}^{2}$, dinitrogen gas; mg/L, milligram per liter; Ne, neon; Ar, argon; Kr, krypton; Xe, xenon; CE, closed system equilibration; UA, unfractionated air; TU, tritium unit; $\mathrm{SF}_{6}$, sulfur hexafluoride; ${ }^{3} \mathrm{H}$, tritium; ${ }^{3} \mathrm{He}$ trit ${ }^{\text {tritogenic }}$ helium-3; pptv, parts per trillion by volume; DM, dispersion model; EMM, exponential mixing model; <, less than; --, not applicable. Altitude datum North American Vertical Datum 1988.]

\begin{tabular}{|c|c|c|c|c|c|c|c|c|c|c|c|c|c|c|c|c|c|}
\hline $\begin{array}{c}\text { Well } \\
\text { identifier }\end{array}$ & $\begin{array}{l}\text { Sample } \\
\text { date }\end{array}$ & $\begin{array}{l}\text { Recharge } \\
\text { altitiude } \\
\text { (ft) }\end{array}$ & $\begin{array}{c}\text { Recharge } \\
\text { temperature } \\
\left({ }^{\circ} \mathrm{C} \pm 1 \mathrm{sd}\right)\end{array}$ & $\begin{array}{c}\text { Excess } \\
\text { air } \\
\left(\mathrm{cm}^{3}\right. \\
\text { STP/g) }\end{array}$ & $\begin{array}{c}\text { Excess } \\
\mathrm{N}_{2} \\
(\mathrm{mg} / \mathrm{L})\end{array}$ & $\begin{array}{l}\text { Modeled } \\
\text { gases }\end{array}$ & $\begin{array}{l}\text { Excess } \\
\text { air } \\
\text { model }\end{array}$ & $\begin{array}{c}\text { Chi- } \\
\text { squared }\end{array}$ & $\begin{array}{l}\text { Fraction- } \\
\text { ation, } \\
\text { F }\end{array}$ & $\begin{array}{l}\text { Tritium } \\
\text { (TU } \\
\pm 1 \text { sd) }\end{array}$ & $\begin{array}{c}\mathrm{SF}_{6} \\
\text { (pptv } \pm 1 \\
\text { sd) }\end{array}$ & $\begin{array}{l}{ }^{3} \mathrm{He}_{\text {trit }} \\
\text { (TU } \\
\pm 1 \text { sd) }\end{array}$ & $\begin{array}{c}\text { Recharge } \\
\text { year }\end{array}$ & $\begin{array}{l}\text { Modeled } \\
\text { tracers }\end{array}$ & $\begin{array}{l}\text { Lumped- } \\
\text { parameter } \\
\text { model }\end{array}$ & $\begin{array}{l}\text { Dispersion } \\
\text { model } \\
\text { parameter }\end{array}$ & $\begin{array}{l}\text { Chi- } \\
\text { squared }\end{array}$ \\
\hline 2 & $3 / 5 / 2015$ & 5,285 & $13.7 \pm 1.9$ & 0.002 & $<1$ & $\mathrm{Ne}, \mathrm{Ar}, \mathrm{Kr}, \mathrm{Xe}$ & $\mathrm{CE}$ & 0.138 & 0.80 & $4.7 \pm 0.2$ & -- & $2.08 \pm 0.35$ & 2008 & ${ }^{3} \mathrm{H},{ }^{3} \mathrm{He}_{\text {trit }}$ & $\mathrm{DM}$ & 0.03 & 1.118 \\
\hline 5 & $8 / 6 / 2014$ & 5,161 & $14.1 \pm 1.5$ & 0.007 & $<1$ & $\mathrm{Ne}, \mathrm{Ar}, \mathrm{Kr}, \mathrm{Xe}$ & $\mathrm{CE}$ & 0.027 & 0.33 & -- & $5.1 \pm 1.3$ & $3.06 \pm 15$ & 2001 & $\mathrm{SF}_{6},{ }^{3} \mathrm{He}_{\text {trit }}$ & $\mathrm{DM}$ & 0.03 & 0.010 \\
\hline 8 & $3 / 5 / 2015$ & 5,302 & $9.3 \pm 1.6$ & 0.000 & 7 & $\mathrm{Kr}, \mathrm{Xe}$ & UA & -- & -- & $5.3 \pm 0.2$ & -- & $15.7 \pm 0.43$ & 2003 & ${ }^{3} \mathrm{H},{ }^{3} \mathrm{He}_{\text {trit }}$ & EMM & -- & 0.000 \\
\hline 10 & $3 / 11 / 2015$ & 5,482 & 10.6 & 0.008 & 0 & $\mathrm{Ar}, \mathrm{N}_{2}$ & UA & -- & -- & $4.4 \pm 0.2$ & $6.0 \pm 0.6$ & -- & 2005 & $\mathrm{SF}_{6},{ }^{3} \mathrm{H}$ & $\mathrm{DM}$ & 0.03 & 0.886 \\
\hline 17 & $3 / 6 / 2015$ & 5,226 & $11.6 \pm 2.2$ & 0.003 & 8 & $\mathrm{Ne}, \mathrm{Ar}, \mathrm{Kr}, \mathrm{Xe}$ & $\mathrm{CE}$ & 0.017 & 0.73 & $5.3 \pm 0.4$ & -- & $7.84 \pm 0.44$ & 2006 & ${ }^{3} \mathrm{H},{ }^{3} \mathrm{He}_{\text {trit }}$ & EMM & -- & 0.765 \\
\hline 18 & $3 / 10 / 2015$ & 5,059 & 10.6 & 0.003 & 0 & $\mathrm{Ar}, \mathrm{N}_{2}$ & UA & -- & -- & $5.7 \pm 0.3$ & $3.9 \pm 0.4$ & -- & 1995 & $\mathrm{SF}_{6},{ }^{3} \mathrm{H}$ & $\mathrm{DM}$ & 0.2 & 0.013 \\
\hline 25 & $3 / 3 / 2015$ & 6,119 & $9.0 \pm 0.6$ & 0.003 & 2 & $\mathrm{Ne}, \mathrm{Kr}, \mathrm{Xe}$ & UA & 0.007 & -- & $5.3 \pm 0.3$ & -- & $2.88 \pm 0.39$ & 2009 & ${ }^{3} \mathrm{H},{ }^{3} \mathrm{He}_{\text {trit }}$ & EMM & -- & 1.806 \\
\hline
\end{tabular}

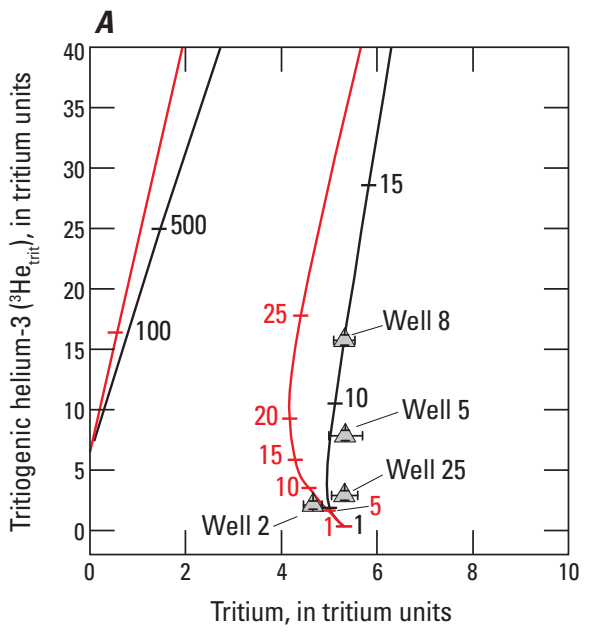

Tritium, in tritium units

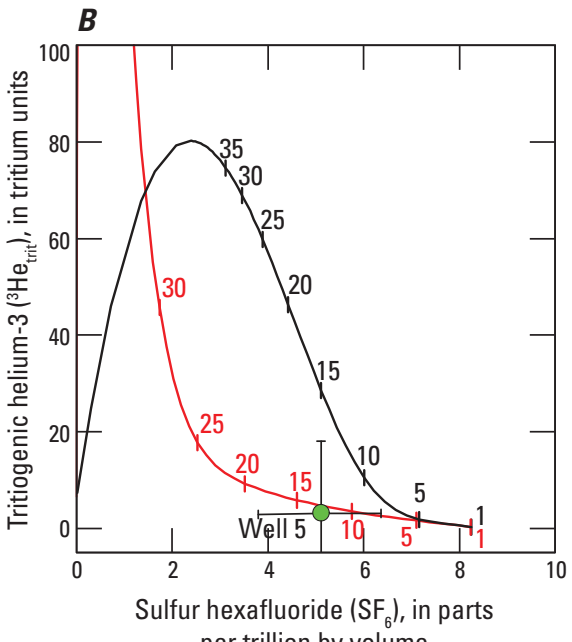

per trillion by volume

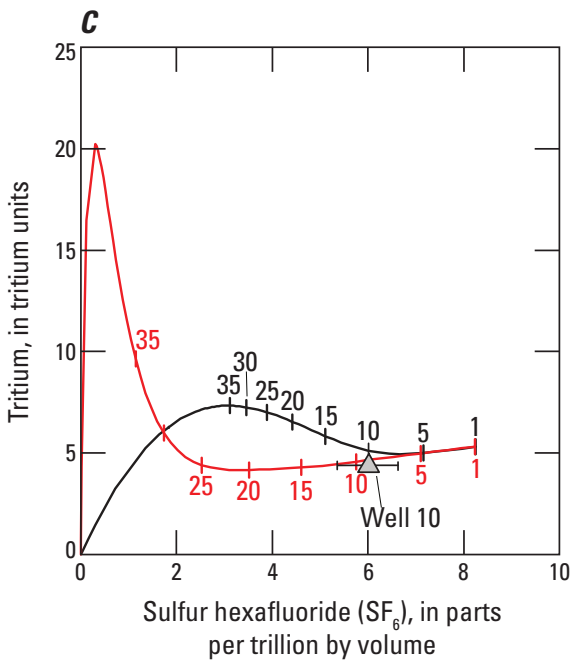

per trillion by volume

\title{
EXPLANATION
}

\author{
10
1 $\begin{gathered}\text { Dispersion model and mean age-Dispersion } \\ \text { parameter equals } 0.03\end{gathered}$ \\ X-and $y$-axis error bars-Error bar
represent \pm 1 standard deviation \\ Well 5 IIrigation season well and identifier \\ 10 Exponential-mixing model and mean age \\ Well ${ }^{10} \triangle$ Nonirrigation season well and identifier
}

Figure 19. Concentrations of tritium, ${ }^{3} \mathrm{He}_{\text {trit }}$ (tritiogenic helium-3), and $\mathrm{SF}_{6}$ (sulfur hexafluoride) in groundwater, 2014-15, and model results from TracerLPM (Jurgens and others, 2012). $A,{ }^{\text {trit }} \mathrm{He}_{\text {trit }}$ and tritium. $B,{ }^{3} \mathrm{He}_{\text {trit }}$ and $\mathrm{SF}_{6}$. $C$, Tritium and $\mathrm{SF}_{6}$. Error bars represent \pm 1 standard deviation. 


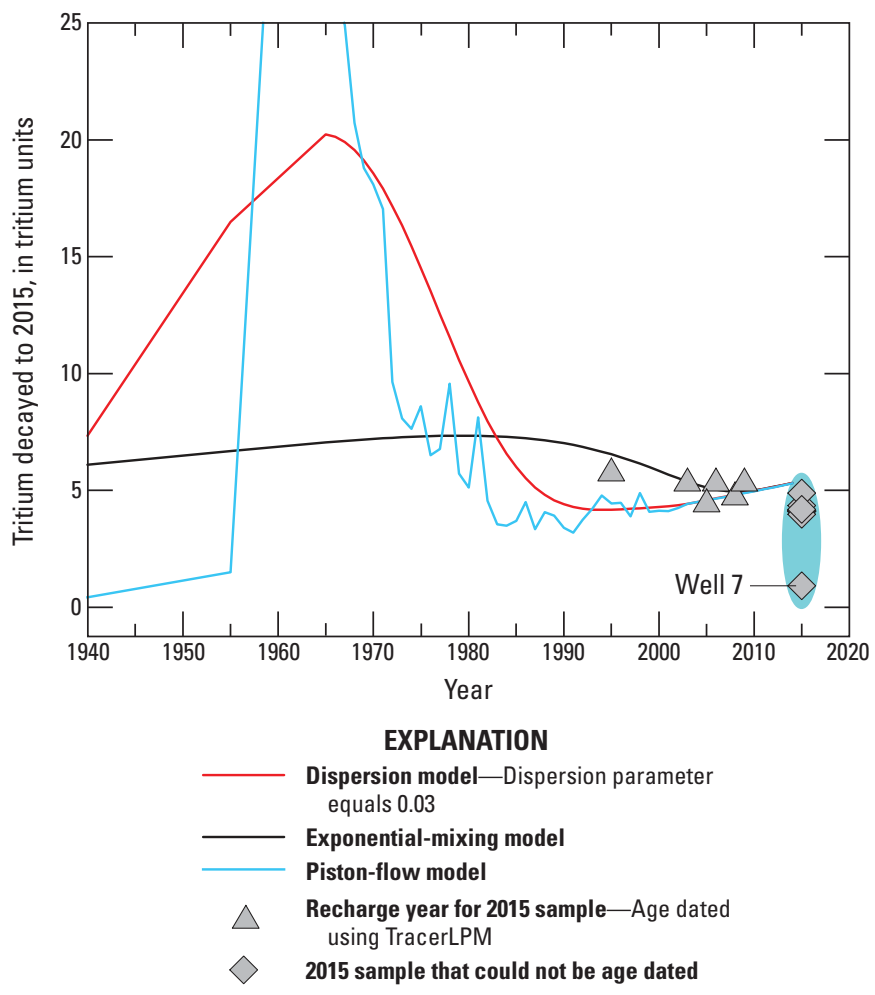

Figure 20. Tritium concentrations in samples collected in 2015 as a function of recharge year for the samples that were age dated or as a function of sample year for samples that were not age dated, and model results from TracerLPM (Jurgens and others, 2012).

previously discussed in the section "Redox Indicators," long residence times could potentially provide the time needed for redox processes to remove nitrate and selenate from solution by reducing them to less soluble forms. The data from well 7 suggest that long residence times, in some instances, might also provide longer reaction times for processes that release nitrate and selenate into solution. The results of a recent study (Linard and others, 2016) showing an increase in nitrate and selenate concentrations in groundwater with distance from a leaking canal appear to be consistent with this interpretation.

\section{Denitrification}

Multiple lines of evidence indicate that several samples were affected by denitrification (fig. 21). During the process of denitrification, nitrate is converted to $\mathrm{N}_{2}$ and the isotopic signature of both the reactant (nitrate) and the product $\left(\mathrm{N}_{2}\right)$ are changed according to variables such as reaction rates, concentrations of products and reactants, environmental conditions, and the species of the organism involved in the process (Cook and Herczeg, 2000). Generally speaking, the $\delta^{15} \mathrm{~N}_{-} \mathrm{NO}_{3}$ and $\delta^{18} \mathrm{O}-\mathrm{NO}_{3}$ of the remaining nitrate becomes isotopically heavier (more neutrons) (Cook and Herczeg, 2000). Nitrate isotope data collected during this study indicate that several samples were found to be isotopically heavy and defined trends in $\delta^{15} \mathrm{~N}-\mathrm{NO}_{3}$ with denitrification (fig. 21). Excess nitrogen was observed in several samples, again indicating denitrification because nitrogen gas is a product of nitrate reduction (fig. 18). Quantitative assessment of how much denitrification occurred could only be determined in the few samples that had dissolved-gas data unaffected by degassing. Excess nitrogen from denitrification ranged from $<1$ to $8 \mathrm{mg} / \mathrm{L}$ in those samples (table 14 ).

Small amounts of denitrification occurred in some wells with relatively low concentrations of both selenium and nitrate (wells 18, 24, and 25) (fig. 8, 21). However, based on $\mathrm{N}_{2}$ concentrations, there was not much nitrate in those samples before denitrification. In samples from wells such as 8 and 17 , which had relatively high concentrations of excess nitrogen (7-8 mg/L) (table 14), selenium concentrations remained relatively high (greater than $18.4 \mu \mathrm{g} / \mathrm{L}$ ) (fig. 8) because nitrate concentrations also remained relatively high (6.87-39.2 milligrams per liter as nitrogen) (fig. 21). In fact, the data on nitrate and excess nitrogen indicate that only 16 to 53 percent of the original nitrate in wells 8 and 17, respectively, was removed by denitrification. For samples that did not have data for $\delta^{18} \mathrm{O}-\mathrm{NO}_{3}$ or dissolved gases, some understanding of the extent of denitrification can still be obtained from the nitrate and $\delta^{15} \mathrm{~N}^{-\mathrm{NO}_{3}}$ data. Those data indicate that well 1 may have been highly denitrified relative to samples like those from wells 8 and 17 (fig. 21). The

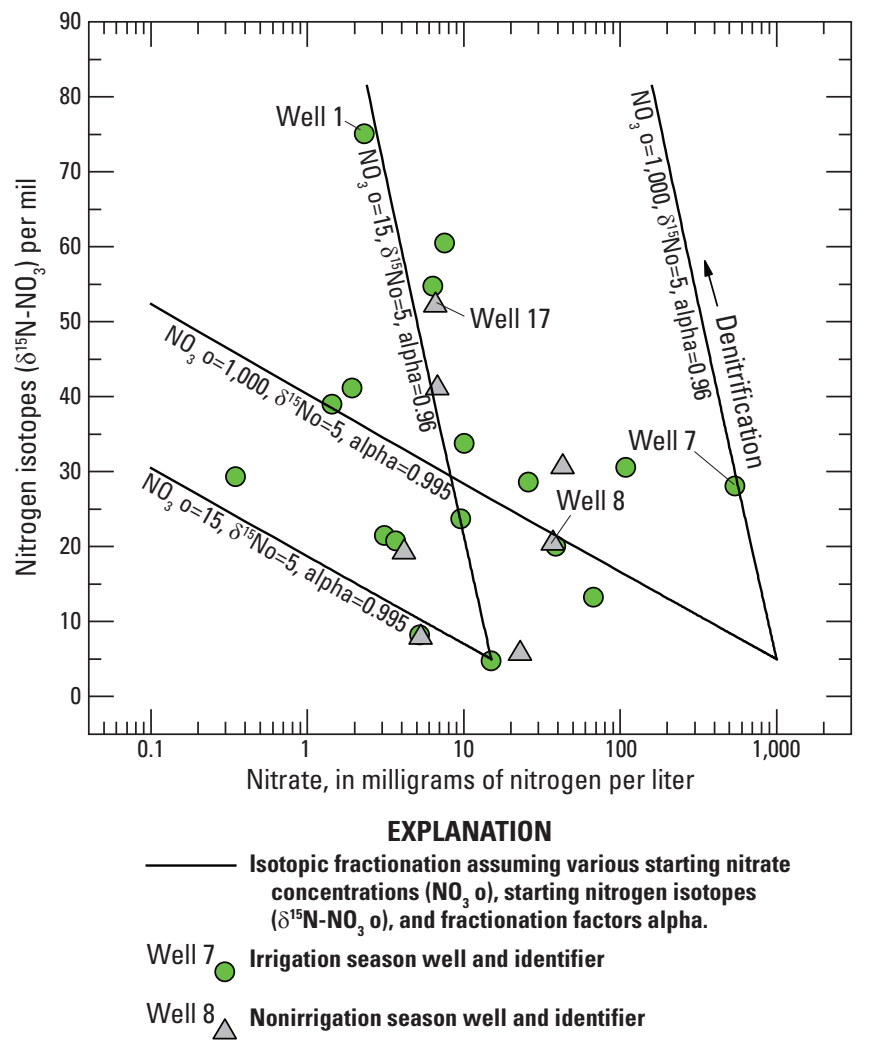

Figure 21. Isotopic fractionation of nitrate undergoing denitrification, assuming various starting nitrate concentrations and fractionation factors (alpha) (Cook and Herczeg, 2000). 
sample from well 1 may be an example of a sample that was sufficiently denitrified (nitrate of $<3$ milligrams per liter as nitrogen during the irrigation season) (fig. 21) such that selenium reduction could begin. In samples enriched with selenium and nitrate, denitrification is occurring but not at a rate sufficient to allow for selenium reduction.

\section{Estimates of Groundwater Discharge and Selenium Loading}

To approximate the amount of groundwater discharging to surface water, streamflow data were evaluated at Sunflower Drain (USGS ID 384551107591901) and Loutsenhizer Arroyo (USGS ID 383946107595301) for the period from 1991 to 2016 (USGS, 2017). A mean of instantaneous streamflows grouped by month were used to represent mean monthly values of the mean instantaneous values in cubic feet per second. Base flow was represented by a mean of streamflows measured in February when groundwater-level fluctuations exhibited low variability and contributions to streamflow from canals were absent (fig. 15). Mean monthly values were adjusted to represent cubic feet per month by first converting them to cubic feet per day and then multiplying by the number of days in the month. The mean instantaneous streamflow for February was converted to cubic feet per day and then multiplied by 365 days to represent base flow in cubic feet per year. Mean instantaneous streamflow in cubic feet per month were summed for all months to represent a total in cubic feet per year. At Sunflower Drain, base flow in cubic feet per year represented 10 percent of the total streamflow for the year (table 15), and at Loutsenhizer Arroyo, base flow in cubic feet per year represented 16 percent of the total streamflow for the year (table 16).

Groundwater discharges to surface water throughout the year in the study area and has been identified as an appreciable source of selenium load to surface water (Mast and others, 2014). Previous studies in Montrose Arroyo have estimated selenium load from groundwater at approximately 90 percent of the total selenium load (Butler, 2001). The annual selenium load at Sunflower Drain was 1,250 pounds per year, and base flow contributed 770 pounds per year, which represented 62 percent of the annual load (table 15). Applying the same approach at Loutsenhizer Arroyo, annual selenium load was 4,880 pounds per year, and base flow contributed 3,340 pounds per year, which represented 68 percent of the annual load (table 16). These results are consistent with base-flow estimates from a previous investigation that used a different approach-base flow represented by November, December, January, February, and March and provided a range of 46-59 percent of the annual load as base flow at Loutsenhizer Arroyo (Mast and others, 2014).

Table 15. Summary of selenium load at Sunflower Drain at Highway 92 (USGS ID 384551107591901) compared to estimated base-flow contribution, 1991-2016.

[February assumed to be most representative of base-flow contribution based on water-level data from the study area. $\mathrm{ft}^{3}$, cubic foot; $\mathrm{s}$, second; $\mathrm{d}$, day; mo, month; lb, pound; yr, year, \%, percent]

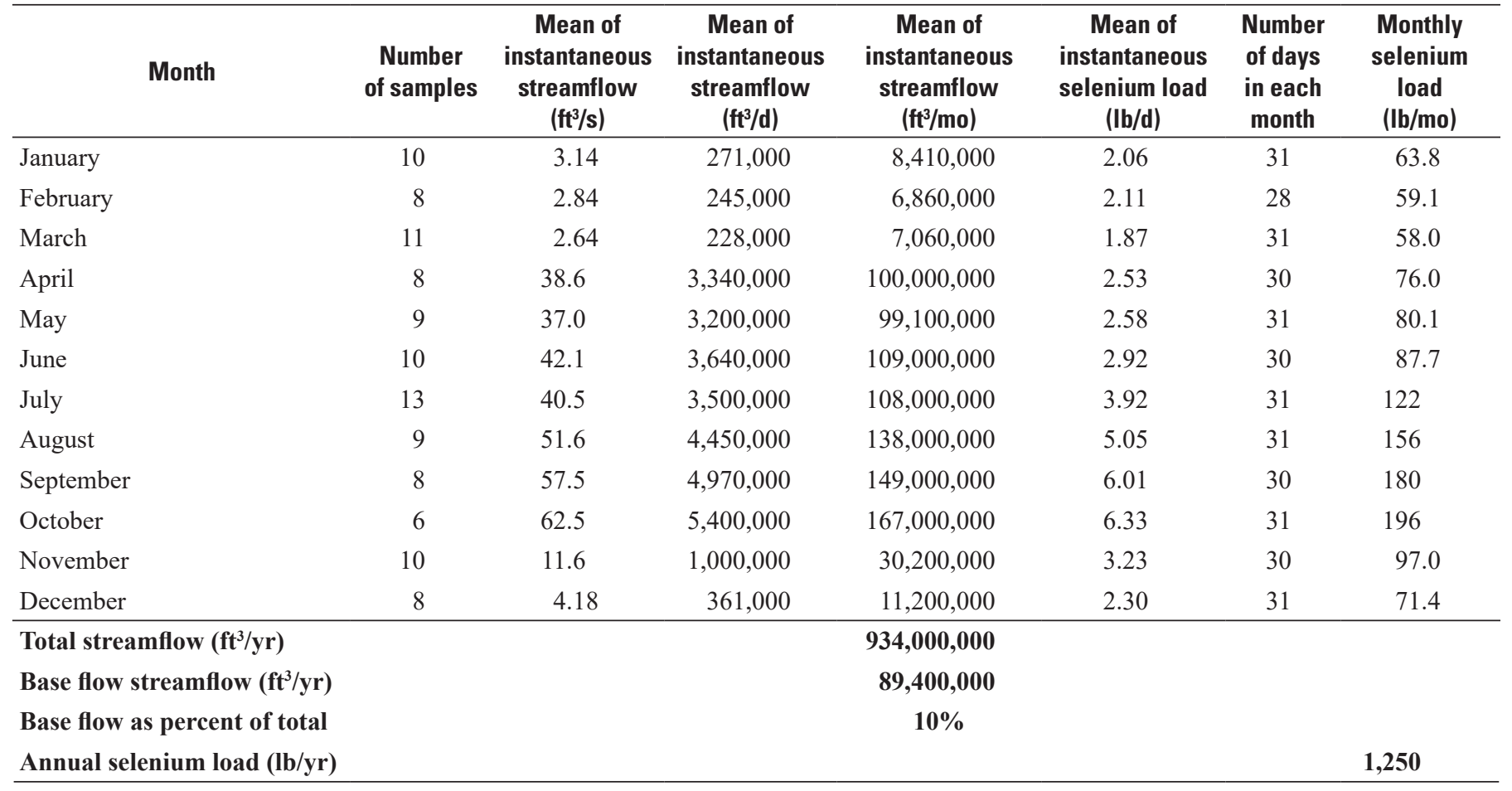


Table 16. Summary of selenium load at Loutsenhizer Arroyo below North River Road near Delta, Colo., (USGS ID 383946107595301) compared to estimated base-flow contribution, 1991-2016.

[February assumed to be most representative of base-flow contribution based on water-level data from the study area. $\mathrm{ft}^{3}$, cubic foot; s, second; $\mathrm{d}$, day; mo, month; lb, pound; yr, year, \%, percent]

\begin{tabular}{|c|c|c|c|c|c|c|c|}
\hline Month & $\begin{array}{l}\text { Number of } \\
\text { samples }\end{array}$ & $\begin{array}{c}\text { Mean of } \\
\text { instantaneous } \\
\text { streamflow } \\
\left(\mathrm{ft}^{3} / \mathrm{s}\right)\end{array}$ & $\begin{array}{c}\text { Mean of } \\
\text { instantaneous } \\
\text { streamflow } \\
\left(\mathrm{ft}^{3} / \mathrm{d}\right)\end{array}$ & $\begin{array}{c}\text { Mean of } \\
\text { instantaneous } \\
\text { streamflow } \\
\left(\mathrm{ft}^{3} / \mathbf{m o}\right)\end{array}$ & $\begin{array}{c}\text { Mean of } \\
\text { instantaneous } \\
\text { selenium load } \\
\text { (lb/d) }\end{array}$ & $\begin{array}{c}\text { Number } \\
\text { of days } \\
\text { in each } \\
\text { month }\end{array}$ & $\begin{array}{c}\text { Monthly } \\
\text { selenium } \\
\text { load } \\
(\mathrm{lb} / \mathrm{mo})\end{array}$ \\
\hline February & 10 & 9.24 & 798,000 & $22,400,000$ & 9.14 & 28 & 256 \\
\hline March & 17 & 13.3 & $1,150,000$ & $35,600,000$ & 11.9 & 31 & 369 \\
\hline May & 10 & 83.2 & $7,190,000$ & $223,000,000$ & 12.5 & 31 & 388 \\
\hline June & 11 & 96.8 & $8,370,000$ & $251,000,000$ & 12.3 & 30 & 369 \\
\hline July & 14 & 85.8 & $7,410,000$ & $230,000,000$ & 13.2 & 31 & 409 \\
\hline August & 13 & 102 & $8,810,000$ & $273,000,000$ & 16.4 & 31 & 508 \\
\hline September & 9 & 89.3 & $7,710,000$ & $231,000,000$ & 14.6 & 30 & 438 \\
\hline Total streamflow $\left(\mathrm{ft}^{3} / \mathbf{y r}\right)$ & & & & $1,790,000,000$ & & & \\
\hline Base flow streamflow $\left(\mathrm{ft}^{3} / \mathrm{yr}\right)$ & & & & $291,000,000$ & & & \\
\hline Base flow as percent of total & & & & $16 \%$ & & & \\
\hline Annual selenium load (lb/yr) & & & & & & & 4,880 \\
\hline
\end{tabular}

\section{Selenium Mobilization and Transport in Groundwater}

The installation of the 30 -well network allowed for the development of a conceptual model of selenium mobilization and transport in the shallow groundwater system on the east side of the Uncompahgre River (fig. 22). The conceptual model summarizes current understanding of selenium cycling in the shallow groundwater system by understanding vectors (recharge and flow paths), sources of selenium, geochemical reactions governing selenium mobilization and transport, and receptors (loading to streams).

The shallow groundwater system consists of a confined aquifer in the weathered Mancos Shale where water moves along bedding planes and in partings in the shale and an unconfined or semiconfined aquifer in the surficial deposits where water moves through unconsolidated material from areas of high potential to areas of lower potential. Water levels in both the confined and unconfined parts of the shallow groundwater system respond to the application of irrigation water, indicating a connection between surface activities and the shallow groundwater system.

Results of this study have provided an understanding of groundwater recharge and the general direction of groundwater flow. Based on findings presented in this report, the shallow groundwater system is primarily recharged by canal leakage and infiltration (deep percolation) of irrigation water (fig. 22). Groundwater flow in the study area followed the topography and generally flowed from south to north and from areas of high potential to areas of lower potential (for example, from canals to streams). The primary mechanism in the irrigation supply system on the east side of the Uncompahgre River is gravity; canals placed on hillsides (high potential) deliver water to fields (lower potential) (fig. 22). Sources of recharge to the shallow groundwater system indicated that the isotopic signature of groundwater was similar to that of local rivers and irrigation canals; there was no evidence of an older, isotopically different source of groundwater. Mean groundwater ages ranged from 6 to 20 years. Groundwater residence time could be an important control on selenium concentrations in groundwater systems similar to this one because selenate reduction by kinetically controlled microbial processes has the potential to remove selenium from solution given long enough residence times in the aquifer (Oremland and others, 1989; Böhlke and others, 2007; Bailey and others, 2014; Linard and others, 2016).

Results of this study have also improved understanding of recharge to the shallow groundwater system. Estimates of mean groundwater recharge to the unconfined aquifer range from 0.0 to $37.8 \mathrm{in} / \mathrm{yr}$. Estimates of groundwater recharge to the confined aquifer were beyond the scope of this current effort, but they would be important to understanding how 


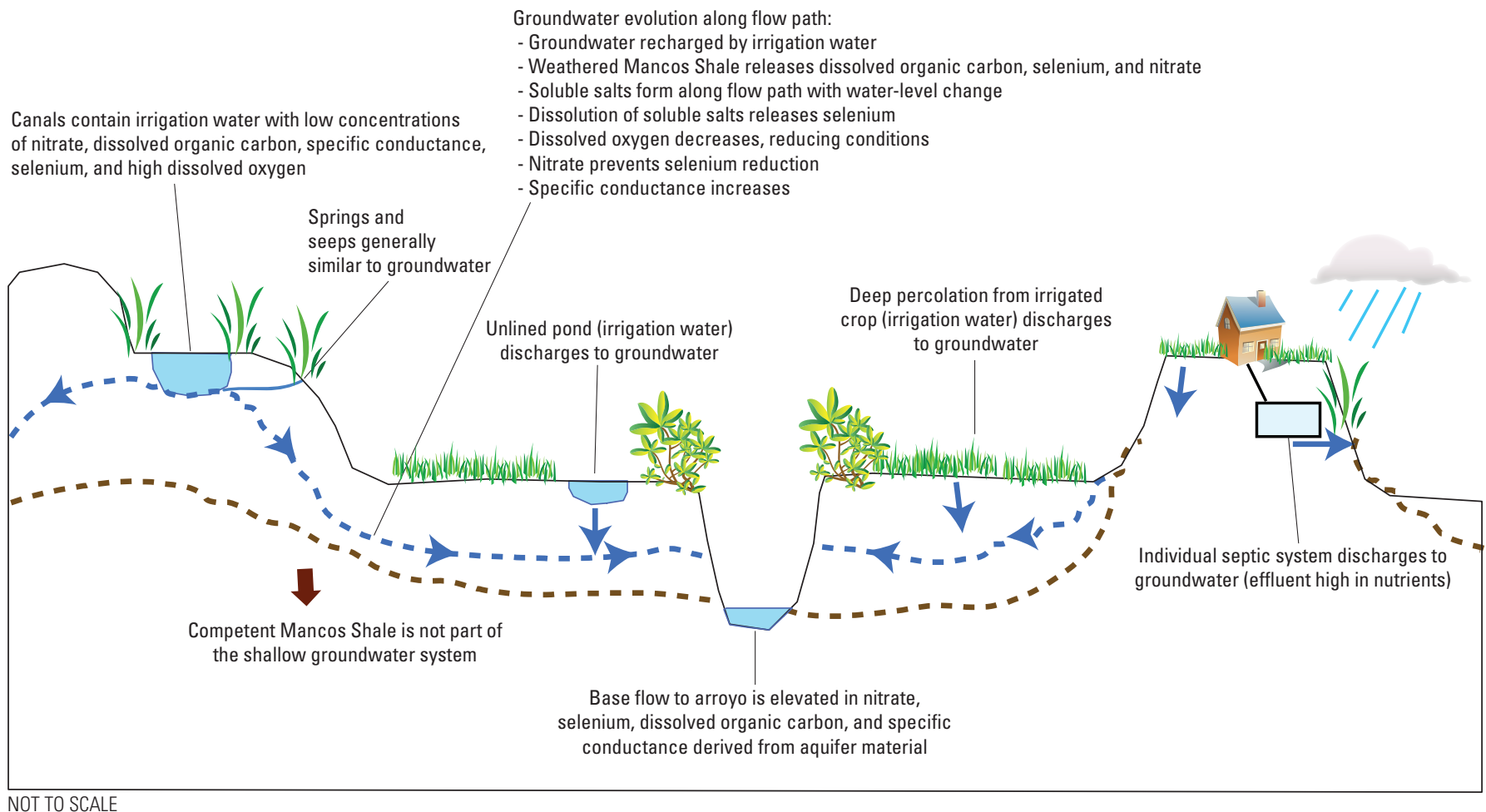

Figure 22. Conceptual model of selenium mobilization and transport in the shallow groundwater system on the east side of the Uncompahgre River, lower Gunnison River Basin, Colorado.

much recharge is entering the confined aquifer. Potential sources of recharge to the confined aquifer are canal leakage, where water enters the aquifer through bedding planes, and preferential flow paths like oblique fractures in the Mancos Shale. Other potential sources of recharge to the shallow groundwater system include unlined ponds and individual septic systems (fig. 22). The extent of these contributions is not well characterized, but these sources may become important remediation targets as agricultural sources of recharge decrease as the result of improvements in irrigation efficiency, canal piping, and changes in land use.

To provide some context for the potential contribution from septic systems, estimates of deep percolation were compared with septic contribution as recharge to the shallow groundwater system. Mean deep percolation for alfalfa crop sites was 1.27 acre-feet per irrigated acre, based on a study conducted in the Grand Valley (Mayo, 2008); multiplying percolation by the number of irrigated acres in the Gunnison Basin (177,404 acres) (Colorado Decision Support System, 2013) results in approximately 225,000 acre-feet per year of recharge to the shallow groundwater system. Water-use data from 2003 to 2008 provided by the Tri-County Water Conservancy District (2010) was used to estimate septic contribution. The mean water usage during January was assumed to represent the monthly amount of water that goes into septic and was estimated at 1,900 acre-feet per year (Tri-County Water Conservancy District, 2010). The volume of water estimated to contribute to groundwater from septic was appreciably less than that from deep percolation. Continued monitoring of the shallow groundwater system would provide information on how irrigation efficiencies affect water levels in the shallow groundwater system.

Groundwater-quality data from this study indicate that both weathering of Mancos Shale and dissolution of soluble salts were important mechanisms in the mobilization of selenium in the shallow groundwater system. The first step in selenium mobilization was the initial weathering of Mancos Shale some 20,000 years ago (Tuttle and others, 2014). This process formed gypsum and soluble organic matter, which are currently present in the weathered Mancos Shale (Tuttle and others, 2014). Data from this study indicate that selenium was highly correlated with nitrate and dissolved organic carbon, which in turn indicates that the Mancos Shale or its weathering products are the sources of these constituents. The Mancos Shale and its weathering products are broadly distributed across the study area and will continue to add high concentrations of dissolved nitrate, selenium, and dissolved organic carbon to groundwater through highly soluble salts and soluble organic material (Morrison and others, 2012; Mast and others, 2014; Linard and others, 2016; Mills and others, 2016). Once mobilized, selenium is stored in and released as highly soluble salts, primarily in surficial deposits (Mast and others, 2014; Mills and others, 2016). The formation of salts occurs during the wetting and drying of surficial deposits resulting from changes in water application during irrigation and nonirrigation seasons. Mast and others (2014) and Mills and others (2016) describe highly soluble salts as the primary source of 
selenium in the study area, making the distribution of these salts an important control on selenium mobilization.

Geochemical processes also act to control selenium mobility. The conditions in the shallow groundwater system are generally anoxic, which would typically reduce selenium from its mobile form to a less mobile form (for example, selenate to selenite); however, appreciable reduction of selenium was not observed in the groundwater-quality data. The primary reason for this lack of selenium reduction is the presence of elevated nitrate concentrations in groundwater, which inhibit selenium reduction (White and others, 1991). Denitrification is a mechanism for removing nitrate from groundwater, and though evidence of denitrification was observed, the rate of denitrification was not sufficient to reduce nitrate concentrations enough for selenium reduction from the groundwater.

Groundwater discharges to streams, arroyos, and drains throughout the study area, which substantially contributes to selenium loads. Although base flow only contributed 10 percent of the total streamflow at Sunflower Drain and 16 percent at Loutsenhizer Arroyo, it contributed 62 and 68 percent of the selenium load, respectively, at these sites. The remaining amount of selenium load at these sites not associated with base flow (38 and 32 percent, respectively) is not entirely composed of load produced by runoff (such as tail water or rainfall events) originating in the drainages. Both of these sites represent basins that receive recycled water containing selenium load that originated from upper portions of the system. The amount of selenium load entering Sunflower Drain during the irrigation season was approximately 6 pounds per day (selenium concentration data from USGS 384200107573901 East Canal Tail Water into Sunflower Drain [USGS, 2018]). Irrigation water applied to fields in Sunflower Drain contains selenium that originated from outside the drainage.

\section{Summary}

Dissolved selenium is a contaminant of concern in the lower Gunnison River Basin, Colorado. Selenium is naturally present in the Cretaceous Mancos Shale and is leached to groundwater and surface water by irrigation. On a regional scale, the Mancos Shale is not water bearing and is considered a confining unit because of its thickness and low permeability. On a local scale, wells have been completed in the weathered Mancos Shale, typically at shallow depths. The Colorado Department of Public Health and Environment has standards for dissolved selenium, and many rivers and streams in the lower Gunnison River Basin are designated as selenium impaired according to the chronic aquatic-life standard (4.6 micrograms per liter $[\mu \mathrm{g} / \mathrm{L}])$. Although little is known about groundwater's contribution to surface water, groundwater has often been implicated as a source of selenium to surface water.

In the environment, selenium has four oxidation states (-II, 0, IV, and VI), which can all occur together in soils. The most mobile form of selenium in the aqueous system is selenate (VI), but selenate can become immobile by reduction to elemental selenium (0), formation of metal selenides (-II), or selenite (IV) absorption. Reduction-oxidation (redox) processes are an important control on selenium in which the presence of higher redox species (oxygen and nitrate) prevents the reduction of mobile selenate to less mobile selenite.

The east side of the Uncompahgre River in Delta and Montrose Counties is one of the primary areas in the lower Gunnison River Basin where some of the highest selenium concentrations have been measured. Irrigation projects on the east side of the Uncompahgre River convey water that originates as snowmelt at higher elevation areas to lower elevation areas. Selenium concentrations measured during winter base flow indicate that shallow groundwater is enriched with respect to selenium. To characterize the hydrology and groundwater quality of the shallow groundwater system, the U.S. Geological Survey, in cooperation with the Bureau of Reclamation, the Colorado Water Conservation Board, and the Gunnison Basin Selenium Management Program, established a 30-well groundwatermonitoring network on irrigated land on the east side of the Uncompahgre River in the lower Gunnison River Basin.

Monitoring wells in the network were sampled on multiple occasions to assess groundwater quality, sources of recharge, and groundwater age in the shallow groundwater system. The first 10 wells were sampled in August 2013, and all 30 wells were sampled in August 2014 and again in March 2015 to determine field properties and to determine the concentration of major ions, trace elements, selenium speciation (selenite and selenite), nutrients, dissolved organic carbon, hydrogen and oxygen isotopes in water (deuterium-protium ratio $\left[\delta^{2} \mathrm{H}-\mathrm{H}_{2} \mathrm{O}\right]$ and delta oxygen- $\left.18\left[\delta^{18} \mathrm{O}-\mathrm{H}_{2} \mathrm{O}\right]\right)$ referred to as water isotopes, stable nitrogen and oxygen isotopes in nitrate (delta Nitrogen-15 in nitrate fraction $\left[\delta^{15} \mathrm{~N}^{-\mathrm{NO}_{3}}\right]$ and delta Oxygen-18 in nitrate fraction $\left[\delta^{18} \mathrm{O}-\mathrm{NO}_{3}\right]$ ) referred to as nitrate isotopes, tritium, noble gases, sulfur hexafluoride $\left(\mathrm{SF}_{6}\right)$, and dissolved gases,. The 30 -well network provided a spatial distribution to understand groundwater quality over the study area. Wells were completed in both surficial deposits and the weathered Mancos Shale to evaluate the role that geologic material has with respect to groundwater quality.

Sampling during both the irrigation and nonirrigation seasons provides some understanding of seasonality and irrigation effects. No temporal pattern in specific conductance values of groundwater was observed between the irrigation season and the nonirrigation season; however, the specific conductance of applied irrigation water was considerably less than that of groundwater. Specific conductance values in groundwater ranged from 1,500 to 44,000 microsiemens per centimeter at 25 degrees Celsius $\left(\mu \mathrm{S} / \mathrm{cm}\right.$ at $\left.25^{\circ} \mathrm{C}\right)$, with a median value of $4,200 \mu \mathrm{S} / \mathrm{cm}$. In comparison, specific conductance of surface water at the Uncompahgre River at Colona, Colo., was $500 \mu \mathrm{S} / \mathrm{cm}$ (annual statistic for water year 2011). Because irrigation water is thought to be the primary source of recharge to the groundwater system, it would seem possible that specific conductance of groundwater would decrease during the irrigation season with the influx of fresh irrigation water to 
the system; however, no decrease was observed. One interpretation of this observation is that the monitoring wells were relatively far from the sources of recharge and that water from those wells was in contact with aquifer materials for a long time relative to newly recharged water. In addition to water residence times near aquifer materials, the existence and distribution of soluble salts in the aquifer itself are an important source of dissolved solids throughout the study area.

Groundwater types in the study area were calcium sulfate, mixed-cation sulfate, and sodium sulfate. Through use of specific conductance values, major-ion data were grouped into 25th, 50th, and 75th percentiles. Specific conductance values in the 75th percentile and greater were found in association with sodium-sulfate-type waters, and specific conductance values in the 25 th percentile were found in association with calcium-sulfate-type waters.

Nitrate and dissolved organic carbon were found in high concentrations in wells in the study area. Concentrations of dissolved nitrate plus nitrite in groundwater ranged from below the limit of detection to 550 milligrams per liter as nitrogen, with a median concentration of 4.8 milligrams per liter as nitrogen. Dissolved organic carbon concentrations ranged from 1.82 to 82 milligrams per liter $(\mathrm{mg} / \mathrm{L})$, with a median concentration of $6.8 \mathrm{mg} / \mathrm{L}$. A high degree of spatial variability was observed in measurements of both nitrate and dissolved organic carbon concentrations. Nitrate concentrations measured in this study are comparable to concentrations measured in groundwater and pore water in areas of the Mancos Shale unaffected by irrigated agriculture, indicating that the Mancos Shale is the primary source of both of these constituents in the shallow groundwater system.

Prior to this study, little was known about the concentrations of selenium in the shallow groundwater system, even though groundwater was believed to be an appreciable source of selenium to surface water. Although high selenium concentrations were observed in samples collected from wells, selenium concentrations were found to be highly variable across the well network. Concentrations of dissolved selenium ranged from below the limit of detection to $4,100 \mu \mathrm{g} / \mathrm{L}$, with a median concentration of $14 \mu \mathrm{g} / \mathrm{L}$. Selenium was present mostly in its oxidized form, selenate. Selenite concentrations were often below detection or much less than selenate concentrations. No temporal or spatial pattern emerged with regard to selenium concentration. Selenium concentrations were not significantly different between the irrigation season and the nonirrigation season $(p=0.202)$, despite the influx of irrigation water, which generally has selenium concentrations much less than that observed in groundwater. Selenium concentrations in surficial deposits (median of $23 \mu \mathrm{g} / \mathrm{L}$ ) were not significantly greater than selenium concentrations in the weathered Mancos Shale (median of $5 \mu \mathrm{g} / \mathrm{L}, \mathrm{p}=0.0755$ ).

Understanding redox processes is important to understanding selenium speciation and mobility. Selenate, the most mobile form of selenium, is generally mobile under oxygenand nitrate-reducing conditions. However, once nitrate concentrations are reduced to low levels (less than $5 \mathrm{mg} / \mathrm{L}$ ) under nitrate-reducing conditions, selenate can be reduced to less mobile forms. Previous studies have found that nitrate concentrations of 5 milligrams per liter as nitrogen or greater inhibit the reduction of selenate. The conditions in the shallow groundwater system are generally anoxic, however, several redox processes were identified in the shallow groundwater system and sorted into 3 groups according to their redox category: group 1 was oxic and mixed (oxic-anoxic), group 2 was mixed (anoxic), and group 3 was anoxic. Selenate concentrations in groups 1 and 2 were not significantly different from one another $(p=0.061)$, whereas concentrations in group 3 were significantly lower than concentrations in groups 1 and $2(p<0.01)$. This pattern is consistent with results from previous studies, showing that highly reducing conditions maintain selenate concentrations at low levels.

Multiple lines of evidence indicate that groundwater was affected by denitrification. The amount of denitrification that had occurred, however, could only be determined in a few samples. Small amounts of denitrification occurred in some wells with relatively low concentrations of both selenium and nitrate (wells 18, 24, and 25), However, based on nitrogen-gas concentrations, there was not much nitrate in those samples before denitrification. In samples from wells 8 and 17, which had relatively high concentrations of excess nitrogen $(7-8 \mathrm{mg} / \mathrm{L})$, selenate concentrations remained high $(89.9-558 \mu \mathrm{g} / \mathrm{L})$ because nitrate concentrations also remained high $(6.87-39.2 \mathrm{mg} / \mathrm{L})$.

Selenium has been identified as the primary constituent of concern in surface water in the lower Gunnison River Basin, but groundwater samples show high concentrations of other trace elements, most notably boron, iron, manganese, lithium, strontium, and uranium. Several trace element concentrations in groundwater samples were found to exceed Colorado groundwater agricultural standards; the samples indicate that groundwater enriches surface water in these trace elements.

By using data collected as part of this study, the shallow groundwater system can be characterized according to hydraulic conductivity, water level and potentiometric surface, estimates of groundwater recharge, sources of recharge and groundwater ages, and estimates of groundwater discharge and selenium loading from groundwater. The shallow groundwater system would not likely have contained appreciable quantities of water prior to the development of irrigation in the study area, but irrigation now provides substantial quantities of water, allowing for the existence of a shallow groundwater system. The shallow groundwater system is generally unconfined but is confined or semiconfined where clay sediments predominate or where wells were completed in the weathered Mancos Shale. Wells screened in surficial deposits when confined conditions were observed in the aquifer at the time of the slug test are considered to be semiconfined. Of the wells used in this study, 12 were completed in the weathered Mancos Shale and 18 were completed in surficial deposits, which include alluvial gravel, sand, silt, and clay deposited in major river valleys and tributary drainages, in alluvial fans, and as older alluvial terrace, valley fill, or fan deposits. The 12 wells completed in the 
weathered Mancos Shale had water-bearing zones in partings in the shale that were typically less than 5 feet thick.

Hydraulic-conductivity values for surficial deposits ranged from 0.06 to 100 feet per day $(\mathrm{ft} / \mathrm{d})$, with a median of $3.5 \mathrm{ft} / \mathrm{d}$, geometric mean of $3.0 \mathrm{ft} / \mathrm{d}$, and standard deviation of $26 \mathrm{ft} / \mathrm{d}$ (18 wells). Hydraulic-conductivity values for weathered Mancos Shale ranged from 0.3 to $50 \mathrm{ft} / \mathrm{d}$, with a median of $6.0 \mathrm{ft} / \mathrm{d}$, geometric mean of $4.6 \mathrm{ft} / \mathrm{d}$, and standard deviation of $16 \mathrm{ft} / \mathrm{d}$ (12 wells).

Depth to water below land surface, as represented by the potentiometric surface, generally was less than 20 feet throughout the study area. Where consolidated bedrock occurs at or near the land surface, the altitude of the potentiometric surface might be less than the altitude of the bedrock surface, indicating that the shallow groundwater system in these areas may be nonexistent or thin, discontinuous, and transient. The direction of groundwater flow in the shallow groundwater system generally was from topographically high areas toward the stream valleys of the Gunnison and Uncompahgre Rivers, Loutsenhizer Arroyo, and large tributary drainages. A groundwater divide located along the topographic divide between the Uncompahgre River and Loutsenhizer Arroyo marks where the direction of groundwater flow changes between the two stream valleys. All wells indicated that water levels respond to the application of irrigation water; response was similar for wells screened in either surficial deposits or the weathered Mancos Shale. Although irrigation water is typically used from early April to late October, minimum and maximum depths to water varied temporally by well location. The variability in the timing of minimum depths to water between wells may indicate differences in rates of recharge or distances of wells from recharge sources.

Recharge to the shallow groundwater system occurs primarily during the irrigation season as water fills the canals and is applied to fields, with little recharge from precipitation. The amount of recharge to the unconfined portion of the shallow groundwater system was estimated by using the graphical approach to the water-table fluctuation method, in which the antecedent recession curves were extrapolated manually to obtain the peak water-table rise. Estimated recharge values range from 0.0 to 37.8 inches per year (in/yr) and a mean of $9.4 \mathrm{in} / \mathrm{yr}$. The stable isotopic composition of groundwater was used to help determine sources of recharge to the shallow groundwater system and to determine whether processes like evaporation affected recharge. Isotopic data are consistent with water diverted from local rivers and reservoirs being the primary source of recharge to the shallow groundwater system. Assuming relatively little temporal variability in the isotopic composition of deep groundwater, the data indicate deep groundwater was not an important source of recharge to the shallow groundwater system.

Seven samples had tracer data suitable for age dating. Mean groundwater ages in the samples based on these models range from 6 to 20 years and correspond to recharge years ranging from 1995 to 2009. The number of samples from the irrigation and nonirrigation seasons suitable for age dating was insufficient to allow for statistical comparisons of groundwater age between seasons; no single well was age dated during both seasons. Such a comparison could be worthwhile given the potential influence of residence time on geochemical reactions involving nitrate and selenium. Conceptually, it is possible that, in the irrigation season, the groundwater system is dominated by movement of relatively young water through fractures, shale partings, or bedding planes and that water levels subside in the nonirrigation season, in which case the groundwater system could contain a mixture of unflushed young water and older water that enters the fractures, shale partings, and (or) bedding planes from the shale matrix.

To approximate the amount of groundwater discharging to surface water, streamflow data were evaluated at Sunflower Drain and Loutsenhizer Arroyo for the period from 1991 to 2016. Base flow was represented by a mean of streamflows measured in February when water-level fluctuations exhibited low variability and contributions to streamflow from canals were absent. Base flow at Sunflower Drain represented 10 percent of the total streamflow, in cubic feet per year, and base flow at Loutsenhizer Arroyo represented 16 percent of the total streamflow, in cubic feet per year. Although base flow contributed a small percentage of the total streamflow, it played an appreciably greater role in selenium load. The annual selenium load contributed from base flow at Sunflower Drain was estimated at 770 pounds, (62 percent of annual load), and at Loutsenhizer Arroyo, 3,340 pounds, (68 percent of annual load). These results are consistent with base-flow estimates from previous investigations, which ranged from 46 to 59 percent of the annual load as base flow at Loutsenhizer Arroyo.

The installation of the 30-well network allowed for the development of a conceptual model of selenium mobilization and transport in the shallow groundwater system on the east side of the Uncompahgre River. The conceptual model summarizes current understanding of selenium cycling in the shallow groundwater system by understanding vectors (recharge and flow paths), sources of selenium, geochemical reactions governing selenium mobilization and transport, and receptors (loading to streams). Results of this study have provided an understanding of groundwater recharge and the general direction of groundwater flow; recharge occurs primarily from canal leakage and deep percolation of irrigation water. The primary mechanism in the irrigation supply system in the study area is gravity; canals placed on hillsides (high potential) deliver water to fields (lower potential). In addition to irrigation, other potential sources of recharge to the shallow groundwater system include unlined ponds and individual septic systems. The extent of these contributions is not well characterized, but these sources may become important remediation targets as agricultural sources of recharge decrease as the result of improvements in irrigation efficiency, canal piping, and changes in land use.

The first step in selenium mobilization was the initial weathering of Mancos Shale some 20,000 years ago. This process formed gypsum and soluble organic matter, which are currently present in the weathered Mancos Shale. The Mancos Shale and its weathering products are broadly distributed across the study area 
and will continue to add high concentrations of dissolved nitrate, selenium, and dissolved organic carbon to groundwater through highly soluble salts and soluble organic material.

Geochemical processes also act to control selenium mobility. The conditions in the shallow groundwater system are generally anoxic, which would typically reduce selenium from its mobile form to a less mobile form (for example, selenate to selenite); however, appreciable reduction of selenium was not observed in the groundwater-quality data. The primary reason for this lack of selenium reduction is the presence of elevated nitrate concentrations in groundwater, which inhibit selenium reduction. Although there is evidence of denitrification in the shallow groundwater system, the rate of denitrification was not sufficient to reduce nitrate concentrations enough for selenium reduction.

Base flow contributed 62 and 68 percent of the selenium load, respectively, at Sunflower Drain and Loutsenhizer Arroyo. The remaining amount of selenium load at these sites is likely not the sole product of runoff (as tail water or rainfall events) originating in the drainages. Both of these sites represent basins that receive recycled water containing selenium load that originated from upper portions of the system. In Sunflower Drain, the amount of selenium load entering the drainage system during the irrigation season was estimated at approximately 6 pounds per day. Irrigation water applied to fields in Sunflower Drain contain selenium that originated from outside the drainage.

\section{References Cited}

Aeschbach-Hertig, W., Peeters, F., Beyerle, U., and Kipfer, R., 1999, Interpretation of dissolved atmospheric noble gases in natural waters: Water Resources Research, v. 35, p. 2779-2792.

Arnold, L.R., 2017, Geospatial datasets of regolith thickness, bedrock altitude, depth to water, potentiometric-surface altitude, and saturated thickness for the shallow groundwater system in the lower Gunnison River Basin, Delta, Montrose, Ouray, and Gunnison Counties, Colorado: U.S. Geological Survey data release, accessed Fall 2017, at https://doi.org/10.5066/F70863S6.

Bailey, R.T., Gates, T.K., and Ahmadi, M., 2014, Simulating reactive transport of selenium coupled with nitrogen in a regional-scale irrigated groundwater system: Journal of Hydrology, v. 515, p. 29-46.

Bailey, R.T., Hunter, W.J., and Gates, T.K., 2012, The influence of nitrate on selenium in irrigated agricultural groundwater systems: Journal of Environmental Quality, v. 41, p. 783-792.

Böhlke, J.K., and Denver, J.M., 1995, Combined use of groundwater dating, chemical, and isotopic analyses to resolve the history and fate of nitrate contamination in two agricultural watersheds, Atlantic coastal plain, Maryland: Water Resources Research, v. 31, p. 2319-2339.
Böhlke, J.K., Verstraeten, I.M., and Kraemer, T.F., 2007, Effects of surface-water irrigation on sources, fluxes, and residence times of water, nitrate, and uranium in an alluvial aquifer: Applied Geochemistry, v. 22, p. 152-174.

Bouwer, H., and Rice, R.C., 1976, A slug test method for determining hydraulic conductivity of unconfined aquifers with completely or partially penetrating wells: Water Resources Research, v. 12, no. 3, p. 423-428.

Brenton, R.W., and Arnett, T.L., 1993, Methods of analysis by the U.S. Geological Survey National Water Quality Laboratory-Determination of dissolved organic carbon by UV-promoted persulfate oxidation and infrared spectrometry: U.S. Geological Survey Open-File Report 92-480, 12 p.

Briggs, P.H., and Crock, J.G., 1986, Automated determination of total selenium in rocks, soils, and plants: U.S. Geological Survey Open-File Report 86-40, 20 p.

Brooks, T., and Ackerman, D.J., 1985, Reconnaissance of ground-water resources in the lower Gunnison River Basin, southwestern Colorado: U.S. Geological Survey WaterResources Investigations Report 84-4185, 30 p., 2 pls.

Bureau of Reclamation [Reclamation], 1982, Hydrosalinity, appendix B of Colorado River water quality improvement program-Lower Gunnison Basin unit—Feasibility report: Bureau of Reclamation, $86 \mathrm{p}$.

Bureau of Reclamation [Reclamation], 2011, Selenium management program-Program formulation document, Gunnison River Basin, Colorado: Bureau of Reclamation, prepared by Selenium Management Program Workgroup, $80 \mathrm{p}$.

Bureau of Reclamation [Reclamation], 2017, Uncompahgre project: Bureau of Reclamation web page, accessed May 25, 2017, at https://www.usbr.gov/projects/index.php?id=412.

Busenberg, E., and Plummer, N.L., 2000, Dating young groundwater with sulfur hexafluoride-Natural and anthropogenic sources of sulfur hexafluoride: Water Resources Research, v. 36, p. 3011-3030.

Butler, D.L., 2001, Effects of piping irrigation laterals on selenium and salt loads, Montrose Arroyo Basin, Western Colorado: U.S. Geological Survey Water-Resources Investigations Report 01-4204, 14 p.

Butler, D.L., Krueger, R.P., Osmundson, B.C., Thompson, A.L., and McCall, S.K., 1991, Reconnaissance investigation of water quality, bottom sediment, and biota associated with irrigation drainage in the Gunnison and Uncompahgre River Basins and at Sweitzer Lake, west-central Colorado, 1988-89: U.S. Geological Survey Water-Resources Investigations Report 91-4103, 99 p. 
Butler, D.L., and Leib, K.J., 2002, Characterization of selenium in the lower Gunnison River Basin, Colorado, 1988-2000: U.S. Geological Survey Water-Resources Investigations Report 02-4151, 26 p.

Butler, D.L., Wright, W.G., Stewart, K.C., Osmundson, B.C., Krueger, R.P., and Crabtree, D.W., 1996, Detailed study of selenium and other constituents in water, bottom sediment, soil, alfalfa, and biota associated with irrigation drainage in the Uncompahgre Project area and in the Grand Valley, west-central Colorado, 1991-93: U.S. Geological Survey Water-Resources Investigations Report 96-4138, 136 p.

Butler, J.J., Jr., Garnett, E.J., and Healey, J.M., 2003, Analysis of slug tests in formations of high hydraulic conductivity: Ground Water, v. 41, no. 5, p. 620-630.

Casciotti, K.L., Sigman, D.M., Galanter Hastings, M., Böhlke, J.K., and Hilkert, A., 2002, Measurement of the oxygen isotopic composition of nitrate in seawater and freshwater using the denitrifier method: Analytical Chemistry, v. 74, p. $4905-4912$.

Cleveland, W.S., 1981, LOWESS: A program for smoothing scatterplots by robust locally weighted regression. The American Statistician, 35, 54. https://doi.org/10.2307/2683591.

Colorado Decision Support System, 2013, GIS dataDivision 4, Gunnison [downloadable data files]: Colorado Decision Support System web page, accessed September 11, 2013, at http://cdss.state.co.us/GIS/Pages/ Division4Gunnison.aspx.

Colorado Department of Public Health and Environment [CDPHE], 2016, Water quality standards Surface water quality classifications and standards (currently effective): Regulation 31, [downloadable data files]: Colorado Department of Public Health and Environment web page, accessed October 11, 2016, at https://www.colorado.gov/pacific/ cdphe/water-quality-control-commission-regulations.

Colorado Division of Water Resources, 2012a, Well-application information: Colorado Division of Water Resources, accessed May 1, 2012, at http://cdss.state.co.us/GIS/Pages/ AllGISData.aspx.

Colorado Division of Water Resources, 2012b, Wellpermit information [database]: Colorado Division of Water Resources, accessed September 24, 2012, at http://dwrweblink.state.co.us/dwrweblink/.

Cook, P.G., and Herczeg, A.L., 2000, Environmental tracers in subsurface hydrology: Kluwer Academic Publishers, p. 261-297.

Coplen, T.B., Böhlke, J.K., and Casciotti, K.L., 2004, Using dual-bacterial denitrification to improve $\delta^{15} \mathrm{~N}$ determinations of nitrates containing mass-independent ${ }^{17} \mathrm{O}$ : Rapid Communications in Mass Spectrometry, v. 18, p. 245-250.
Coplen, T.B., Herczeg, A.L., and Barnes, C., 2000, Isotope engineering-Using stable isotopes of the water molecule to solve practical problems, in Cook, P.G., and Herczeg, A.L., eds., Environmental tracers in subsurface hydrology: Boston, Kluwer Academic Publishers, p. 79-110.

Coplen, T.B., and Kendall, C., 2000, Stable isotope and oxygen isotope ratios for selected sites of the U.S. Geological Survey's NASQAN and Benchmark surface-water networks: U.S. Geological Survey Open-File Report 00-160, 409 p.

Coplen, T.B., Qi, H., Révész, K., Casciotti, K., and Hannon, J.E., 2012, Determination of the $\delta^{15} \mathrm{~N}$ and $\delta^{18} \mathrm{O}$ of nitrate in water-RSIL lab code 2900 (ver. 1.1, September 2012): U.S. Geological Survey Techniques and Methods, book 10, chap. C17, 35 p., accessed Fall 2017, at https://pubs.usgs.gov/tm/2006/tm10c17/. [Supersedes version 1.0, released in 2007.]

Cunningham, W.L., and Schalk, C.W., comps., 2011, Groundwater technical procedures of the U.S. Geological Survey: U.S. Geological Survey Techniques and Methods, book 1, chap. A1, 151 p., accessed Fall 2017, at https://pubs.usgs.gov/tm/1a1/.

Delin, G.N., Healy, R.W., Lorenz, D.L., and Nimmo, J.R., 2007, Comparison of local- to regional-scale estimates of ground-water recharge in Minnesota, USA: Journal of Hydrology, v. 334, no. 1-2, p. 231-249.

Esri, Inc., 1999-2015, ArcGIS 10.3.1 for desktop: Redlands, Calif., Esri, Inc., accessed Fall 2017, at http://www.esri.com/.

Farnsworth, R.K., and Thompson, E.S., 1982, Mean monthly, seasonal, and annual pan evaporation for the United States: National Oceanic and Atmospheric Administration Technical Report NWS 34, 91 p.

Fishman, M.J., 1993, Methods of analysis by the U.S. Geological Survey National Water Quality LaboratoryDetermination of inorganic and organic constituents in water and fluvial sediments: U.S. Geological Survey Open-File Report 93-125, $217 \mathrm{p}$.

Fishman, M.J., and Friedman, L.C., eds., 1989, Methods for determination of inorganic substances in water and fluvial sediments: U.S. Geological Survey Techniques of WaterResources Investigations, book 5, chap. A1, 545 p.

Freeman, L.A., Carpenter, M.C., Rosenberry, D.O., Rousseau, J.P., Unger, R. and McLean, J.C. 2004, Use of submersible pressure transducers in water-resources investigations: U.S. Geological Survey Techniques of Water-Resources Investigations, book 8, chap. A3, 52 p., accessed January 1, 2013 at https://doi.org/10.3133/twri08A3.

Garbarino, J.R., Kanagy, L.K., and Cree, M.E., 2006, Determination of elements in natural-water, biota, sediment and soil samples using collision/reaction cell inductively coupled plasma-mass spectrometry: U.S. Geological Survey Techniques and Methods, book 5, chap. B1, 88 p. 
Greene, E.A., and Shapiro, A.M., 1998, AIRSLUG-A Fortran program for the computation of type curves to estimate transmissivity and storativity from prematurely terminated air-pressurized slug tests: Ground Water, v. 36, no. 2, p. 373-376.

Gunnison Basin Selenium Task Force, 2017, Current projects-Effects of changes in land use on selenium loading in the Whitewater, CO area: Gunnison Basin Selenium Task Force web page, accessed May 25, 2017, at http://www.seleniumtaskforce.org/whitewaterco.html.

Halford, K.J., and Kuniansky, E.L., 2002, Documentation of spreadsheets for the analysis of aquifer-test and slug-test data: U.S. Geological Survey Open-File Report 02-197, 54 p.

Heaton, T.H.E., and Vogel, J.C., 1981, "Excess air" in groundwater: Journal of Hydrology, v. 50, p. 201-216.

Helsel, D.R., and Hirsch, R.M., 2002, Statistical methods in water resources: U.S. Geological Survey Techniques of Water-Resources Investigations, book 4, chap. A3, 534 p.

Hem, J.D., 1985, Study and interpretation of the chemical characteristics of natural water: U.S. Geological Survey Water-Supply Paper 2254, 264 p.

Holloway, J.M., and Smith, R.L., 2005, Nitrogen and carbon flow from rock to water-Regulation through soil biogeochemical processes, Mokelumne River watershed, California and Grand Valley, Colorado: Journal of Geophysical Research-Earth Surface Processes, v. 110, article F01010, 12 p., accessed Fall 2017, at https://doi.org/10.1029/2004JF000124.

Hunt, A.G., 2015, Noble Gas Laboratory's standard operating procedures for the measurement of dissolved gas in water samples: U.S. Geological Survey Techniques and Methods, book 5, chap. A11, 22 p., accessed Fall 2017, at https://doi.org/10.3133/tm5A11.

Izett, G.A., Wilcox, R.E., Obradovich, J.D., and Reynolds, R.L., 1971, Evidence for two Pearlette-like ash beds in Nebraska and adjoining areas [abs.]: Geological Society of America Abstracts with Programs, v. 3, no. 4, p. 265-266.

Johnson, A.I., 1967, Specific yield-Compilation of specific yields for various materials: U.S. Geological Survey WaterSupply Paper 1662-D, 74 p.

Jurgens, B.C., Böhlke, J.K., and Eberts, S.M., 2012, TracerLPM (version 1)-An Excel ${ }^{\mathbb{Q}}$ workbook for interpreting groundwater age distributions from environmental tracer data: U.S. Geological Survey Techniques and Methods, book 4, chap. F3, 60 p.

Jurgens, B.C., Böhlke, J.K., Kauffman, L.J., Belitz, K., and Esser, B.K., 2016, A partial exponential lumped parameter model to evaluate groundwater age distributions and nitrate trends in long-screened wells: Journal of Hydrology, v. 543, p. 109-126.
Langmuir, D., 1997, Aqueous environmental geochemistry: Upper Saddle River, N.J., Prentice Hall, Inc., 600 p.

Linard, J.I., 2013, Ranking contributing areas of salt and selenium in the lower Gunnison River Basin, Colorado, using multiple linear regression models: U.S. Geological Survey Scientific Investigations Report 2013-5075, 35 p., accessed Fall 2017, at http://pubs.usgs.gov/sir/2013/5075/.

Linard, J.I., McMahon, P.B., Arnold, L.R., Thomas, J.C., 2016, Response of selenium concentrations in groundwater to seasonal canal leakage, lower Gunnison River Basin, Colorado, 2013: U.S. Geological Survey Scientific Investigations Report 2016-5047, 30 p., accessed Fall 2017, at https://doi.org/10.3133/sir20165047.

Lohman, S.W., 1965, Geology and artesian water supply, Grand Junction area, Colorado: U.S. Geological Survey Professional Paper 451, 149 p.

Lohman, S.W., 1972, Ground-water hydraulics: U.S. Geological Survey Professional Paper 708, 70 p., 9 pls.

Mamyrin, B.A., and Tolstikhin, I.N., 1984, Helium isotopes in nature: New York, Elsevier, 273 p.

Mast, M.A., Mills, T.J., Paschke, S.S., Keith, G., and Linard, J.I., 2014, Mobilization of selenium from the Mancos Shale and associated soils in the lower Uncompahgre River Basin, Colorado: Applied Geochemistry, v. 48, p. 16-27.

Mayo, J.W., 2008, Estimating the effects of conversion of agricultural land to urban land on deep percolation of irrigation water in the Grand Valley, western Colorado: U.S. Geological Survey Scientific Investigations Report 2008-5086, 58 p.

McGookey, D.P., Haun, J.D., Hale, L.A., Goodell, H.A., McCubbin, D.G., Weimer, R.J., Wulf, G.R., and Cobban, W.A., 1972, Cretaceous system, in Mallory, W.W., Nolte, C.J., Jensen, F.S., and Griffith, E.G., eds., Geologic atlas of the Rocky Mountain region: Denver, Colo., Rocky Mountain Association of Geologists, p. 190-228.

McMahon, P.B., and Chapelle, F.H., 2008, Redox processes and water quality of selected principal aquifers: Ground Water, v. 46, no. 2, p. 259-271.

McNeal, J.M., and Balistrieri, L.S., 1989, Geochemistry and occurrence of selenium-An overview, in Jacobs, L.W., ed., Selenium in agriculture and the environment: Soil Science Society of America, Special Publication 23, p. 1-13.

Michel, R.L., 1989, Tritium deposition in the continental United States, 1953-83: U.S. Geological Survey WaterResources Investigations Report 89-4072, 46 p. 
Mills, T.J., Mast, M.A., Thomas, J.C., and Keith, G., 2016, Controls on selenium distribution and mobilization in an irrigated shallow groundwater system underlain by Mancos Shale, Uncompahgre River Basin, Colorado, USA: Science of the Total Environment, v. 566-567, p. 1621-1631.

Moore, J.L., 2011, Characterization of salinity and selenium loading and land-use change in Montrose Arroyo, western, Colorado, from 1992 to 2010: U.S. Geological Survey Scientific Investigations Report 2011-5106, 23 p.

Morrison, S.J., Goodknight, C.S., Tigar, A.D., Bush, R.P, and Gil, A., 2012, Naturally occurring contamination in the Mancos Shale: Environmental Science and Technology, v. 46, p. 1379-1387.

Mueller, D.K., Schertz, T.L., Martin, J.D., and Sandstrom, M.W., 2015, Design, analysis, and interpretation of field quality-control data for water-sampling projects: U.S. Geological Survey Techniques and Methods, book 4, chap. C4, 54 p., accessed Fall 2017, at https://doi.org/10.3133/tm4C4.

Noe, D.C., Morgan, M.L., Hanson, P.R., and Keller, S.M., 2007, Geologic map of the Montrose East quadrangle, Montrose County, Colorado: Colorado Geological Survey Open-File Report OF-07-02, scale 1:24,000.

Oremland, R.S., Hollibaugh, J.T., Maest, A.S., Presser, T.S., Miller, L.G., and Culbertson, C.W., 1989, Selenate reduction to elemental selenium by anaerobic bacteria in sediments and culture-Biogeochemical significance of a novel sulfate-independent respiration: Applied and Environmental Microbiology, v. 55, p. 2333-2343.

Paschke, S.S., Runkel, R.L., Walton-Day, K., Kimball, B.A., and Schaffrath, K.R., 2012, Streamflow and water-quality conditions including selenium sources and processes affecting selenium loading in the Toll Gate Creek watershed, Aurora, Arapahoe County, Colorado, 2007: U.S. Geological Survey Scientific Investigations Report 2012-5280, 108 p.

Paschke, S.S., Walton-Day, K., Beck, J.A., Webber, A., and Dupree, J.A., 2014, Geologic sources and concentrations of selenium in the west-central Denver Basin, including the Toll Gate Creek watershed, Aurora, Colorado, 2003-2007: U.S. Geological Survey Scientific Investigations Report 2013-5099, 30 p., accessed Fall 2017, at https://doi.org/10.3133/sir20135099.

Putnam, J.E., and Hansen, C.V., 2014, Quality-assurance and data management plan for groundwater activities by the U.S. Geological Survey in Kansas, 2014: U.S. Geological Survey Open-File Report 2014-1060, 37 p., accessed Fall 2017, at https://doi.org/10.3133/ofr20141060.

Révész, K., and Coplen, T.B., 2006, Methods of the Reston Stable Isotope Laboratory: U.S. Geological Survey Techniques and Methods, book 10, chap. C1, 27 p.
Richards, R.J., and Moore, J.L., 2015, Characterization of streamflow, salinity, and selenium loading and land-use change in Montrose Arroyo, western Colorado, from 1992 to 2013: U.S. Geological Survey Scientific Investigations Report 2015-5039, 18 p., accessed Fall 2017, at https://doi.org/10.3133/sir20155039.

Scott, J.C., 1990, Computerized stratified random siteselection approaches for design of a ground-water-quality sampling network: U.S. Geological Survey Water-Resources Investigations Report 90-4101, 109 p.

Sigman, D.M., Casciotti, K.L., Andreani, M., Barford, C., Galanter, M., and Böhlke, J.K., 2001, A bacterial method for the nitrogen isotopic analysis of nitrate in seawater and freshwater: Analytical Chemistry, v. 73, p. 4145-4153.

Solomon, D.K., and Cook, P.G., 2000, ${ }^{3} \mathrm{H}$ and ${ }^{3} \mathrm{He}$, in Cook, P.G., and Herczeg, A.L., eds., Environmental tracers in subsurface hydrology: Boston, Kluwer Academic Publishers, p. 197-424.

Thatcher, L.L., 1962, The distribution of tritium fallout in precipitation over North America: International Association of Scientific Hydrology Bulletin, v. 7, p. 48-58.

Thomas, J.C., 2015, Installation of a groundwater monitoringwell network on the east side of the Uncompahgre River in the lower Gunnison River Basin, Colorado, 2014: U.S. Geological Survey Data Series 955, 44 p. [Also available at https://doi.org/10.3133/ds955.]

Thomas, J.C., and Arnold, L.R., 2015, Installation of a groundwater monitoring-well network on the east side of the Uncompahgre River in the lower Gunnison River Basin, Colorado, 2012: U.S. Geological Survey Data Series 923, 29 p. [Also available at https://doi.org/10.3133/ds923.]

Tri-County Water Conservancy District, 2010, Tri-County Water Conservancy District water conservation plan: Montrose, Colo., Tri-County Water Conservancy District, accessed April 10, 2018, at http://www.tricountywater.org/ www/pdf/info/000029/wcp.pdf.

Tuttle, M.L.W., Fahy, J.W., Elliott, J.G., Grauch, R.I., and Stillings, L.L., 2014, Contaminants from Cretaceous black shale-I. Natural weathering processes controlling contaminant cycling in Mancos Shale, southwestern United States, with emphasis on salinity and selenium: Applied Geochemistry, v. 46, p. 57-71, accessed Fall 2017, at https://pubs.er.usgs.gov/publication/70056935.

Tweto, O., 1979, Geologic map of Colorado: U.S. Geological Survey, 2 sheets, scale 1:500,000.

U.S. Environmental Protection Agency [EPA], 2017, National primary drinking-water standards: U.S. Environmental Protection Agency Office of Water fact sheet EPA 816-F-03-016, accessed December 28, 2017, at http://www.epa.gov/safewater/contaminants/index.html. 
U.S. Geological Survey [USGS], 2013, The National Map-1 arc-second National Elevation Dataset: U.S. Geological Survey raster digital data, accessed October 2, 2015, at http://viewer.nationalmap.gov/basic.

U.S. Geological Survey [USGS], 2015a, Reston Stable Isotope Laboratory (RSIL): U.S. Geological Survey web page, accessed August 1, 2015, at https://isotopes.usgs.gov/.

U.S. Geological Survey [USGS], 2015b, The Reston Groundwater Dating Laboratory U.S. Geological Survey web page, accessed August 1, 2015, at https://water.usgs.gov/lab/.

U.S. Geological Survey [USGS], 2017, National Water Information System: U.S. Geological Survey database, accessed September 28, 2017, at https://doi.org/10.5066/F7P55KJN.

\section{U.S. Geological Survey [USGS], 2018, USGS} 384200107573901 EAST CANAL TAILWATER INTO SUNFLOWER DRAIN, accessed October 22, 2018 at https://waterdata.usgs.gov/nwis/inventory?agency_ code=USGS\&site_no=384200107573901.

U.S. Geological Survey [USGS], variously dated, National field manual for the collection of water-quality data: U.S. Geological Survey Techniques of Water-Resources Investigations, book 9, chaps. A1-A10, accessed Fall 2017, at http://pubs.water.usgs.gov/twri9A.

Warner, J.W., Heimes, F.J, and Middelburg, R.F., 1985, Ground-water contribution to the salinity of the Upper Colorado River Basin: U.S. Geological Survey WaterResources Investigations Report 84-4198, 113 p.
Western Regional Climate Center, 2017, Cooperative climatological data summaries: Western Regional Climate Center database, accessed December 6, 2017, at https://wrcc.dri.edu/summary/Climsmco.html.

White, A.F., Benson, S.M., Yee, A.W., Wollenberg, H.A., Jr., and Flexser, S., 1991, Groundwater contamination at the Kesterson Reservoir, California-2. Geochemical parameters influencing selenium mobility: Water Resources Research, v. 27, p. 1085-1098.

Williams, T, Foreman, W, 2015, Changes to National Water Quality Laboratory (NWQL) procedures used to establish and verify laboratory detection and reporting limits, accessed March 28, 2019, at https://nwql.usgs.gov/Public/ tech_memos/nwq1.2015-02.pdf.
Publishing support provided by the Science Publishing Network, Denver Publishing Service Center

For more information concerning the research in this report, contact the

Director, USGS Colorado Water Science Center

Box 25046, Mail Stop 415

Denver, CO 80225

(303) 236-4882

Or visit the Colorado Water Science Center website at https://co.water.usgs.gov. 


\section{Appendix 1. Estimates of Recharge Using the Graphical Approach to the Water Table Fluctuation (WTF) Method}

The amount of recharge to the unconfined portion of the shallow groundwater system was estimated by using the graphical approach to the water table fluctuation (WTF) method, in which the antecedent recession curves were extrapolated manually to obtain the peak water-table rise (Delin and others, 2007). Specific yield (Sy) values were estimated from knowledge of the lithologic material for each well and compared to values derived by Johnson (1967). Each figure contains time series plots of depth to water with antecedent recession curves shown with dashed lines (figures 1.1-1.14). Each figure also contains peak water-table rise value(s) in red. For figures 1.2, 1.10, and 1.14 , time series plots include both manual and continuous depths to water.

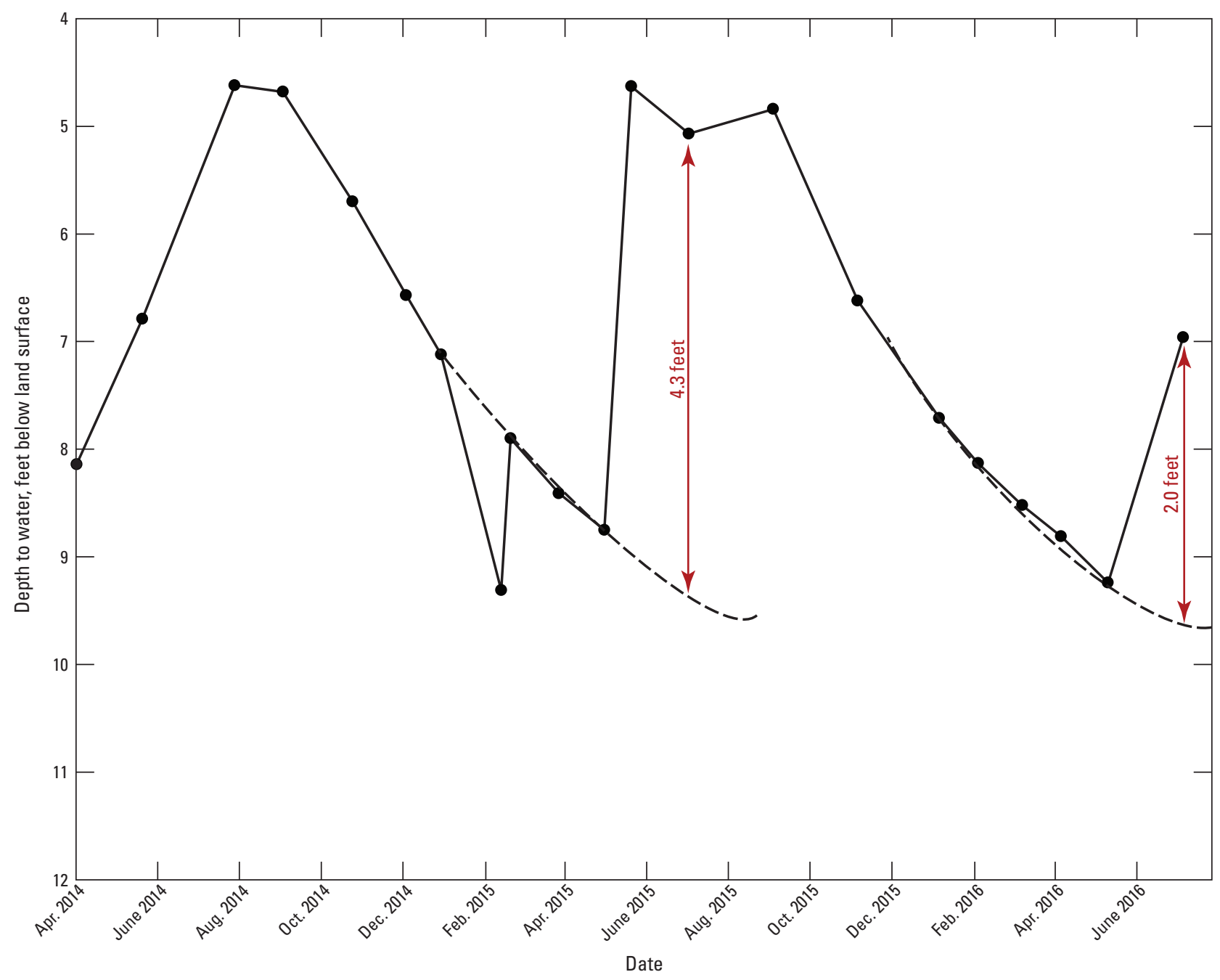

Figure 1.1. Peak water-table rise values (in red) used to determine estimated recharge for well 2 based on the graphical approach to the water-table fluctuation method. 


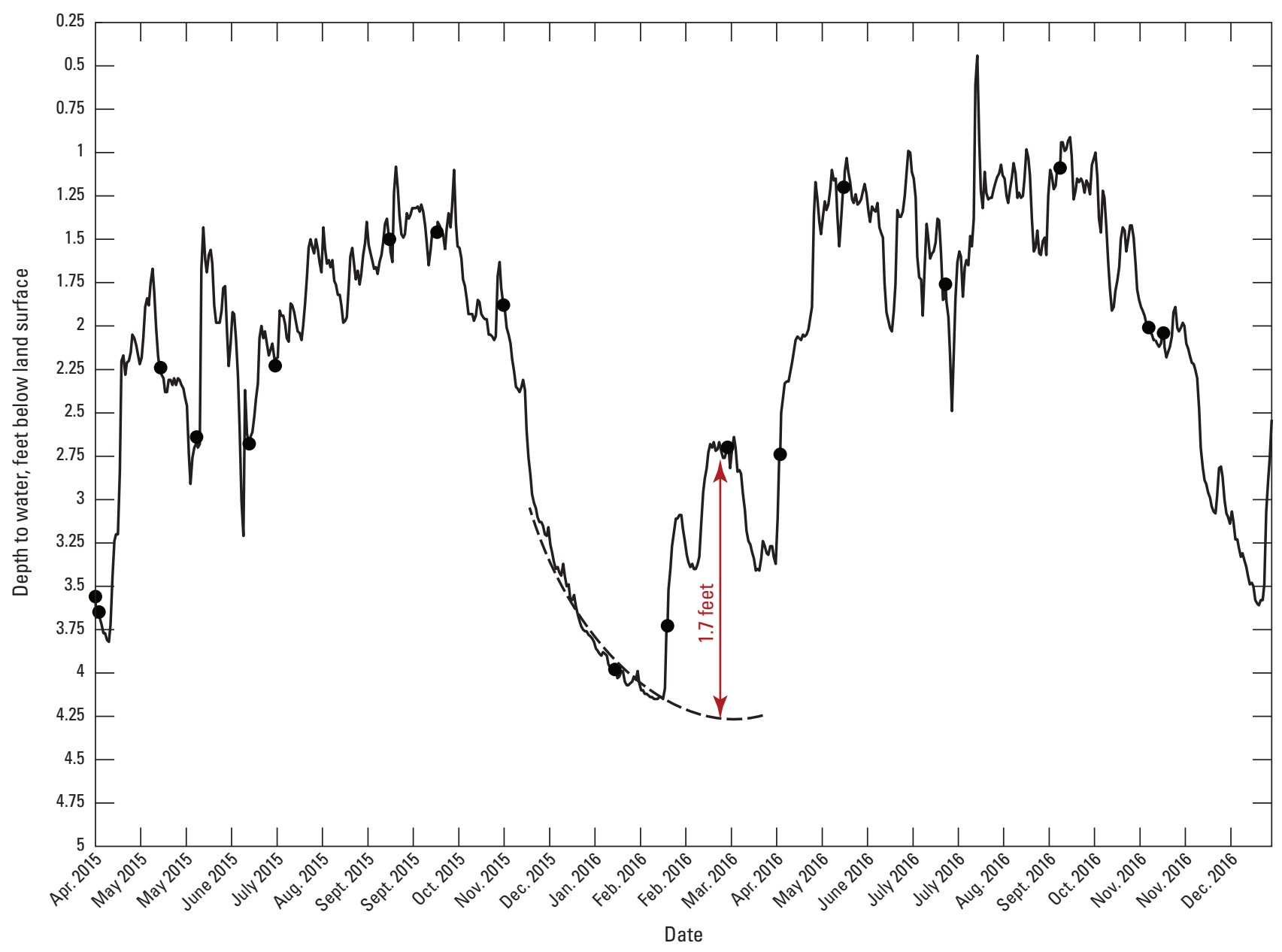

Figure 1.2. Peak water-table rise values (in red) used to determine estimated recharge for well 3 based on the graphical approach to the water-table fluctuation method. 


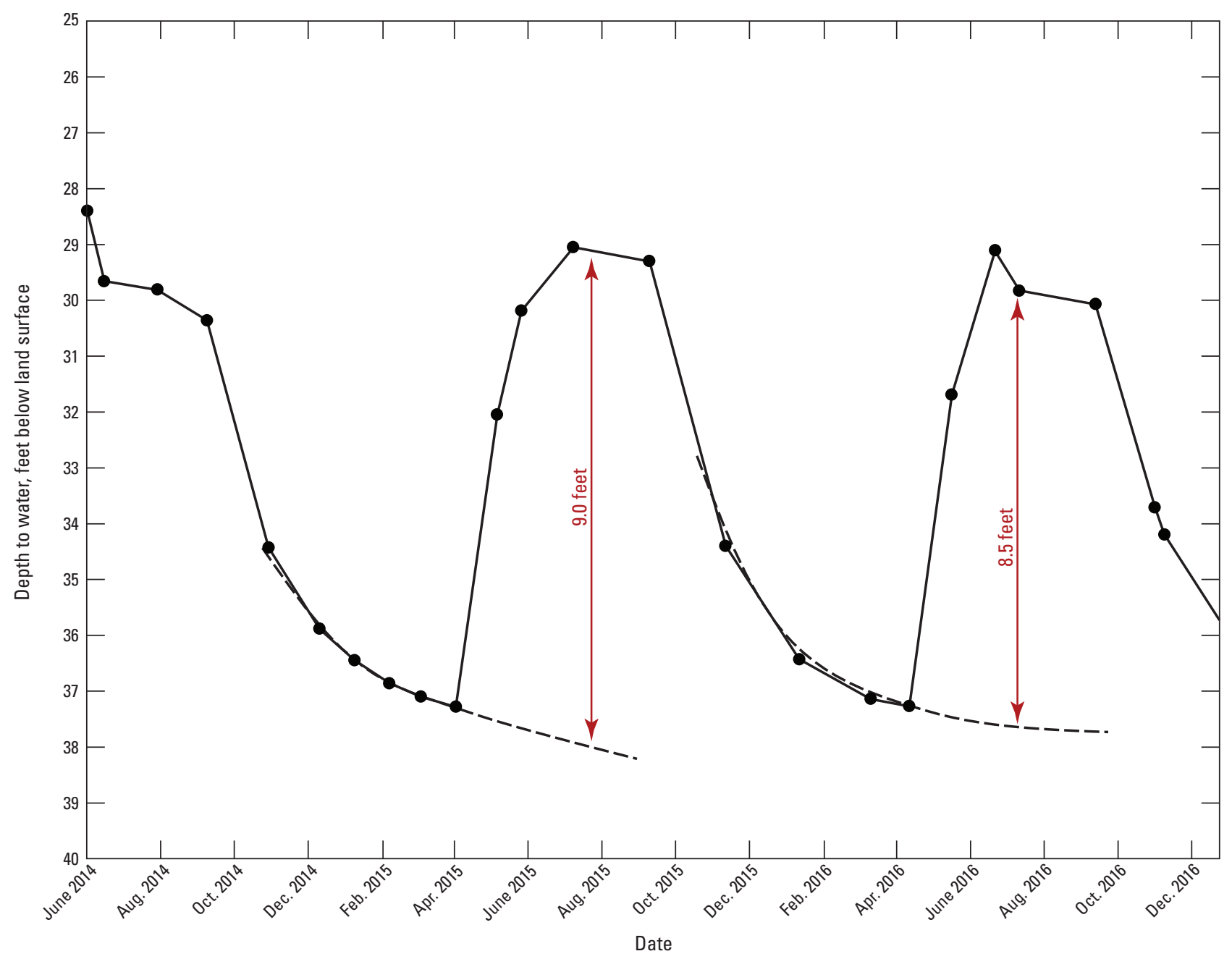

Figure 1.3. Peak water-table rise values (in red) used to determine estimated recharge for well 6 based on the graphical approach to the water-table fluctuation method. 


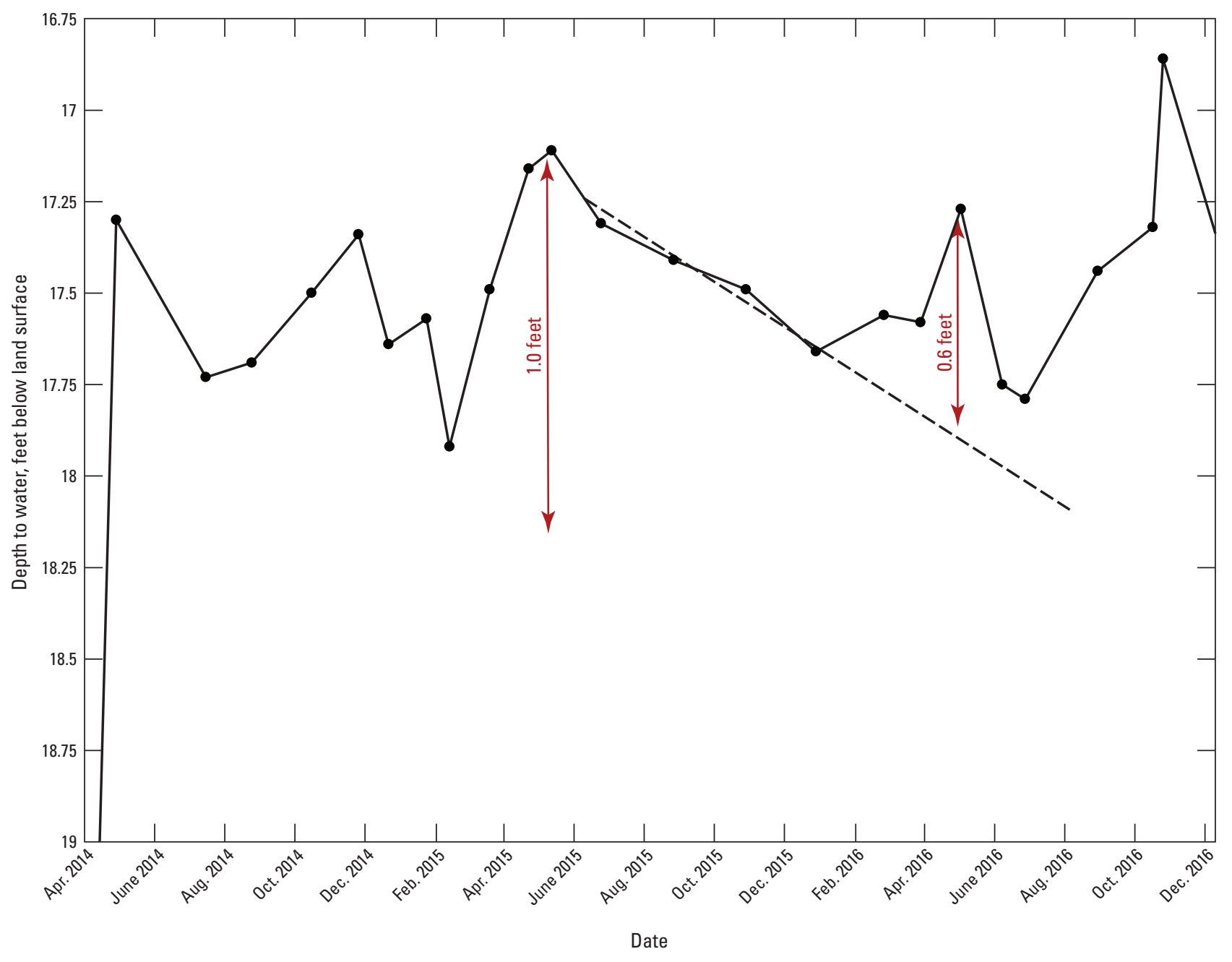

Figure 1.4. Peak water-table rise values (in red) used to determine estimated recharge for well 7 based on the graphical approach to the water-table fluctuation method. 


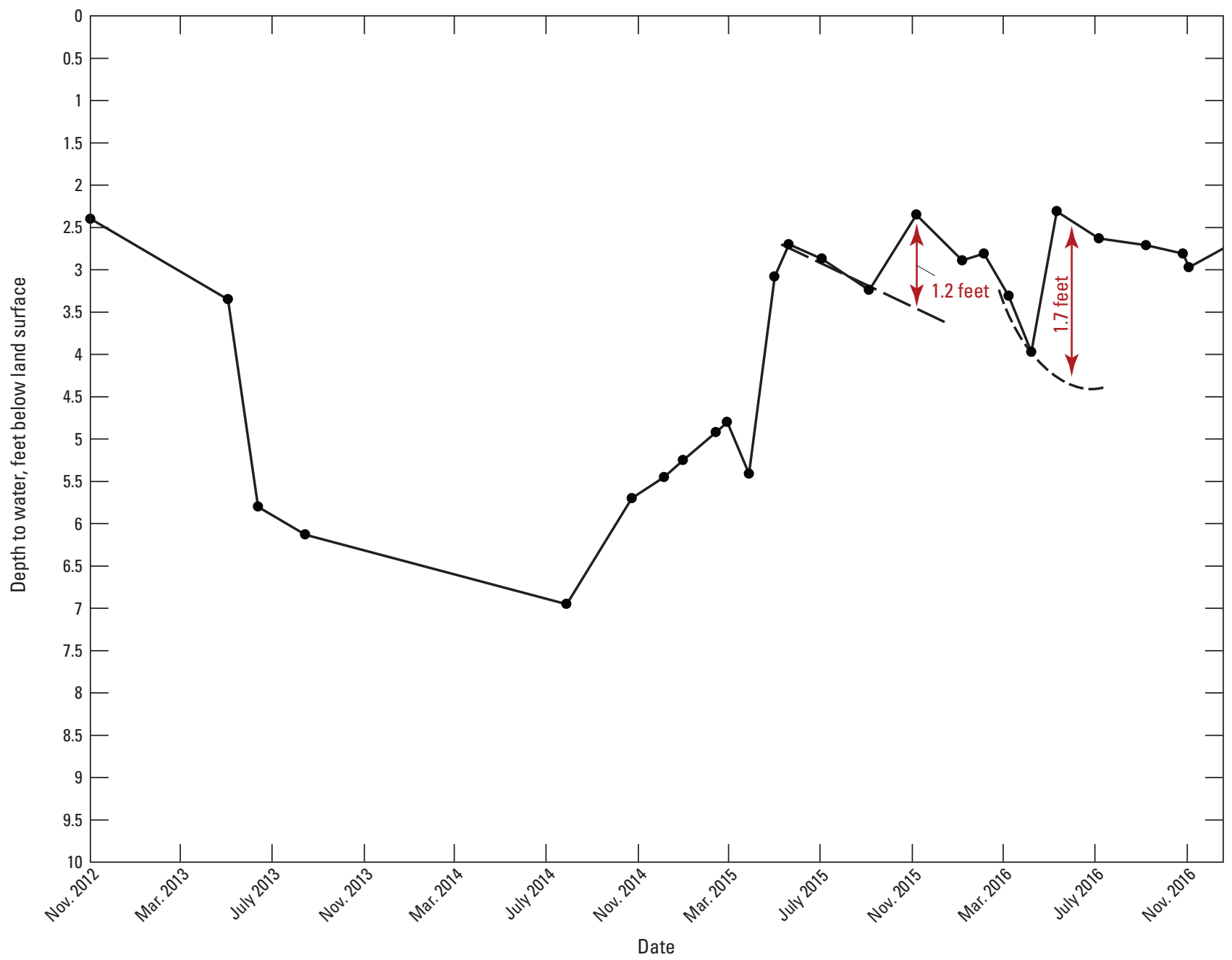

Figure 1.5. Peak water-table rise values (in red) used to determine estimated recharge for well 11 based on the graphical approach to the water-table fluctuation method. 


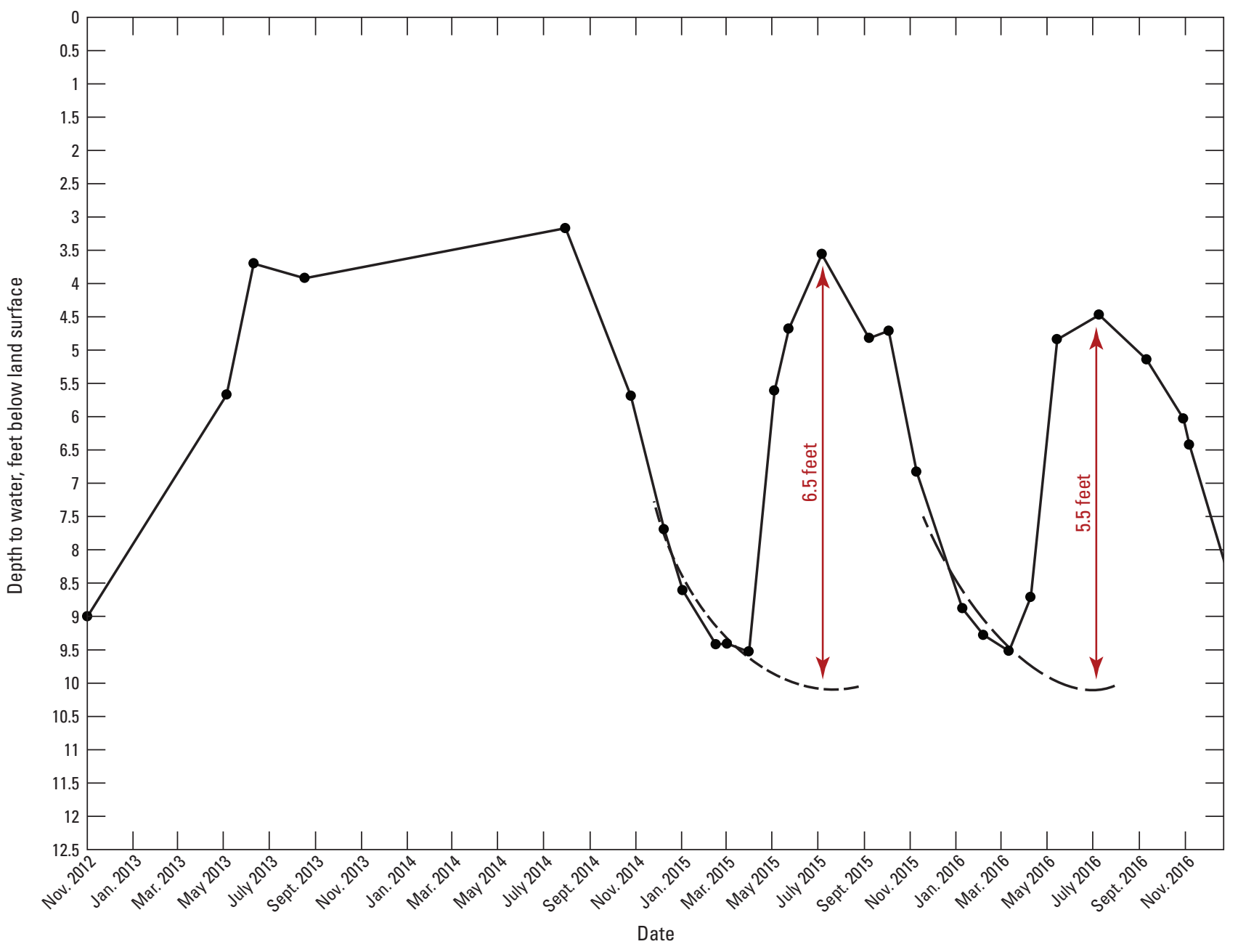

Figure 1.6. Peak water-table rise values (in red) used to determine estimated recharge for well 12 based on the graphical approach to the water-table fluctuation method. 


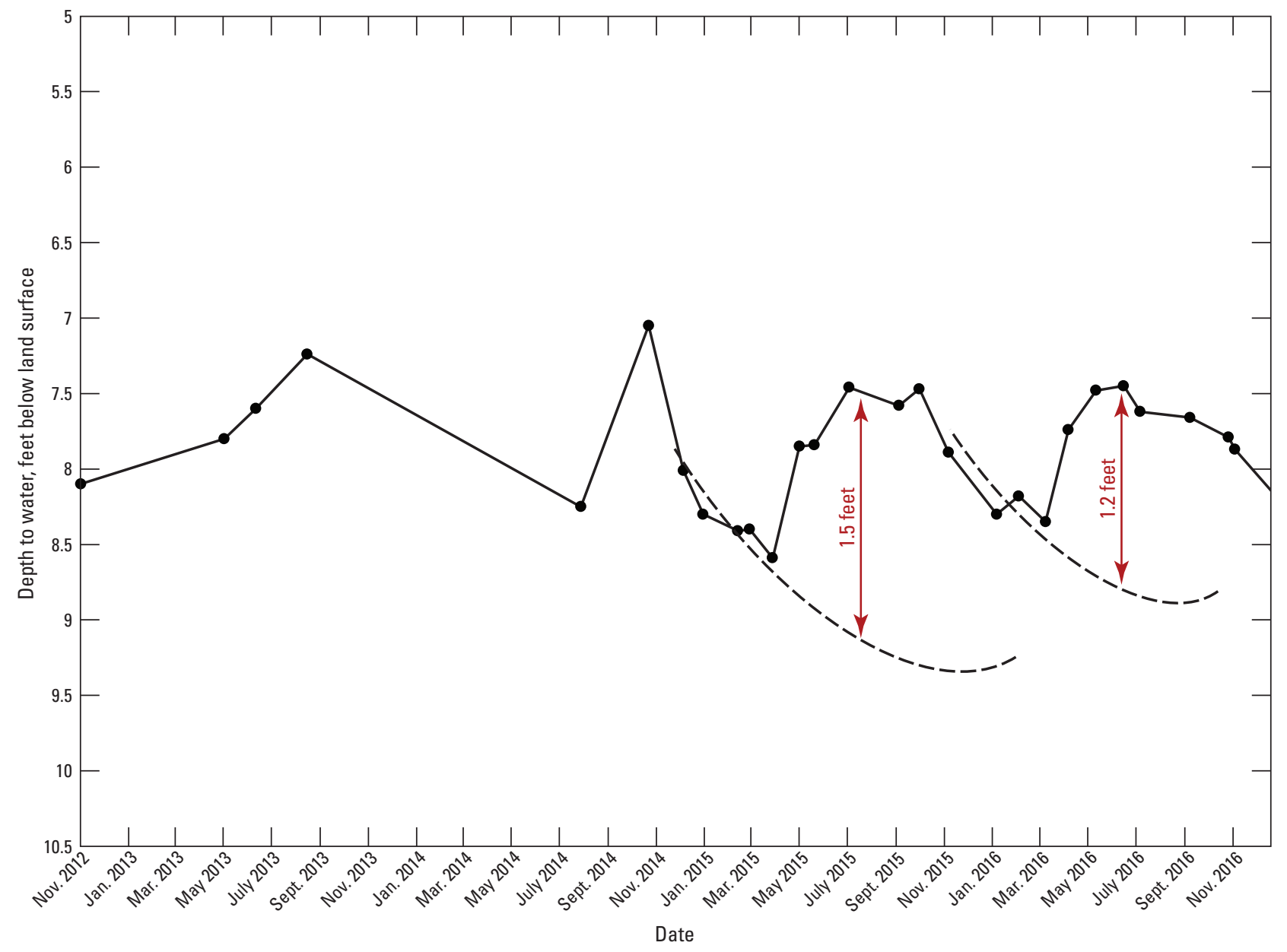

Figure 1.7. Peak water-table rise values (in red) used to determine estimated recharge for well 13 based on the graphical approach to the water-table fluctuation method. 


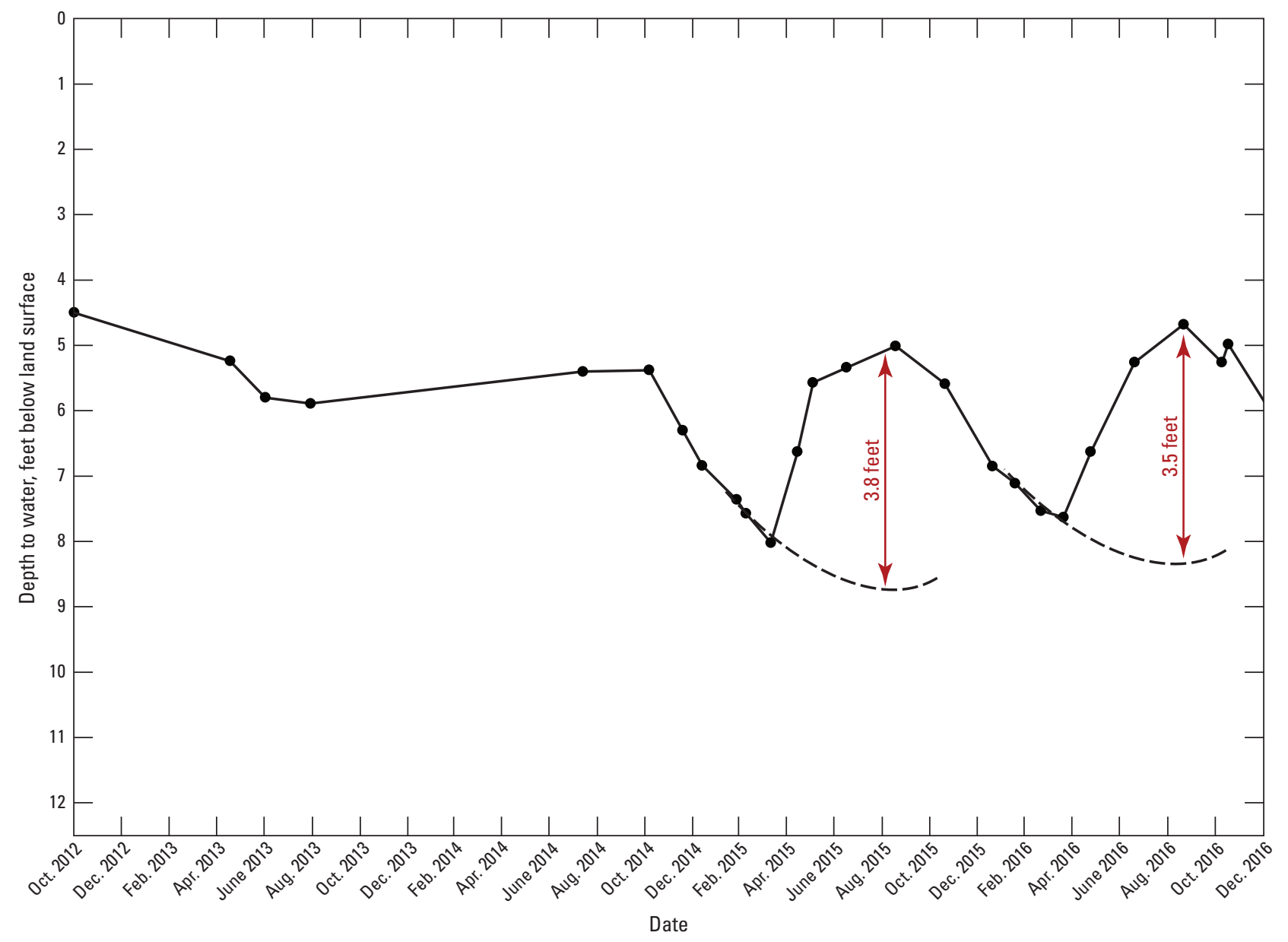

Figure 1.8. Peak water-table rise values (in red) used to determine estimated recharge for well 15 based on the graphical approach to the water-table fluctuation method. 


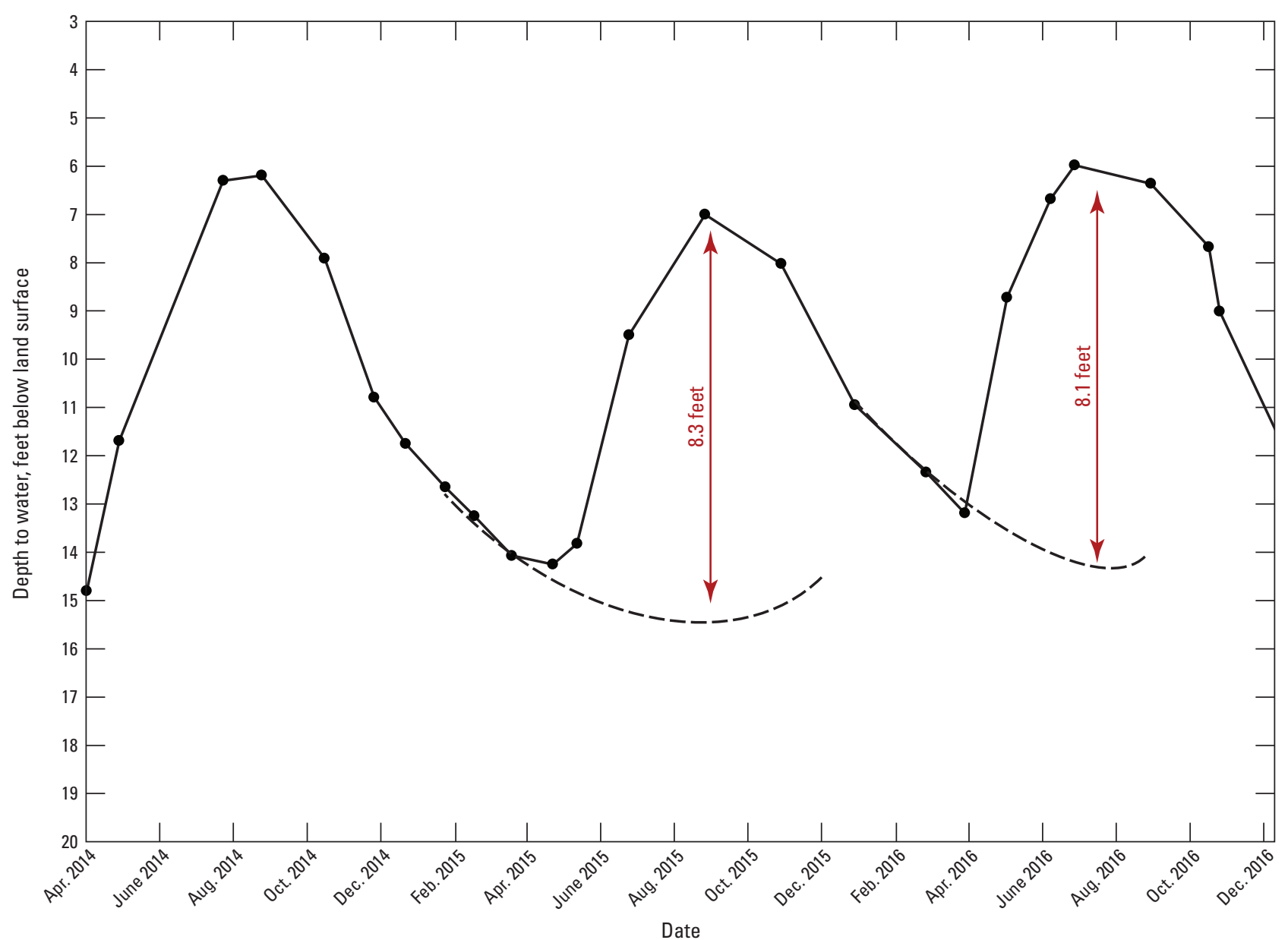

Figure 1.9. Peak water-table rise values (in red) used to determine estimated recharge for well 18 based on the graphical approach to the water-table fluctuation method. 


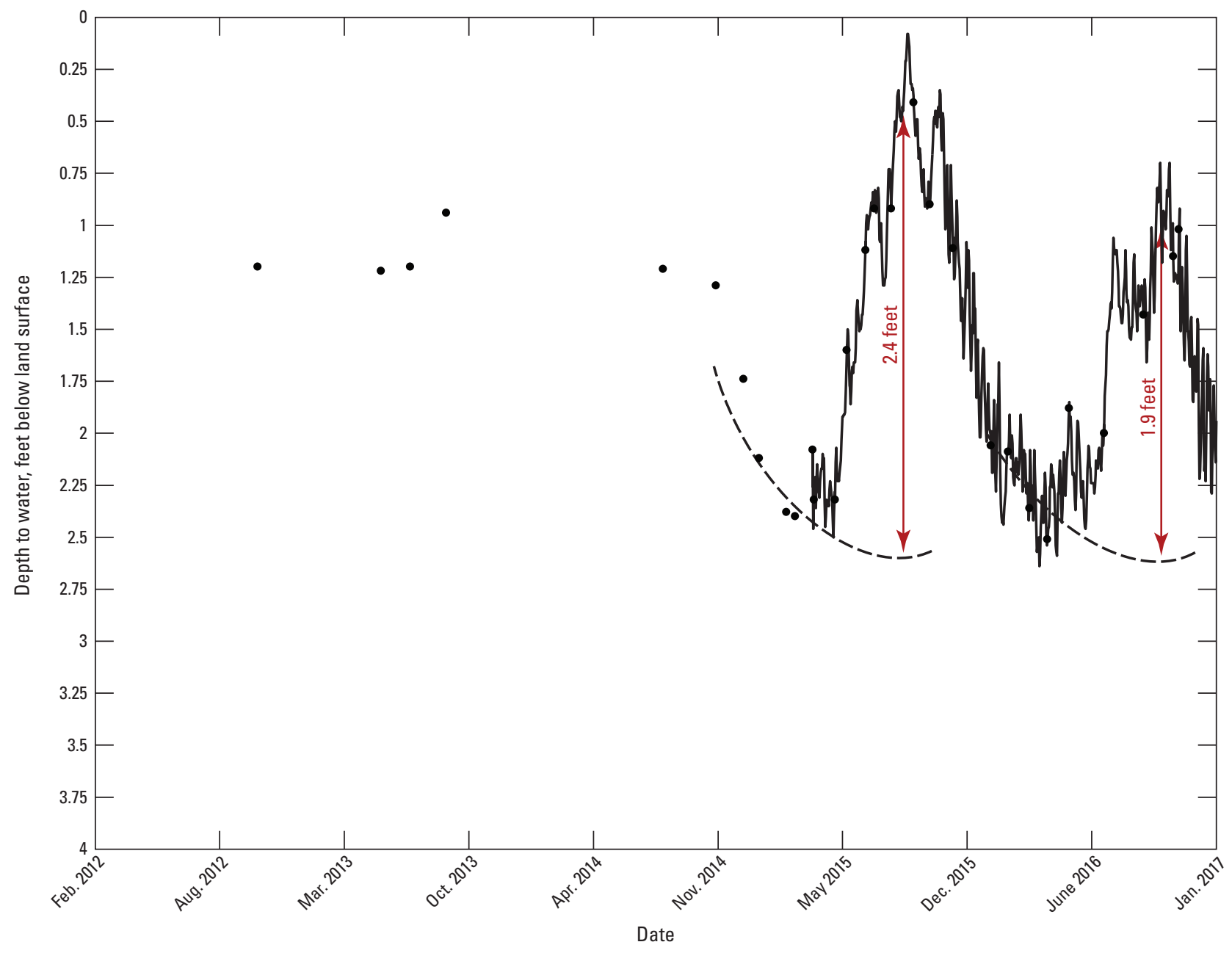

Figure 1.10. Peak water-table rise values (in red) used to determine estimated recharge for well 20 based on the graphical approach to the water-table fluctuation method. 


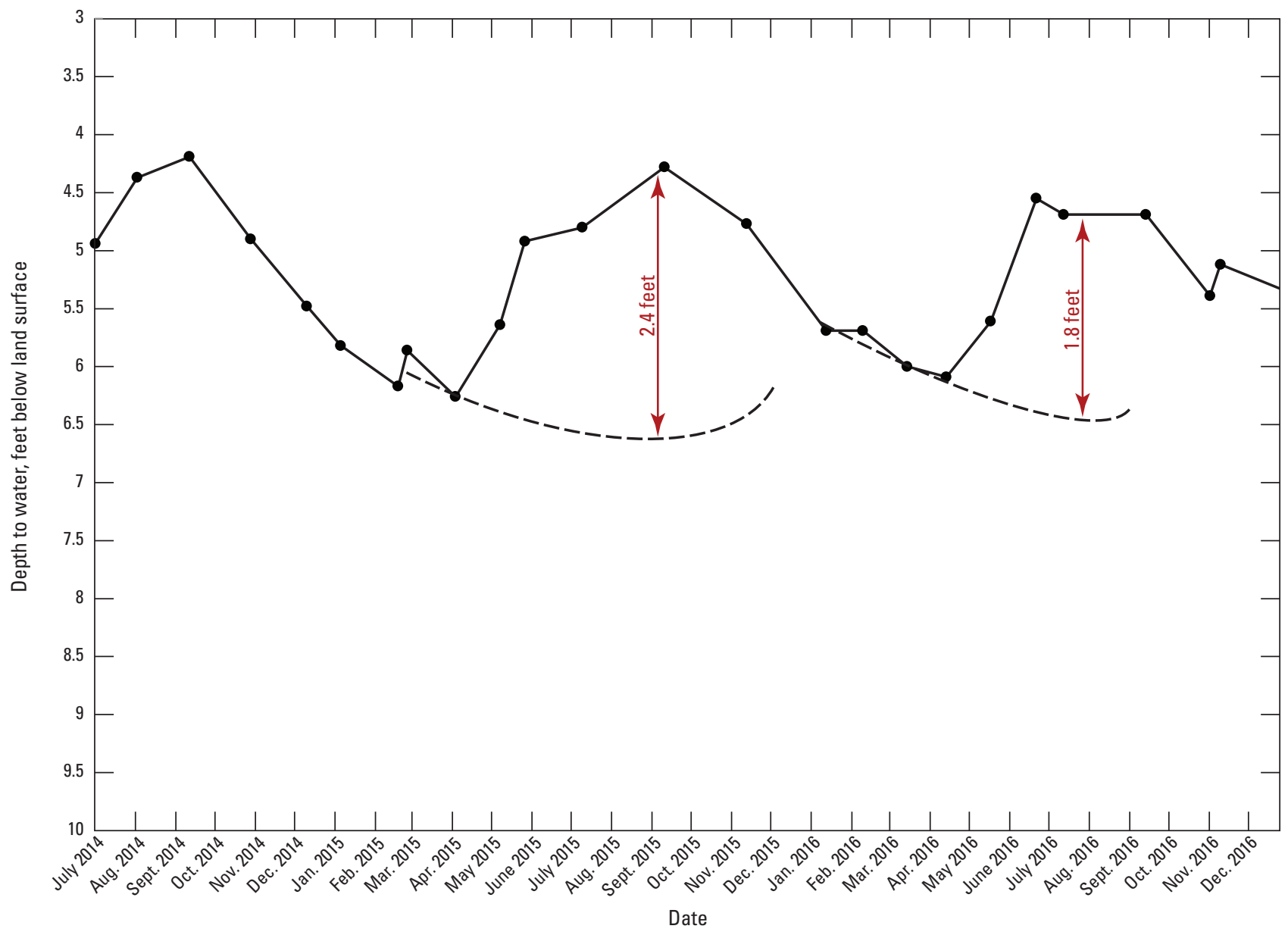

Figure 1.11. Peak water-table rise values (in red) used to determine estimated recharge for well 22 based on the graphical approach to the water-table fluctuation method. 


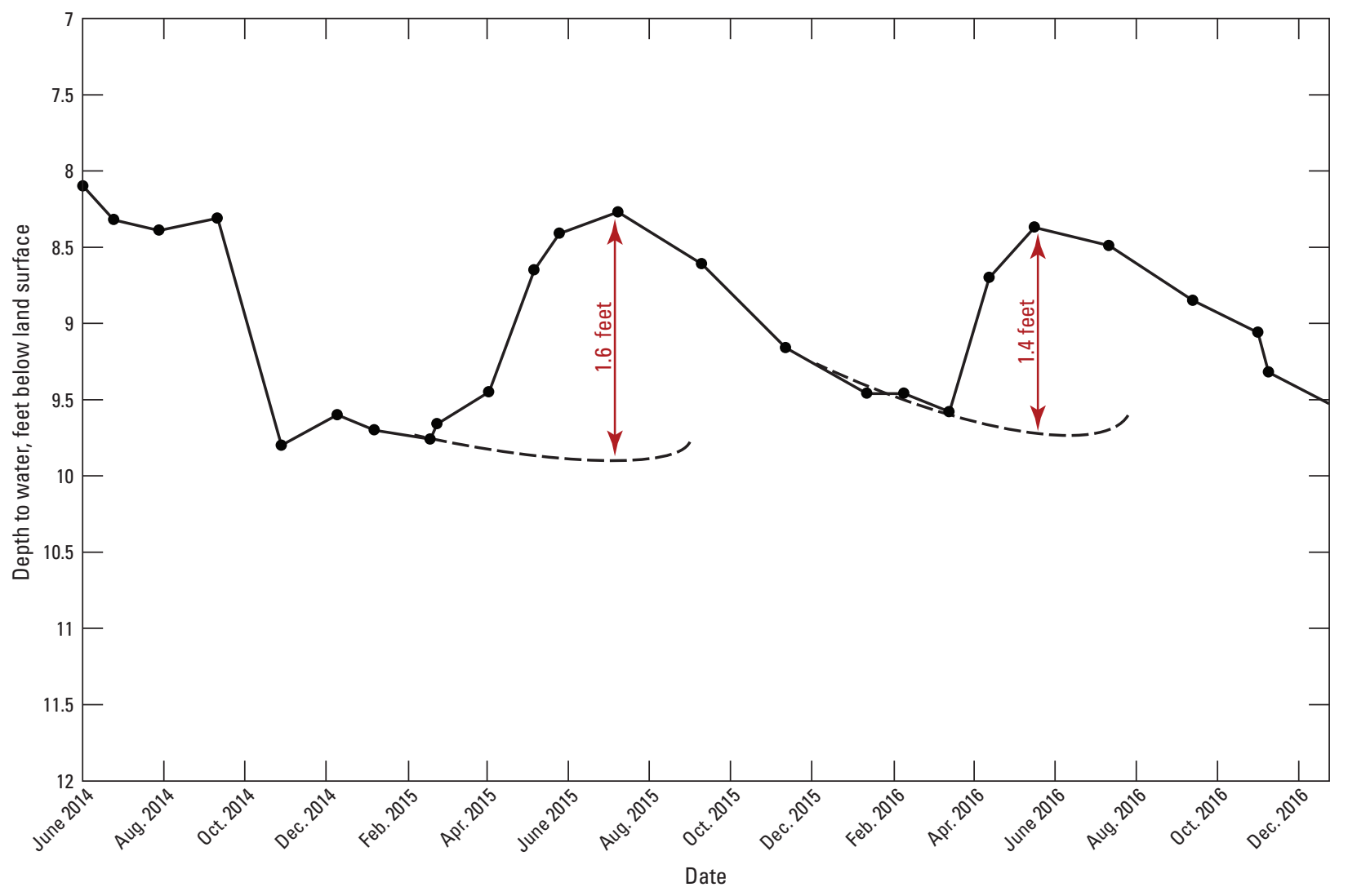

Figure 1.12. Peak water-table rise values (in red) used to determine estimated recharge for well 23 based on the graphical approach to the water-table fluctuation method. 


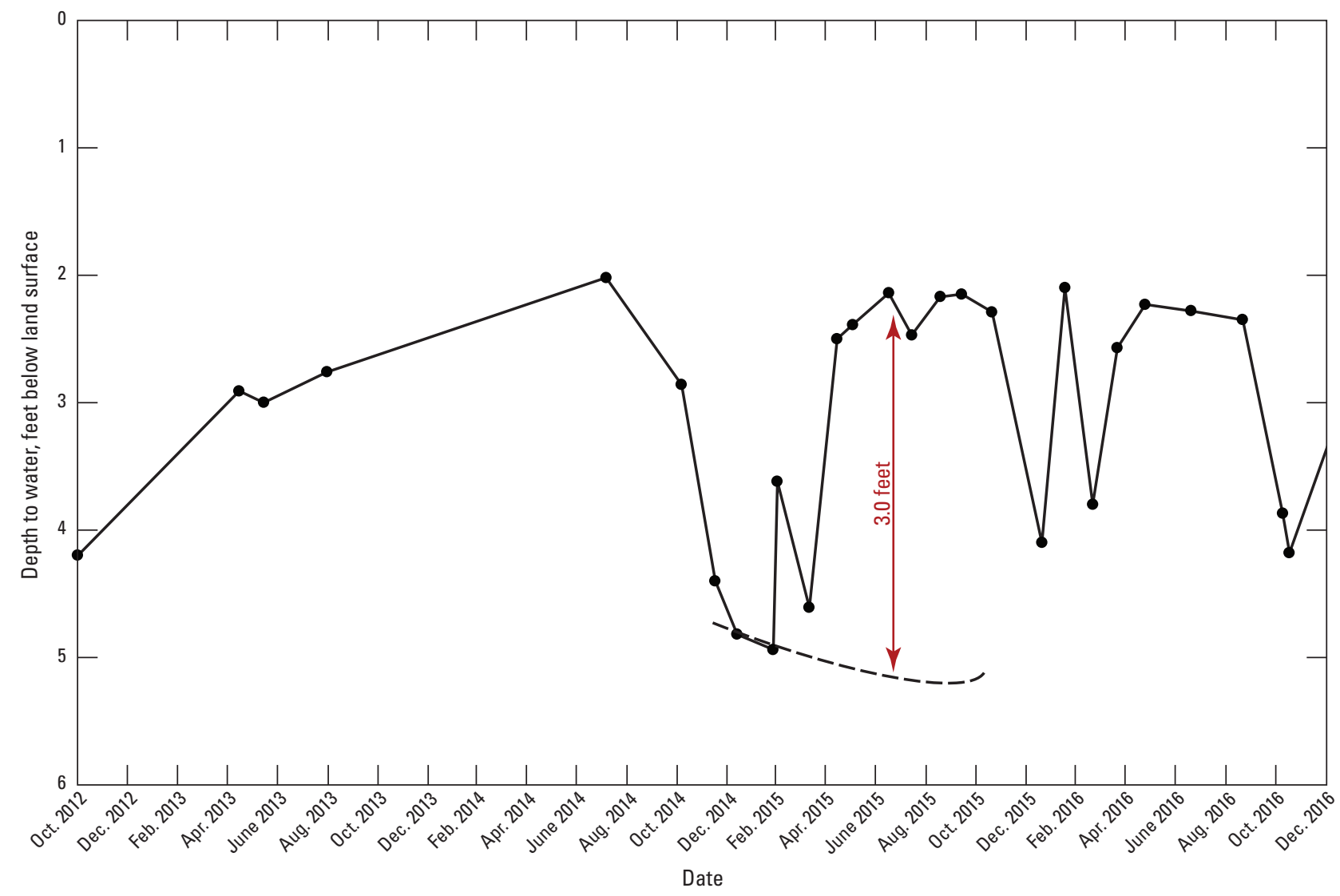

Figure 1.13. Peak water-table rise values (in red) used to determine estimated recharge for well 24 based on the graphical approach to the water-table fluctuation method. 


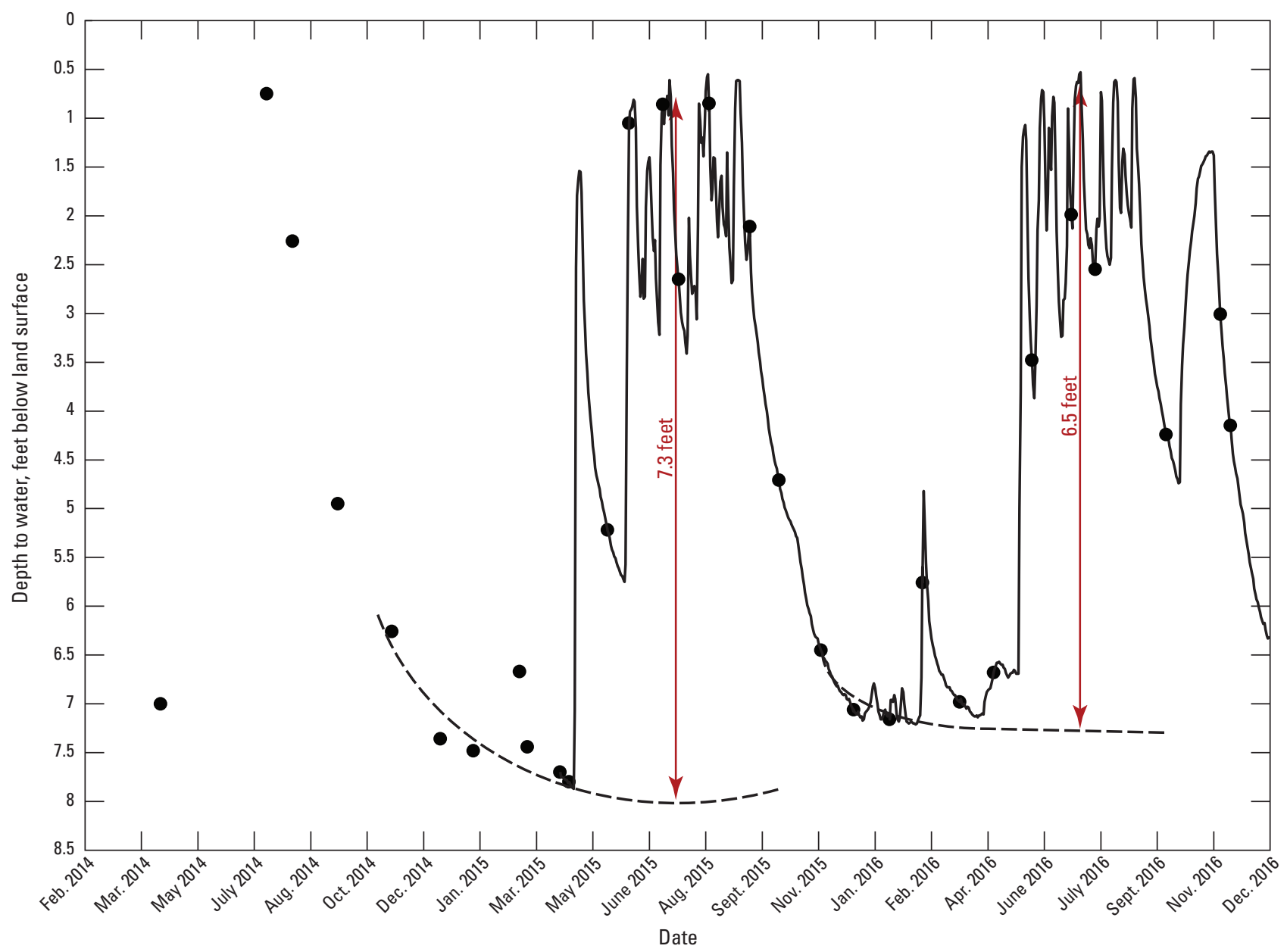

Figure 1.14. Peak water-table rise values (in red) used to determine estimated recharge for well 29 based on the graphical approach to the water-table fluctuation method. 


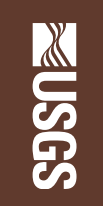

